\title{
Enabling Advanced Modeling and Simulations for Fuel-Flexible Combustors
}

\author{
Final Technical Report \\ Reporting Period: 3/15/2008 - 5/31/2010 \\ Professor Heinz Pitsch \\ Department of Mechanical Engineering \\ Stanford University \\ Phone: (650) 7361995 \\ Fax: (650) 7257834 \\ E-mail: h.pitsch@stanford.edu
}

October 1, 2010

Under Support of

Advance Coal Research Program at U.S. Colleges and Universities National Energy Technology Laboratory at U.S. Department of Energy

DoE Award No: DE-FG26-08NT43325 


\section{Disclaimer}

This report was prepared as an account of work sponsored by an agency of the United States Government. Neither the United States Government nor any agency thereof, nor any of their employees, makes any warranty, express or implied, or assumes any legal liability or responsibility for the accuracy, completeness, or usefulness of any information, apparatus, product, or process disclosed, or represents that its use would not infringe privately owned rights. Reference herein to any specific commercial product, process, or service by trade name, trademark, manufacturer, or otherwise does not necessarily constitute or imply its endorsement, recommendation, or favoring by the United States Government or any agency thereof. The views and opinions of authors expressed herein do not necessarily state or reflect those of the United States Government or any agency thereof. 


\section{Abstract}

The overall goal of the present project is to enable advanced modeling and simulations for the design and optimization of fuel-flexible turbine combustors. For this purpose we use a high-fidelity, extensively-tested large-eddy simulation (LES) code and state-of-the-art models for premixed/partially-premixed turbulent combustion developed in the PI's group. In the frame of the present project, these techniques are applied, assessed, and improved for hydrogen enriched premixed and partially premixed gas-turbine combustion. Our innovative approaches include a completely consistent description of flame propagation, a coupled progress variable/level set method to resolve the detailed flame structure, and incorporation of thermal-diffusion (non-unity Lewis number) effects. In addition, we have developed a general flamelet-type transformation holding in the limits of both non-premixed and premixed burning. As a result, a model for partially premixed combustion has been derived. The coupled progress variable/level method and the general flamelet tranformation were validated by LES of a lean-premixed low-swirl burner that has been studied experimentally at Lawrence Berkeley National Laboratory. The model is extended to include the non-unity Lewis number effects, which play a critical role in fuel-flexible combustor with high hydrogen content fuel. More specifically, a two-scalar model for lean hydrogen and hydrogen-enriched combustion is developed and validated against experimental and direct numerical simulation (DNS) data. Results are presented to emphasize the importance of non-unity Lewis number effects in the lean-premixed low-swirl burner of interest in this project. The proposed model gives improved results, which shows that the inclusion of the non-unity Lewis number effects is essential for accurate prediction of the lean-premixed low-swirl flame. 


\section{Contents}

1 Executive Summary . . . . . . . . . . . . . . . . . . . . 4

2 Turbulent Combustion Model . . . . . . . . . . . . . . . . 5

2.1 LES of Premixed Turbulent Combustion: Flame Surface Filtering and Dynamic Flame Speed Model . . . . . . . . . . . . . . . 5

2.2 A General Flamelet Transformation for Premixed and Non-Premixed Combustion: Combustion Regime Index _ . . . . . . . . . . . 8

2.3 Partially Premixed Combustion . . . . . . . . . . . . . . . . . 10

3 Governing Equations and Numerical Method . . . . . . . . . . . . . . . 11

4 Validation: Premixed Swirl Flames and Partially Premixed Combustion . . . 12

4.1 Turbulent Swirling Flames . . . . . . . . . . . . . . . . . . . . . . . 12

4.2 Lean-Premixed Low-Swirl Hydrogen Flames . . . . . . . . . . . . . 14

5 Simulation of a Low Swirl Combustor . . . . . . . . . . . . . . . . 20

5.1 Cold Flow Simulation . . . . . . . . . . . . . . . . . . . . 20

5.2 Methane Flame . . . . . . . . . . . . . . . . . . . . . 23

5.3 Hydrogen Flame . . . . . . . . . . . . . . . . . . . . . . 26

5.4 Flash-Back and Blow-Out . . . . . . . . . . . . . . . . . 27

6 Model Extension for Hydrogen Combustion . . . . . . . . . . . . . . . . . . 27

6.1 Mixture Fraction/Progress Variable Approach for Lean Hydrogen Premixed Combustion . . . . . . . . . . . . . . . . . 30

6.2 Hydrogen/Progress Variable Approach for Lean Hydrogen Premixed Combustion . . . . . . . . . . . . . . . 36

7 Validation Using Direct Numerical Simulation Data for Lean Hydrogen Combustion . . . . . . . . . . . . . . . . . . . . . 36

8 Application to a Low-Swirl Hydrogen Flame . . . . . . . . . . . . . . . . 44

8.1 Large Eddy Simulation Using the Mixture-Fraction/Progress-Variable Approach . . . . . . . . . . . . . . . . . . . 44

8.2 Large Eddy Simulation Using the Hydrogen/Progress-Variable Approach 46 


\section{Executive Summary}

Gas turbines are known as the main source of electrical energy in the modern world. Permanent increase in the oil and gas price, diminishing resources and global climate change demand clean and efficient usage of coal as well as the utilization of renewable resources and opportunity feed-stocks such as petroleum coke and biomass. These needs call for the development of advanced next-generation turbine technologies. Such a goal can be met by the assistance of advanced simulations that are validated by reliable comprehensive experimental data.

The overall goal of the present project is to enable advanced modeling and simulations for the design and optimization of fuel-flexible turbine combustors. For this purpose we use a high-fidelity, extensively-tested large-eddy simulation (LES) code and state-of-theart models for partially premixed turbulent combustion. The LES code solves for the filtered Navier-Stokes equations in the low Mach number limit using the semi-implicit finitedifference method, and it can have any high order of numerical accuracy in space discretization, with high computational efficiency [1]. The solver has been extensively used to simulate combustion processes in several applications, for example, in combustors and industrial furnaces. Numerous physico-chemical and numerical models describing turbulent combustion have been proposed in previous work. In the recent past, we have developed several new modeling techniques related to combustion LES. In the frame of the present project, these techniques are applied, assessed, and improved for hydrogen enriched premixed and partially premixed gas-turbine combustion.

Our innovative approaches include a completely consistent description of flame propagation, a coupled progress variable/level set method to resolve the detailed flame structure, and incorporation of thermal-diffusion (non-unity Lewis number) effects. In addition, we have developed a general flamelet-type transformation holding in the limits of both non-premixed and premixed burning $[2,3]$. As a result, a model for partially premixed combustion has been derived. The coupled progress variable/level method and the general flamelet tranformation were validated by LES of a lean-premixed low-swirl burner that has been studied experimentally at Lawrence Berkeley National Laboratory.

In this report, we summarize the accomplishments of the project. The activity in the frame of the project includes three main phases. At Phase I, we perform LES of a cold flow, study inlet boundary conditions and test the LES solver by available experimental data and DNS results. At Phase II, we investigate reacting flows. First, we generate an appropriate chemical table containing a set of flamelet parameters and used by the solver. The LES solver is validated by combustion available experiments/simulations. Then we perform LES of a modeled combustor and test the simulations by turbine combustors from NETL, Sandia CRF, and Berkeley Lab. Flame blowout and flashback are also considered. The model is validated and the area of further improvement is identified. At Phase III, the model is extended to include the non-unity Lewis number effects, which play a critical role in fuelflexible combustor with high hydrogen content fuel. A two-scalar model for lean hydrogen and hydrogen-enriched combustion is developed and validated. The main physical phenomena 
of interest are the effects of non-unity Lewis numbers and diffusive-thermal instability on turbulent flame propagation. Results are presented to emphasize the importance of these effects in the lean-premixed low-swirl burner of interest in this project. First, the two-scalar model is validated using direct numerical simulation (DNS) data for a freely propagating lean hydrogen premixed flame. The two-scalar model is shown to capture the dependence of the flame speed on the flame curvature reasonably well. Second, the two-scalar model has been applied to a lean hydrogen premixed low-swirl injector, for which existing combustion models fail. The hydrogen content and progess-variable (or mixture fraction and progress variable) are chosen as flamelet parameters to describe the local flame structure. The results show that the inclusion of the non-unity Lewis number effects is essential for accurate prediction of the lean-premixed low-swirl flame. However, both of LES and DNS studies suggest that further improvement of the model is warranted.

\section{Turbulent Combustion Model}

As we stressed in Sec. 1, the general objective of the present project is to enable advanced simulations and modeling for the design and optimization of fuel-flexible turbine combustors. This goal is being achieved by using high-fidelity extensively-tested LES and state-of-the-art models for turbulent combustion.

In LES, turbulence is properly resolved and directly computed on large-scales, while small-scale turbulent structures are left unresolved and are modeled [4]. Various methods have been suggested in the literature regarding the problem of subgrid transition. Two of the most widely used are the flame thickening [5] and the level set (or G-equation) approach $[6,7]$. The thickened flame model is based on an artificial increase in molecular diffusivities, with modified reaction source terms in order to keep the planar flame speed $S_{L}$ constant. As a result, the local reaction zones become boarded and can be resolved on LES meshes. In level set methods, a flame front is treated as an isocontour of a field variable that is explicitly tracked. At the relevant isocontours, the variables are governed by equations describing how the fronts propagate. Far away from the isocontours, smooth gradients are prescribed for the field variables to ensure numerical resolution of the flame front dynamics.

There is a great variety of LES codes of different levels of sophistication. In the present project, we are using our in-house semi-implicit finite-difference LES solver [1]. As we note above, the code has been extensively used to simulate combustion processes in various applications. Here we will start with a description of premixed flamelets; then non-premixed turbulent burning will be considered.

\subsection{LES of Premixed Turbulent Combustion: Flame Surface Fil- tering and Dynamic Flame Speed Model}

A premixed flame front can be defined as an isocontour of a generic progress variable $c$, which might be, say, a non-dimensionalized temperature, enthalpy, etc. The governing equation 
for the progress variable is

$$
\frac{\partial c}{\partial t}+u_{j} \frac{\partial c}{\partial x_{j}}=\frac{1}{\rho} \frac{\partial}{\partial x_{j}}\left(\rho \mathcal{D} \frac{\partial c}{\partial x_{j}}\right)+\frac{1}{\rho} \dot{\omega}_{R}
$$

where $u_{j}$ is the local flow velocity in the $j$ th direction, $\rho$ is the fluid density, $\mathcal{D}$ is the diffusivity of the variable $c$ and $\dot{\omega}_{R}$ is a source term that describes the effects of chemical reactions. For an arbitrary isosurface $c=c_{0}$, Eq. (1) may be rewritten as

$$
\begin{aligned}
& \frac{\partial\left[H\left(c-c_{0}\right)\right]}{\partial t}+u_{j} \frac{\partial\left[H\left(c-c_{0}\right)\right]}{\partial x_{j}}= \\
& \mathcal{D} \kappa\left|\nabla\left[H\left(c-c_{0}\right)\right]\right|+\delta\left(c-c_{0}\right) \frac{1}{\rho}\left[n_{j} \frac{\partial}{\partial x_{j}}(\rho \mathcal{D}|\nabla c|)+\dot{\omega}_{R}\right],
\end{aligned}
$$

where $\kappa$ is the front curvature, $n_{j}$ is the front normal vector and $H$ represents a Heaviside function, or equivalently an error function of small but finite width, that is centered on isocontour $c=c_{0}$; see [2] for the details. When the chosen progress variable isocontour is associated with a location in the flame front, Eq. (2) can be treated as the equation governing the behavior of a Heaviside function that tracks the flame front. For notational convenience, we introduce a level set variable $\mathcal{G}$

$$
\mathcal{G}\left(x_{j}, t\right)=H\left(c\left(x_{j}, t\right)-c_{0}\right) .
$$

Then Eq. (2) takes the form

$$
\frac{\partial \mathcal{G}}{\partial t}+u_{j} \frac{\partial \mathcal{G}}{\partial x_{j}}=\mathcal{D} \kappa|\nabla \mathcal{G}|+S_{L, c_{0}}|\nabla \mathcal{G}|
$$

where the value

$$
S_{L, c_{0}}=\left[\frac{1}{|\nabla c| \rho}\left(n_{j} \frac{\partial}{\partial x_{j}}(\rho \mathcal{D}|\nabla c|)+\dot{\omega}_{R}\right)\right]_{c=c_{0}}
$$

describes the propagation velocity of isosurface $c=c_{0}$. In the absence of curvature and strain effects, it is reduced to the unstretched laminar flame speed $S_{L}$. Equation (4) is meaningfully defined in the whole of physical space and could therefore be filtered in line with standard LES filtering techniques as [2]

$$
\frac{\partial \overline{\mathcal{G}}}{\partial t}+\overline{u_{j} \frac{\partial \mathcal{G}}{\partial x_{j}}}=(\overline{\mathcal{D} \kappa})_{T}|\nabla \overline{\mathcal{G}}|+\bar{S}_{T, c_{0}}|\nabla \overline{\mathcal{G}}|
$$

where $\bar{S}_{T, c_{0}}$ and $(\overline{\mathcal{D} \kappa})_{T}$ are the filtered burning velocity and the filtered curvature, respectively. They are determined as

$$
\bar{S}_{T, c_{0}}|\nabla \overline{\mathcal{G}}|=\overline{S_{L, c_{0}}|\nabla \mathcal{G}|}, \quad(\overline{\mathcal{D} \kappa})_{T}|\nabla \overline{\mathcal{G}}|=\overline{\mathcal{D} \kappa|\nabla \mathcal{G}|} .
$$

Equation (6) describes properly dynamics of a turbulent flame propagation speed. However, the unclosed convective term in Eq. (6) cannot be dealt with in LES. For this reason, an 
additional model is introduced to separate the resolved and unresolved components. As described in [2], the unresolved components are actually negligible. In addition, the effect of the density change through the flame was removed from the propagation speed term. Then the level set equation takes the final form

$$
\frac{\partial \overline{\mathcal{G}}}{\partial t}+\widetilde{u}_{j} \frac{\partial \overline{\mathcal{G}}}{\partial x_{j}}=\frac{\rho_{u}}{\bar{\rho}}\left[(\overline{\mathcal{D} \kappa})_{T, u}+\bar{S}_{T, u}\right]|\nabla \overline{\mathcal{G}}| .
$$

Now let us consider a dynamic procedure for computing the coefficient that appears in models for $\bar{S}_{T, u}$. This procedure requires an LES test filter denoted hereafter by the "hat" operator. The "hat" operator represents the same kind of filtering process as the "overline" one does, but uses a larger filter length scale. Equation (8) can be filtered either in a serial process (where these filters are applied simultaneously), or in a sequential process (where these filters are applied in different steps). These two options yield the equations

$$
\begin{aligned}
& \frac{\partial \overline{\mathcal{G}}}{\partial t}+\widetilde{u}_{j} \frac{\partial \overline{\mathcal{G}}}{\partial x_{j}}+=\frac{\rho_{u}}{\bar{\rho}}(\overline{\mathcal{D} \kappa})_{T}|\nabla \overline{\mathcal{G}}|+\frac{\rho_{u}}{\bar{\rho}} \bar{S}_{T, u}|\nabla \overline{\mathcal{G}}|, \\
& \frac{\partial \overline{\mathcal{G}}}{\partial t}+\widetilde{u}_{j} \frac{\partial \overline{\mathcal{G}}}{\partial x_{j}}+=\frac{\rho_{u}}{\bar{\rho}}(\overline{\mathcal{D} \kappa})_{T}|\nabla \overline{\mathcal{G}}|+\frac{\rho_{u}}{\bar{\rho}} \bar{S}_{T, u}|\nabla \overline{\mathcal{G}}|,
\end{aligned}
$$

respectively. Assume that the filters commute with temporal derivatives. Then the subtraction of Equations (9) and (10) gives

$$
\widetilde{u}_{j} \frac{\partial \overline{\mathcal{G}}}{\partial x_{j}}-\left(\frac{\rho_{u}}{\bar{\rho}}(\overline{\mathcal{D} \kappa})_{T}+\frac{\rho_{u}}{\bar{\rho}} \bar{S}_{T, u}\right)|\nabla \overline{\mathcal{G}}|=\widetilde{u}_{j} \frac{\partial \overline{\mathcal{G}}}{\partial x_{j}}-\left(\frac{\rho_{u}}{\bar{\rho}}(\overline{\mathcal{D} \kappa})_{T}+\frac{\rho_{u}}{\bar{\rho}} \bar{S}_{T, u}\right)|\nabla \overline{\mathcal{G}}| .
$$

Equation (11) does not contain temporal derivatives; it holds at all times.

This model is not yet applicable in an LES because the $\mathcal{G}$ variable is never actually solved for. It is in fact computationally intractable, since jumps in $\overline{\mathcal{G}}$ occur over single filter widths. Instead, the terms in which this variable appears must be modeled using the level set representation of the flame front. The developed model, which holds in both the fully resolved and fully unresolved limits, is

$$
|\nabla \overline{\mathcal{G}}| \approx \bar{A}_{\Delta} / V_{\Delta}
$$

where $\bar{A}_{\Delta} / V_{\Delta}$ represents the area density of the filtered flame front, as described by the level set evaluated at the LES filter level. It follows from this that the convective terms in Eq. (11) can be modeled as

$$
\widetilde{u}_{j} \frac{\partial \overline{\mathcal{G}}}{\partial x_{j}} \approx \widetilde{u}_{j} \bar{n}_{j} \frac{\bar{A}_{\Delta}}{V_{\Delta}}
$$


Then the dynamics equation takes the final form

$$
\widetilde{u}_{j} \bar{n}_{j} \frac{\bar{A}_{\Delta}}{V_{\Delta}}-\left(\frac{\rho_{u}}{\bar{\rho}}(\overline{\mathcal{D} \kappa})_{T}+\frac{\rho_{u}}{\bar{\rho}} \bar{S}_{T, u}\right) \frac{\bar{A}_{\Delta}}{V_{\Delta}}=\widetilde{u}_{j} \bar{n}_{j} \frac{\bar{A}_{\Delta \Delta}}{V_{\Delta \Delta}}-\left(\frac{\rho_{u}}{\bar{\rho}}(\overline{\mathcal{D} \kappa})_{T}+\frac{\rho_{u}}{\bar{\rho}} \bar{S}_{T, u}\right) \frac{\bar{A}_{\Delta \Delta}}{V_{\Delta \Delta}}
$$

Equation (14) is a dynamic identity that can be solved to locally calculate a flame speed model parameter. To apply Eq. (14) to the determination of an unknown constant, the actual burning velocity and diffusive propagation models must be selected. The filtered burning velocity may be described by Peters' propagation speed model $[7,8]$

$$
\frac{\bar{S}_{T, u}-S_{L, u}}{u^{\prime}}=-\gamma \mathrm{Da}+\sqrt{(\gamma \mathrm{Da})^{2}+\gamma \alpha \mathrm{Da}}
$$

where $\alpha$ and $\gamma$ are model constants, and $u^{\prime}$ and $D a$ are the velocity fluctuation and Damköhler number evaluated at the filter level $\Delta$. When the curvature models and Eq. (15) for the flame speed are substituted into Eq. (14), all the terms in the result except $\alpha$ are known. Solving for $\alpha$ and then returning that value to the flame speed model gives filter independent front locations.

As a result, a surface-filtering procedure consistent with standard LES-filtering has been derived [2]. The method has been extensively tested in the context of DNS and applied in the context of LES [2]. The approach uses only information that comes directly from the flame front location. This is important for two reasons: (i) it guarantees that the model can be consistently applied when level set methods (where arbitrary constraints can be imposed on field variables away from fronts) are used to track the flame; (ii) it forces the model to recognize that the physics governing flame propagation of a flame are valid only locally at the front.

\subsection{A General Flamelet Transformation for Premixed and Non- Premixed Combustion: Combustion Regime Index}

The technique presented in the previous subsection describes fully premixed combustion. However, one often deals with non-premixed or partially premixed burning in laboratory and industrial setups. Thus a general model that distinguishes between these regimes is required. For this purpose, a general flamelet transformation that holds in the limits of both premixed and non-premixed combustion has been recently developed [3]. The basic idea of the method is to use two statistically independent parameters such as the reaction progress variable and the mixture fraction.

Assume that a set of scalar transport equations is written in a standard "physical" space $(\mathbf{x}, t)$. The steady flamelet equations describing the asymptotic limit of non-premixed combustion are typically derived by reorienting the coordinate system $(\mathbf{x}, t)$ into a basis in which one direction is parallel to the local gradient of mixture fraction, $\nabla Z$. Then we assume that changes in the scalar field along directions, not parallel to the gradient of the 
mixture fraction, are negligible in comparison with the changes that occur in the direction of the gradient [8]. In a similar way, another set of equations can be derived to describe the asymptotic limit of premixed combustion. An indicator of the regime of burning can be developed by combining these ideas into a more general transformation that concurrently demonstrates how the chemical source term is balanced in both the premixed and nonpremixed limits. Any of such transformations needs to retain the coordinate direction of the mixture fraction $Z$, since it describes the non-premixed limit. From the first point of view, the progress variable $c$ might be a second coordinate direction describing the premixed limit. However, $Z$ and $c$ (as they are typically defined) are not statistically independent, while statistical independence is manifested most clearly in a non-premixed flamelet. If the eventually developed transformation is to reduce to the steady flamelet equations in the non-premixed limit, then the second transformation coordinate has to disappear in that limit. The progress variable, however, changes with $Z$ in a non-premixed flamelet, and this condition is not satisfied. This implies that partial derivatives of a scalar cannot be consistently taken with respect to $(Z, c)$ space.

To indicate the regime of burning, non-premixed flamelets are indexed, and the value of the index of each flamelet is treated as a variable. Basically, there is a variety of choices for the flamelet index. As an option, one can choose [9]

$$
\Lambda=c\left(Z_{\mathrm{st}}, T_{Z_{\mathrm{st}}}^{*}\right)
$$

which is the value of the progress variable that occurs on a given flamelet $\left(T_{Z_{\mathrm{st}}}=T_{Z_{\mathrm{st}}}^{*}\right)$ and at a given mixture fraction $\left(Z=Z_{\text {st }}\right)$. Obviously, the dependence versus $\Lambda$ disappears in the limit of non-premixed combustion, since there is a unique value of $\Lambda$ associated with each flamelet. In addition, taking partial derivatives of a scalar with respect to space $(Z, \Lambda)$ is consistent, since $\Lambda$ and $Z$ are statistically independent.

The transformation technique used to develop the flamelet equations can be also used to transform a scalar equation from space $(\mathbf{x}, t)$ into space $(Z(\mathbf{x}, t), \Lambda(\mathbf{x}, t), e(\mathbf{x}, t), \tau)$. This is accomplished through usage of the rules

$$
\partial_{t} \rightarrow \partial_{\tau}+\left(\partial_{t} Z\right) \partial_{Z}+\left(\partial_{t} \Lambda\right) \partial_{\Lambda}, \quad \nabla \rightarrow(\nabla Z) \partial_{Z}+(\nabla \Lambda) \partial_{\Lambda}+\epsilon(\nabla e) \partial_{e},
$$

where $\partial_{t} \tau=1$ and $\partial_{t} e=0$. The parameter $\epsilon$ is introduced in order to describe the assumption that variations in the scalar along the third direction, $e$, are small. Applying these rules and neglecting the unsteady effects and the terms of order $\epsilon$, we find (see [3] for the details)

$$
\left(\partial_{\Lambda} c\left[\rho_{u} S_{L, u}|\nabla \Lambda|-\nabla \cdot(\rho \mathcal{D} \nabla \Lambda)\right]\right)_{p}+\left(-\rho \frac{\chi_{Z}}{2} \partial_{Z}^{2} c\right)_{n p}=\dot{\omega}_{c}
$$

Here the groups in brackets labeled "p" and "np" describe the premixed and non-premixed limits, respectively. To distinguish between the regimes, we simply evaluate the expressions in both groups. If the sum of the terms in group "p" is larger, then the premixed mode of combustion more appropriately describes the local process. In contrast, if the sum of the terms in group "np" is dominant, then the process is appropriately described by the mode of non-premixed burning. 
In the majority of situations, this model will require the use of tabulated chemistry. For example, the $\partial_{\Lambda} c, S_{L, u}$ and $\partial_{Z}^{2} c$ terms may all need to be evaluated using pre-computed tables. The functional form of the tabulated chemistry is not particularly relevant to the model, as long as it can be shown to be consistent with the parameterizations $\phi_{i}=\phi_{i}(Z, \Lambda)$. The flamelet progress variable would, for example, offer an appropriate context for tabulating chemistry when the functional dependency $\Lambda=\mathcal{G}(Z, c)$ is well defined. Evaluating the relative importance of terms in "p" and "np" can in general be determined by evaluating a time scale ratio

$$
\Theta=\frac{\partial_{\Lambda} c\left[\rho_{u} S_{L, u}|\nabla \Lambda|-\nabla \cdot(\rho \mathcal{D} \nabla \Lambda)\right]}{-\rho \frac{\chi Z}{2} \partial_{Z}^{2} c} .
$$

The parameter $\Theta$ describes local combustion regimes. It plays the role of a combustion regime index. We stress this is different from the more traditional local flame index, defined through fuel and oxidizer mass fractions as

$$
\xi=\frac{\nabla Y_{O} \cdot \nabla Y_{F}}{\left|\nabla Y_{O}\right|\left|\nabla Y_{F}\right|} .
$$

The present model, based on Eq. (19), serves basically the same purpose as the concept of a flame index, Eq. (20), does. Meanwhile, it selects amongst combustion regimes by considering how chemical source terms are balanced rather than by considering whether gradients of fuel and oxidizer align. The model has been properly validated by simulations of a triple flame [3], and is actively used in an LES of a premixed swirl burner, see below.

\subsection{Partially Premixed Combustion}

Based on the combustion regime index presented above, a consistent model for partially premixed combustion has been developed and validated in simulation of a low swirl burner $[2$, 3]. The specific burner/mixture simulated in [3] are duplicates of that studied in [10]. The LES was performed using our in-house solver [1]. The LES sub-filter stress tensor and sub-filter scalar flux are described using a dynamic Smagorinsky model that employs a Lagrangian-type statistical averaging procedure [11].

The chemical reactions in the flow were treated using a combined flamelet progress variable $[9,12]$ and level set $[2,3,6,13]$ models. This combined model is an example of a presumedPDF (probability density function) approach to turbulent combustion. In the model, the joint sub-filter scale $\operatorname{PDF}(\widetilde{P}(Z, c))$, that describe sub-filter distributions of the mixture fraction and progress variable, is presumed to depend on the filtered mixture fraction $\widetilde{Z}$, the filtered progress variable $\widetilde{c}$, and the mixture fraction variance $\widetilde{Z^{\prime \prime 2}}$. With $\widetilde{P}(Z, c)$ known, any arbitrary filtered reactive variable can be evaluated as

$$
\widetilde{\phi}_{i}=\int_{Z} \int_{c} \phi_{i}(Z, c) \widetilde{P}\left(Z, c ; \widetilde{Z}, \widetilde{Z^{\prime \prime 2}}, \widetilde{c}\right) d Z d c .
$$

Prior to running the LES, the values $\widetilde{\phi}_{i}$ are be pre-computed and stored in a table as functions of $\widetilde{Z}, \widetilde{c}$, and $\widetilde{Z^{\prime \prime 2}}$. Then, they can be accessed as required within the simulation. 
The quantities $\phi_{i}(Z, c)$ are treated as combustion regime-dependent. Therefore, two separate chemistry tables, each of the form $\widetilde{\phi}_{i}=\widetilde{\phi}_{i}\left(\widetilde{Z}, \widetilde{Z^{\prime \prime 2}}, \widetilde{c}\right)$, are created to be used in the LES. The non-premixed table is created by solving the steady flamelet equations for $\phi_{i}(Z, c)$. The premixed table is created by solving the 1D steady premixed flame equations for $\phi_{i}(Z, c)$. Once the values $\phi_{i}(Z, c)$ are known, they are applied in Eq. (35), and the tables are created. At all times and for each mesh points in the LES, one of these two tables is selected based on the combustion regime index $(\Theta)$ defined in the previous section.

Then, in the LES, the scalar transport equations for the filtered mixture fraction and the filtered progress variable are solved for

$$
\begin{gathered}
\frac{\partial \bar{\rho} \widetilde{Z}}{\partial t}+\frac{\partial}{\partial x_{i}}\left(\bar{\rho} \widetilde{u}_{i} \widetilde{Z}\right)=\frac{\partial}{\partial x_{i}}\left(\bar{\rho}\left(\widetilde{D}+D_{t}\right) \frac{\partial \widetilde{Z}}{\partial x_{i}}\right) \\
\frac{\partial \bar{\rho} \widetilde{c}}{\partial t}+\frac{\partial}{\partial x_{i}}\left(\bar{\rho} \widetilde{u_{i}} \widetilde{c}\right)=\frac{\partial}{\partial x_{i}}\left(\bar{\rho}\left(\widetilde{D}+D_{t}\right) \frac{\partial \widetilde{c}}{\partial x_{i}}\right)+\overline{\dot{\omega}_{c}}
\end{gathered}
$$

where the filtered source term for the progress variable $\left(\overline{\dot{\omega}_{c}}\right)$ is evaluated using Eq. 21.

\section{Governing Equations and Numerical Method}

The various laminar and turbulent flames are simulated with the NGA code [1]. The numerical methods characterized by high order conservative finite difference schemes were developed for the simulation of variable density low Mach number turbulent flows. In the framework of LES, the filtered Navier-Stokes and scalar transport equations are

$$
\begin{gathered}
\frac{\partial \bar{\rho}}{\partial t}+\nabla \cdot(\bar{\rho} \widetilde{\mathbf{u}})=0 \\
\frac{\partial \bar{\rho} \widetilde{\mathbf{u}}}{\partial t}+\nabla \cdot(\bar{\rho} \widetilde{\mathbf{u}} \widetilde{\mathbf{u}})=-\nabla \bar{p}+\nabla \cdot \tau \\
\frac{\partial \bar{\rho} \widetilde{\phi}}{\partial t}+\nabla \cdot(\bar{\rho} \widetilde{\mathbf{u}} \widetilde{\phi})=\nabla \cdot \mathbf{g}+\overline{\dot{\omega}}_{\phi}
\end{gathered}
$$

where the turbulent stress tensor and scalar flux terms are expressed using a standard Smagorinsky model

$$
\begin{gathered}
\tau=\bar{\rho}\left(\widetilde{\nu}+\nu_{t}\right)\left(\nabla \mathbf{u}+\nabla \mathbf{u}^{t}\right), \\
\mathbf{g}=\bar{\rho}\left(\widetilde{D}_{\phi}+D_{\phi}^{t}\right) \nabla \widetilde{\phi} .
\end{gathered}
$$

The turbulent eddy viscosity $\left(\nu_{t}\right)$ and diffusivities $\left(D_{\phi}^{t}\right)$ are evaluated using the Lagrangian dynamic sub-grid scale model by Meneveau et al. [14] and extended for variable density and to include the modeling of the turbulent eddy diffusivity by Reveillon and Vervisch [15]. 


\section{Validation: Premixed Swirl Flames and Partially Premixed Combustion}

\subsection{Turbulent Swirling Flames}

In the present project we are studying fuel-flexible combustors including that of advanced next-generation gas turbines. Frequently, the modern gas turbine combustors use swirling lean-premixed flames that offer a possibility of controlled flame temperature and thus reduce $\mathrm{NO}_{x}$ emissions. One more problem are greenhouse gases lading to global climate change. As we stressed above, a way to reduce the emissions of greenhouse gases from coal power plants is to organize an integrated gasification combined cycle. The basic idea of IGCC is to extract syngas from coal. When $\mathrm{CO}$ is shifted to $\mathrm{CO}_{2}$ in the gasifier and separated for sequestration, the gas turbine operates on practically pure $\mathrm{H}_{2}$. For this reason, studies of hydrogen swirling flames is of primary importance.

Turbulent swirling flames (especially hydrogen ones) are inherently unstable. This may lead to noise, flame pulsations and even structural damage. For this reason, flame dynamics in a swirl burner is an important engineering problem, and is a subject of detailed investigations - experimental, theoretical and numerical. In particular, premixed swirl burners have been studied experimentally in [16-19] and numerically in [10]. These burners are particularly notable because they generate lean premixed flames that are quite stable. Thus, when considered in conjunction with the characteristically low levels of $\mathrm{NO}_{x}$ that are produced by lean premixed burning, this inherent stability makes these burners an attractive option in various industrial setups.

Both LES approaches discussed in the previous subsections were validated and applied to simulation of a low swirl burner $[2,3]$. The specific burner/mixture simulated in [3] are duplicates of that studied in [10]. The LES was performed using our in-house solver [1]; see above. The LES sub-filter stress tensor and sub-filter scalar flux are described using a dynamic Smagorinsky model that employs a Lagrangian-type statistical averaging procedure [11]. The chemical reactions in the flow were treated using a combined flamelet progress variable $[9,12]$ and level set $[2,3,6,13]$ models. Such a combined model is an example of a presumed-PDF (probability density function) approach to turbulent burning. In the model, the progress variable and the $\operatorname{PDFs} \widetilde{P}(Z, c)$ that describe sub-filter distributions of the mix-

ture fraction are presumed to depend on the filtered mixture fraction $\widetilde{Z}$, the filtered progress variable $\widetilde{c}$, and the mixture fraction variance $\widetilde{Z^{\prime \prime 2}}$. With $\widetilde{P}(Z, c)$ known, an arbitrary filtered reactive variable can be evaluated as in Eq. (21). Prior to the start of the swirl burner LES, the values $\widetilde{\phi}_{i}$ can be pre-computed and stored in a table as functions of $\widetilde{Z}, \widetilde{c}$, and $\widetilde{Z^{\prime \prime 2}}$. They can then be accessed as required within the simulation.

In premixed regimes, where the gradients of the progress variable become poorly resolved, the filtered chemical source is additionally modified using the solution of a level set equation that describes the location of the flame front $[2,13]$. The quantity $\phi_{i}(Z, c)$ is treated as regime-dependent. Therefore, two separate chemistry tables, each of the form 

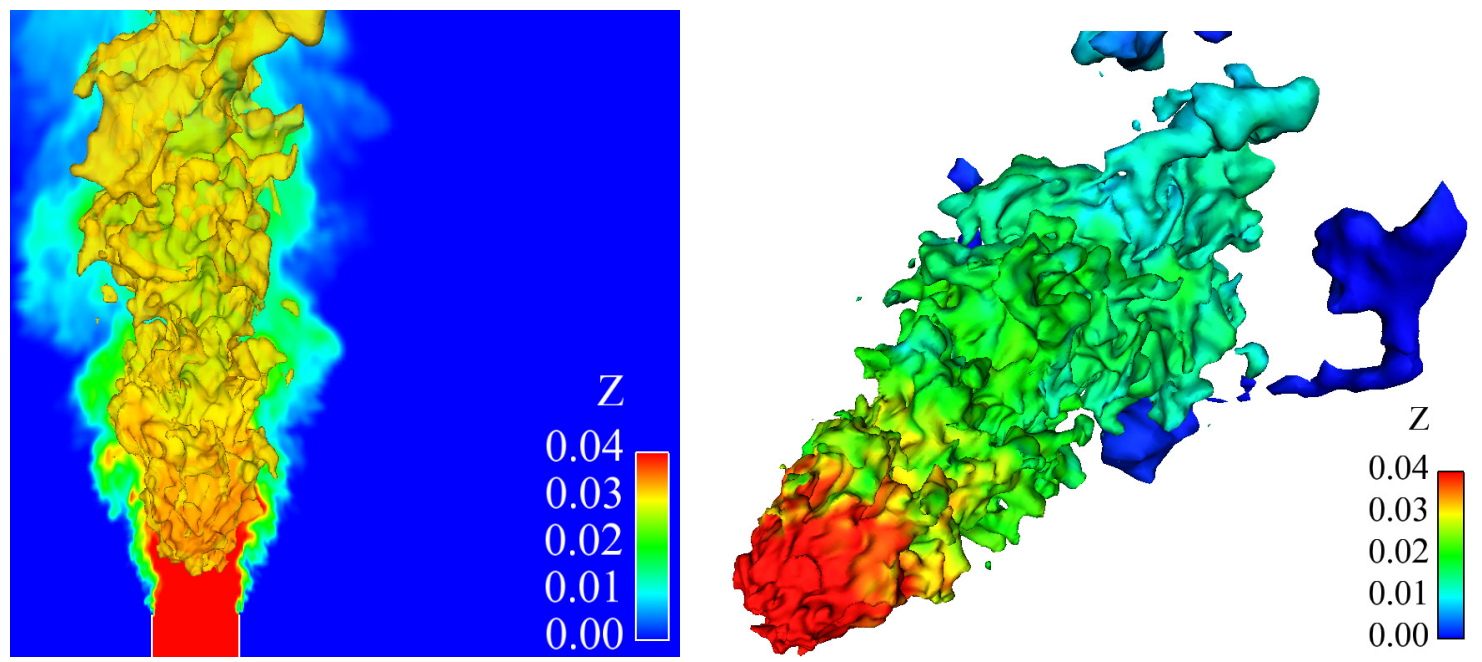

Figure 1: A typical level-set representation of a low-swirl premixed flame [3].

$\widetilde{\phi}_{i}=\widetilde{\phi}_{i}\left(\widetilde{Z}, \widetilde{Z^{\prime \prime 2}}, \widetilde{c}\right)$, are created for usage in the LES. The non-premixed table is created by solving the steady flamelet equations for $\phi_{i}(Z, c)$. The premixed table is created by solving the $1 D$ steady premixed flame equations for $\phi_{i}(Z, c)$. Once the values $\phi_{i}(Z, c)$ are known, they are applied in Eq. (21) and the tables are created. At all times and at all mesh points in the LES, one of these two tables has to be selected using the following two criteria.

The first criterion involves the flammability limits of the local mixture. The steady 1D premixed flame equations only produce realizable burning solutions within a certain range of equivalence ratios. Outside of these limits, valid burning $\phi_{i}(Z, c)$ solutions to the $1 \mathrm{D}$ premixed flame equations do not exist. As a result, no useful information is gained by calling a premixed table outside of these bounds. Thus the non-premixed table is called by default when the local value of $\widetilde{Z}$ is outside of the flammability limits. This criterion is consistent with the combustion regime index (in the sense that it recognizes that the burning velocity appears in the premixed terms). As the flammability limits are reached, the burning velocity approaches zero and the proposed index defaults to predicting nonpremixed combustion. The non-premixed table contains all the non-burning solutions that the premixed table does, but it also contains burning solutions that could account for the fact that heat can diffuse into regions beyond the flammability limits. If the diffused heat were to induce any further combustion beyond these limits, the current approach would predict that the combustion occurs in the non-premixed regime.

The second criterion involves the expression for the proposed combustion regime index. Within the flammability limits, both the premixed and the non-premixed tables provide physically meaningful information. The proposed index can be used to determine which of these tables should be called. Because only filtered quantities are available in LES, the terms that appear in the index must be filtered if the index itself is to be used. Filtering Eq. (18) 
and again neglecting the unsteady terms and the diffusion in $\Lambda$ space gives

$$
\left(\widetilde{\partial_{\Lambda} c}\left[\widetilde{\rho_{u} S_{L, u}}|\nabla \widetilde{\Lambda}|-\nabla \cdot(\bar{\rho} \widetilde{\mathcal{D}} \nabla \widetilde{\Lambda})\right]\right)_{p}+\left(-\bar{\rho} \frac{\widetilde{\chi}}{2} \widetilde{\partial_{Z}^{2} c}\right)_{p n}=\widetilde{\dot{\omega}}_{c}-\widetilde{\dot{\omega}}_{\mathrm{SFS}},
$$

where the last term

$$
\widetilde{\dot{\omega}}_{\mathrm{SFS}}=\left[-\widetilde{\partial_{\Lambda} c}\left[\widetilde{\rho_{u} S_{L, u}}|\nabla \widetilde{\Lambda}|\right]+\partial_{\Lambda} c\left[\widetilde{\rho_{u} S_{L, u}}|\nabla \Lambda|\right]\right]_{p}+\left[\bar{\rho} \frac{\widetilde{\chi_{Z}}}{2} \widetilde{\partial_{Z}^{2} c}-\bar{\rho} \frac{\widetilde{\chi_{Z}}}{2} \partial_{Z}^{2} c\right]_{p n}
$$

describes the sub-filter scale contributions to the chemical source term budget. Most of the terms in the filtered model, Eq. (29), can be computed using the well established presumedPDF formula (35). The exceptions are $\tilde{\chi}_{Z}$ and the sub-filter terms. In the LES, $\tilde{\chi}_{Z}$ is calculated using a sub-filter turbulent diffusivity and the filtered mixture fraction gradient $\nabla \widetilde{Z}, \widetilde{\chi}_{Z}=2 \mathcal{D}_{t} \nabla \widetilde{Z} \cdot \nabla \widetilde{Z} \quad[20]$. The sub-filter terms in Eq. (30) are neglected for now. The present LES implementation can therefore be treated as a check on the budget of an artificially strengthened source term. The strengthening occurs because the term $\widetilde{\dot{\omega}}_{\mathrm{SFS}}$ in Eq. (29) is not appropriately removed from $\widetilde{\dot{\omega}}_{c}$.

The method discussed above has been extensively validated in [3]. In particular, the experiments [19] have been reproduced by usage of LES. A characteristic instantaneous level-set representation of a low swirl premixed flame from LES [3], colored by the mixture fraction $Z$, is shown in Fig. 1. Figure 2 shows time averaged mean (top) and rms (bottom) velocity profiles as a function of the burner radial coordinate. Markers show the experimental results [19]; two experimental data sets shown in each graph represent information from the two sides of the burner centerline (so, the flow field is not completely homogeneous in the azimuthal direction). LES [3] is presented in Fig. 2 by the solid lines. The plane is taken at the distance $0.7 \mathrm{D}$ from the nozzle in all plots. One can see that LES statistics agree very well with experimentally measured ones thus justifying the approach.

\subsection{Lean-Premixed Low-Swirl Hydrogen Flames}

We have applied the present approach to lean-premixed low-swirl hydrogen combustion. The basic idea was to reproduce with LES the results of extensive DNS from LBNL. A low swirl burner with nozzle diameter $D=5 \mathrm{~cm}$, average axial flow velocity $\left|U_{0}\right|=6.34 \mathrm{~m} / \mathrm{s}$ and uniform axial co-flow $0.35 \mathrm{~m} / \mathrm{s}$ is used both in DNS and LES. The mean axial, radial and azimuthal initial velocity profiles are shown in Fig. 3. Such a flow has been perturbed by a certain field of velocity fluctuations. A transition from DNS to LES inflow conditions is given by [21]

$$
\begin{gathered}
U_{L E S}(t)=U_{D N S}(t)+\left[U_{D B}(t)-\left\langle U_{D B}(t)\right\rangle\right] \frac{U_{r m s, D N S}}{U_{r m s, D B}}, \\
V_{L E S}(t)=V_{D N S}(t)+\left[V_{D B}(t)-\left\langle V_{D B}(t)\right\rangle\right] \frac{V_{r m s, D N S}}{V_{r m s, D B}}, \\
W_{L E S}(t)=W_{D N S}(t)+\left[W_{D B}(t)-\left\langle W_{D B}(t)\right\rangle\right] \frac{W_{r m s, D N S}}{W_{r m s, D B}},
\end{gathered}
$$



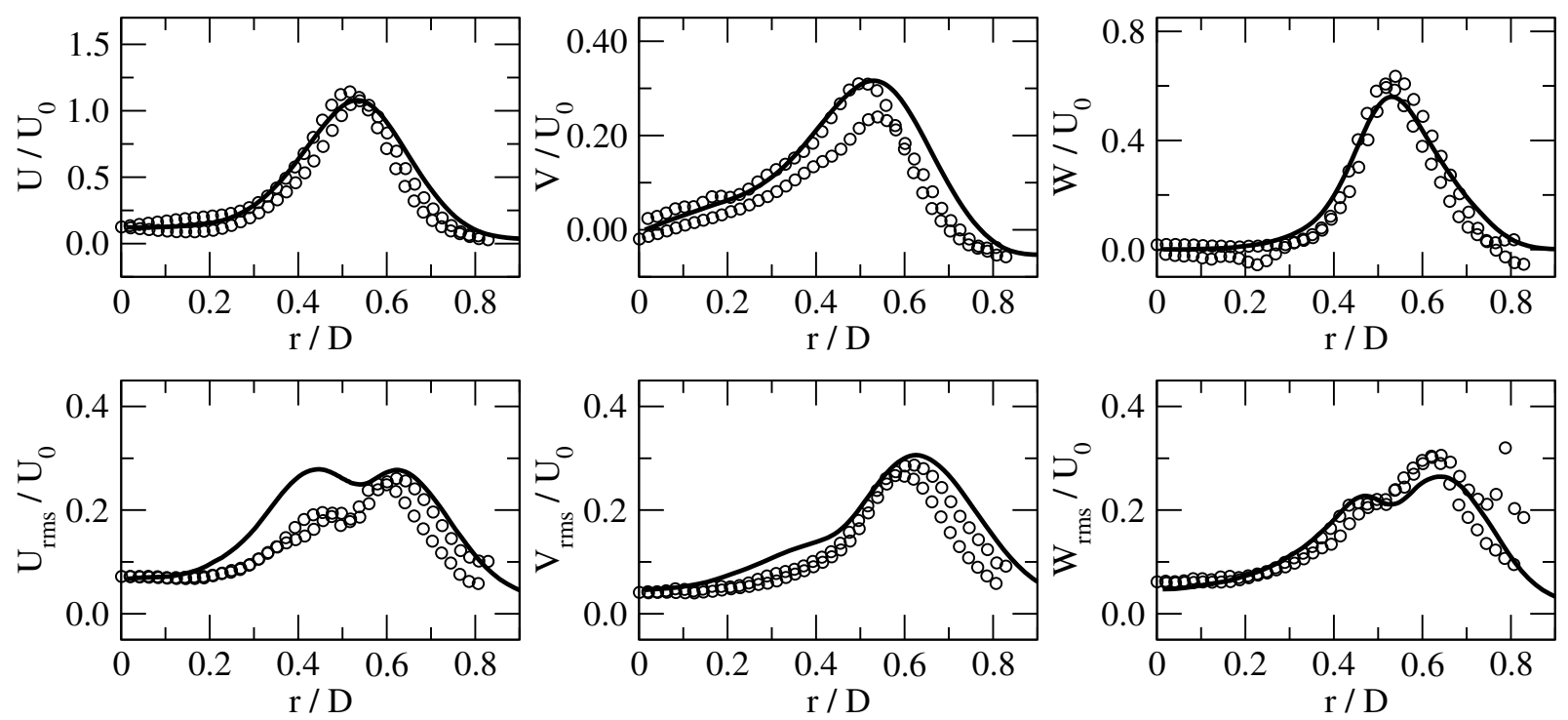

Figure 2: Time averaged swirl burner velocity (top) and rms-velocity (bottom) profiles for methane-air burning. Left-to right: axial, radial, and azimuthal profiles. Solid lines are related to the LES [3]; the experimental results [19] are shown by markers. The planes are taken at the distance $0.7 \mathrm{D}$ form the nozzle.

where $U, V, W$ denote axial, radial and azimuthal velocity components, respectively. The label "DB" in Eqs. (31) - (33) means the velocity profile taken from a certain turbulent database; we are using that of [3].

\subsubsection{LES of a Cold Swirling Flow}

First, we perform LES of a cold (non-reactive) flow in such a configuration. An instantaneous total velocity field in that case is shown in Fig. 4. Snapshots (a) - (c) present radial planes taken at the nozzle, and at $5 \mathrm{~cm}$ and $15 \mathrm{~cm}$ from the nozzle; r-z plane is shown in snapshot (d). The quantity of the total flow velocity is shown by the color: from $0.35 \mathrm{~m} / \mathrm{s}$ (blue) to $12 \mathrm{~m} / \mathrm{s}$ (red). Figure 5 presents a characteristic 3D isosurface of the flow velocity field, $|\mathbf{U}|=U_{0}=6.34 \mathrm{~m} / \mathrm{s}$. Both figures 4,5 demonstrate that moving far from the nozzle we get weaker but wider flow. Figure 6 shows time averaged mean (left) and rms (right) velocity profiles as a function of the burner radial coordinate. The averaging radial plane is taken at the distance $0.5 \mathrm{D}$ from the nozzle. Axial, radial and azimuthal profiles are shown top-tobottom. Similar to Fig. 2, all values in Fig. 6 are scaled by the average axial flow velocity, which is $U_{0}=6.34 \mathrm{~m} / \mathrm{s}$ in the present case. The mean axial velocity definitely dominates over the mean radial and azimuthal profiles. At the same time, the roles of $U_{r m s}, V_{r m s}$, and $W_{r m s}$ are comparable. Sharp peaks and cusps in the plots for rms-velocities may claim that the simulation time was relatively short to determine the mean rms profiles (i.e. we did not get/keep statistically-stationary turbulence). At present, we are going to take averaging over longer time in order to determine the profiles in Fig. 6 better. Besides, we would like 


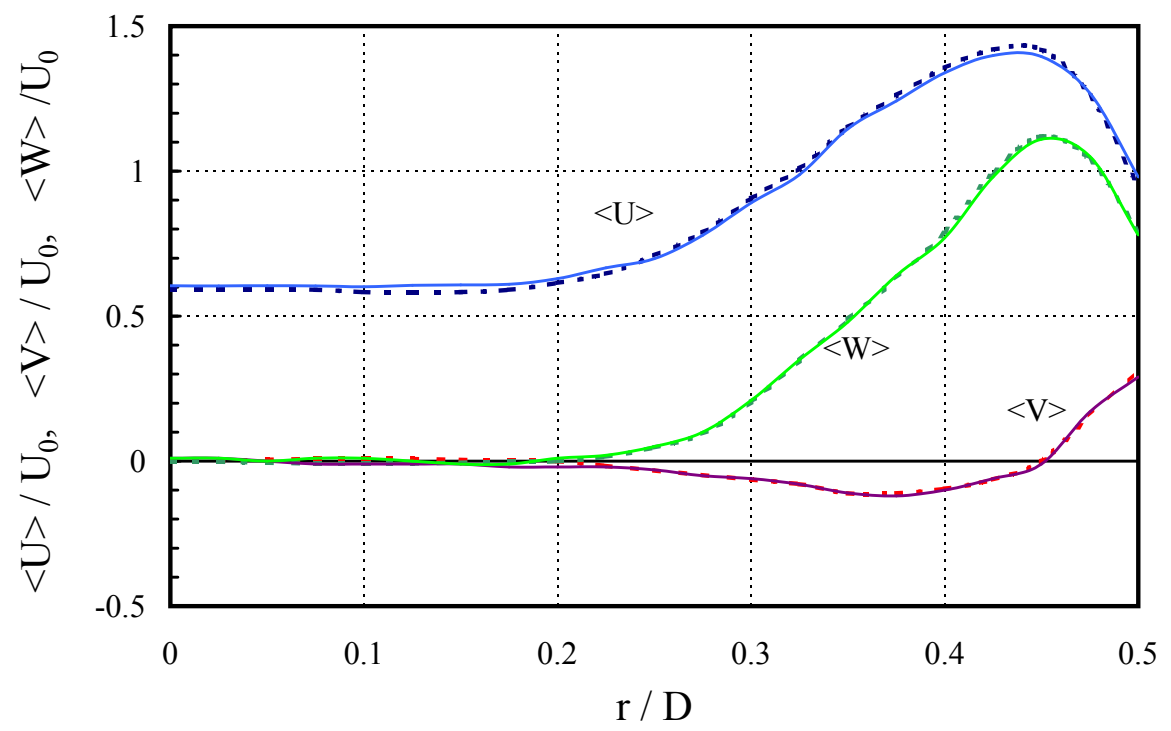

Figure 3: Mean inflow velocity profiles (axial, radial and azimuthal): DNS (dashed) and LES (solid).

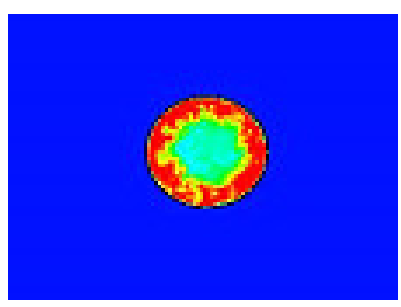

(a) nozzle

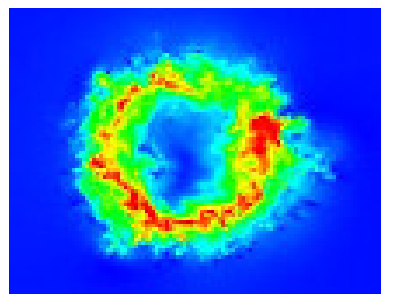

(b) $5 \mathrm{~cm}$

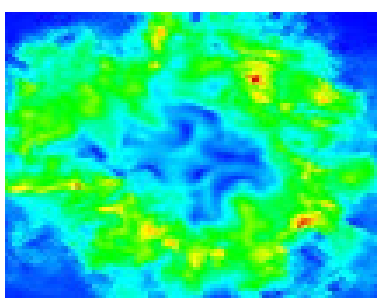

(c) $15 \mathrm{~cm}$

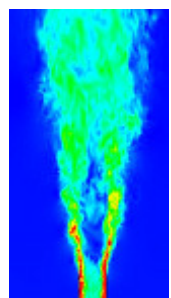

(d) r-z plane

Figure 4: Total velocity field in hydrogen burning: from $0.35 \mathrm{~m} / \mathrm{s}$ (blue) to $12 \mathrm{~m} / \mathrm{s}$ (red). Snapshots (a) - (c) show three radial planes taken at 0,5, $15 \mathrm{~cm}$ from the nozzle; snapshot (d) presents r-z plane. 


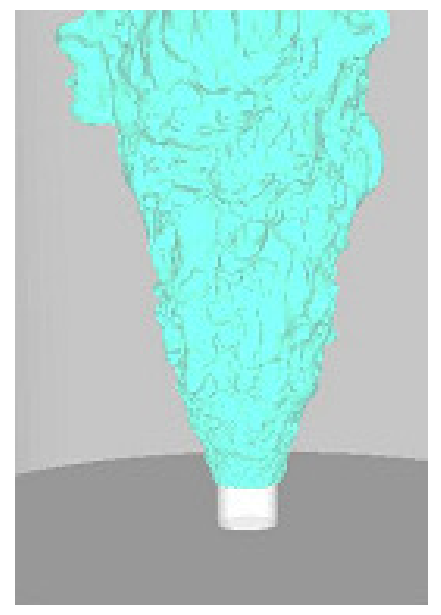

Figure 5: Characteristic flow velocity isosurface; $|\mathbf{U}|=6.34 \mathrm{~m} / \mathrm{s}$.

to compare our "cold" statistics with that of DNS performed at LBNL. This work is now in progress.

\subsubsection{Reacting Flow}

Similar to LBNL's DNS, we also perform LES of lean premixed hydrogen burning with $\phi=0.37$. Figure 7 shows two characteristic 3D flame isotherms taken at $T=900 \mathrm{~K}$ (a) and $T=1200 \mathrm{~K}(\mathrm{~b})$. In both cases we observe strongly corrugated (turbulent) flame shape resulted from swirling. A typical flame evolution in $\mathrm{r}-\mathrm{z}$ plane is shown in Fig. 8. The temperature distribution is presented by the color: from $300 \mathrm{~K}$ (white) to $1310 \mathrm{~K}$ (red). Again, we observe strongly corrugated flames in all four snapshots. Figure 9 demonstrates the same result. Actually, the snapshot of $\mathrm{H}_{2} \mathrm{O}$ mass fraction shown in Fig. 9 resembles snapshots of Fig. 8 quite well. Figure 10 is a counterpart of Fig. 4 for a reacting flow. Total velocity field in a lean-premixed low-swirl hydrogen burning with $\phi=0.37$ is shown by the color: from $0.35 \mathrm{~m} / \mathrm{s}$ (white) to $12 \mathrm{~m} / \mathrm{s}$ (red). Again, snapshots (a) - (c) show three radial planes taken at $0,5,15 \mathrm{~cm}$ from the nozzle, while snapshot (d) presents r-z plane. We would like to stress that plots of Fig. 10 resemble that of Fig. 4 quite well. Comparing Figs. 6 and 11, where reacting flow statistics is shown, we may come to the same conclusion. Indeed, Fig. 11 presents time-averaged velocity and temperature profiles. The mean values are shown in the left column; rms parameters are presented in the right column. Axial, radial and azimuthal velocities, and temperature are shown top-to bottom. Again the averaging is performed in the radial plane taken at the distance $0.5 \mathrm{D}$ form the nozzle. Of course, Figs. 10, 11 differs quantitatively from Figs. 4, 6, respectively. However, qualitative difference between the "cold" and "reacting" cases is hardly seen. Furthermore, the mean values of velocities and rms-velocities are quite close in both cases. It means that the effect of leanpremixed hydrogen burning on the velocity and vorticity is much less than the role of swirling. This result is different from that of methane-air burning [3,19], and may may be explained 

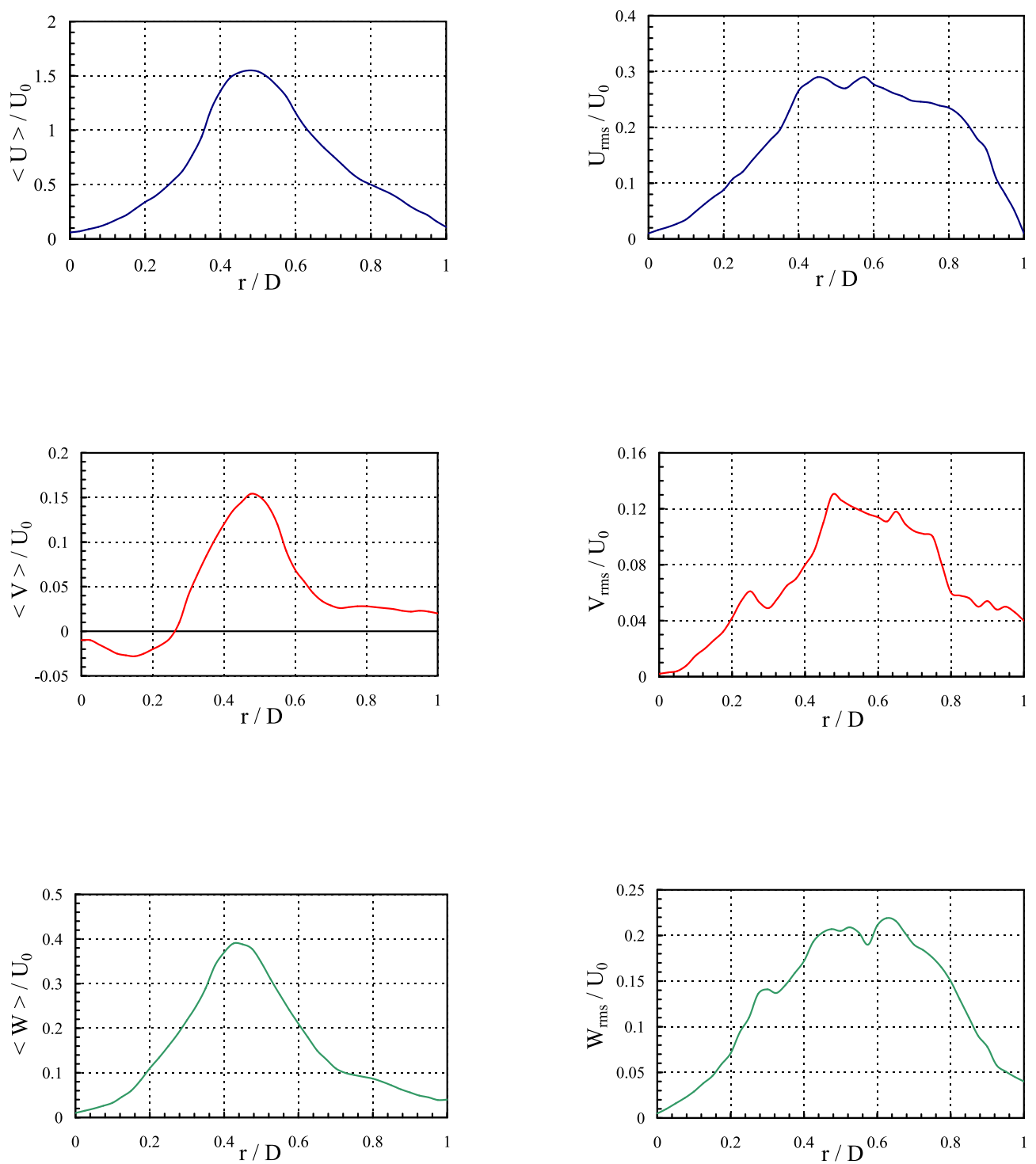

Figure 6: Time averaged swirl burner velocity (left) and rms-velocity (right) profiles in a non-reactive flow. Axial, radial and azimuthal velocities are shown top-to-bottom; $D=5 \mathrm{~cm}$ is the nozzle diameter, $U_{0}=6.34 \mathrm{~m} / \mathrm{s}$ is the average axial flow velocity. 


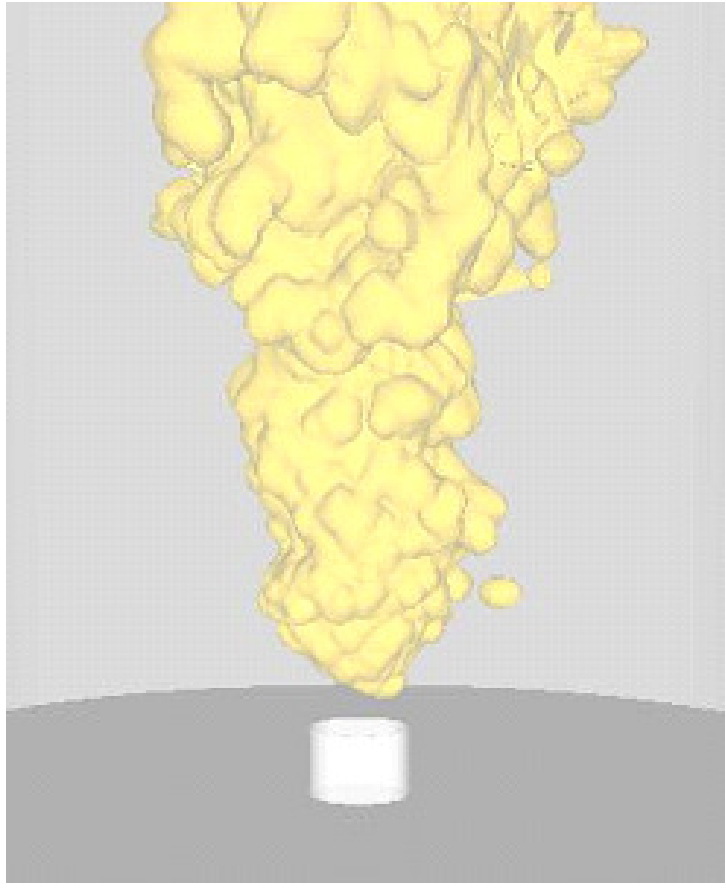

(a) $\mathrm{T}=900 \mathrm{~K}$

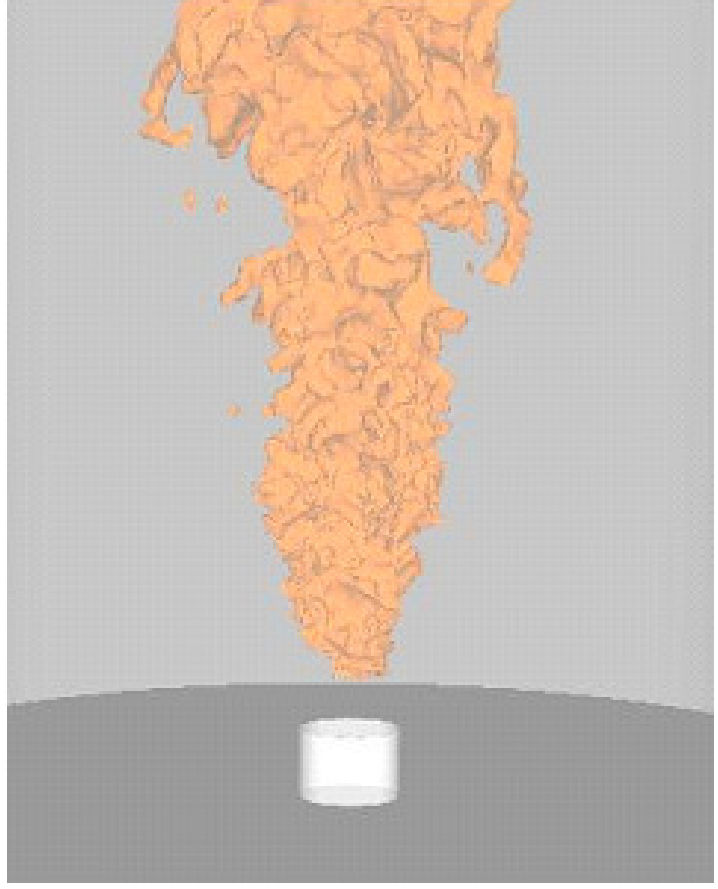

(b) $\mathrm{T}=1200 \mathrm{~K}$

Figure 7: Characteristic flame shape shown by isotherms $\mathrm{T}=900 \mathrm{~K}$ (a) and $\mathrm{T}=1200 \mathrm{~K}$ (b).
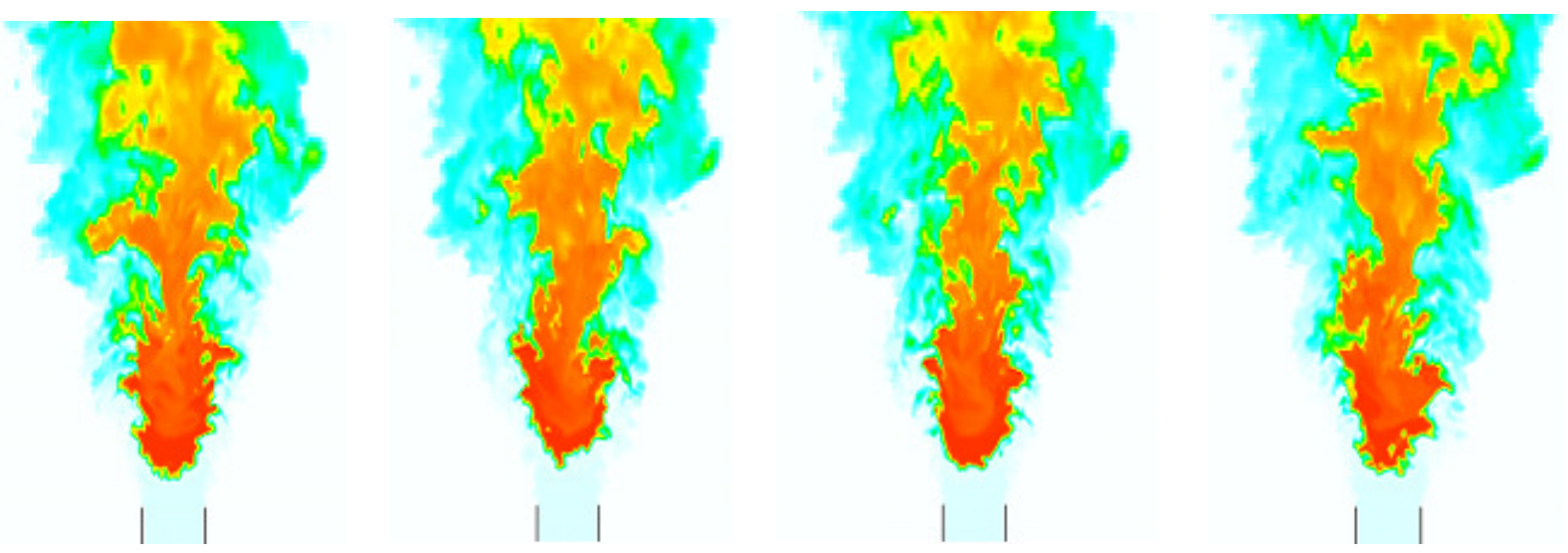

Figure 8: Evolution of a swirling premixed hydrogen flame with $\phi=0.37$. The temperature field is shown by the color: from $300 \mathrm{~K}$ (white) to $1310 \mathrm{~K}$ (red). 


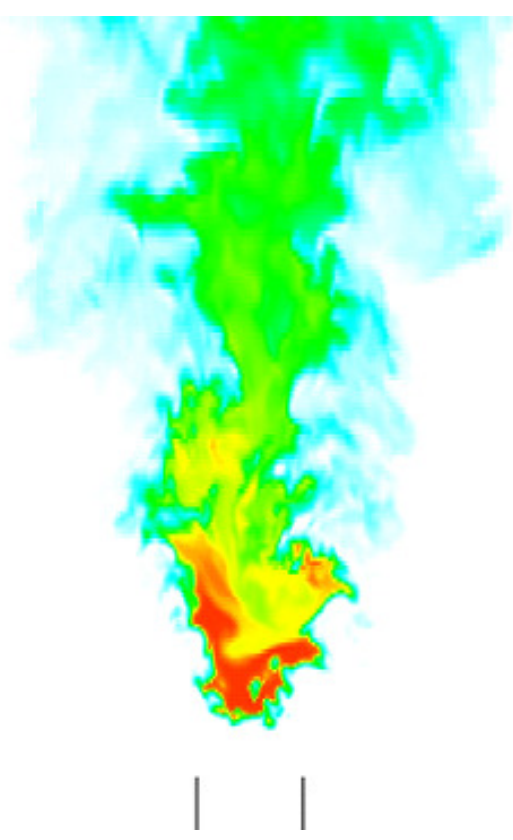

Figure 9: Characteristic snapshot of $\mathrm{H}_{2} \mathrm{O}$ mass fraction.

as follows. The upstretched laminar flame speed for lean hydrogen burning with $\phi=0.37$ is low, $S_{L}=0.104 \mathrm{~m} / \mathrm{s}$. The thermal expansion in burning is also rather small in that case, $\Theta=T_{b} / T_{f} \approx 4.4$. As a result, the characteristic local flow generated by the flame propagation is $S_{L}(\Theta-1) \approx 0.353 \mathrm{~m} / \mathrm{s}$, which is much less than the characteristic rms velocity $0.5-2 \mathrm{~m} / \mathrm{s}$, and the mean velocity $2-13 \mathrm{~m} / \mathrm{s}$. Thus swirling dominates over the flame-generated flow.

\section{Simulation of a Low Swirl Combustor}

A low-swirl combustor of NETL and LBNL is a main test case in the present project. In this configuration, experimental measurements of several flames with various fuel composition are performed so that the effects of hydrogen content in methane/hydrogen/air combustion can be investigated systematically.

\subsection{Cold Flow Simulation}

In the present section, we present the numerical results of the simulation of the cold flow in a low-swirl injector. Full details about the experimental configuration can be found in Ref. [22].

Figure 12 shows the instantaneous velocity and vorticity profiles in this configuration. In this experimental setup, the air flow is injected through a central unswirled channel and a swirl annulus. Then, the air enters the chamber where it forms a recirculation region. This recirculation region is one of the mechanisms which ensures the stability of the burner 


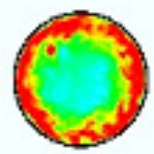

(a) nozzle

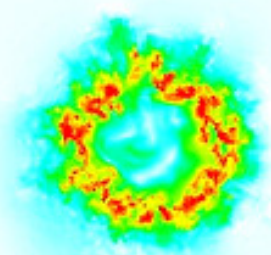

(b) $5 \mathrm{~cm}$

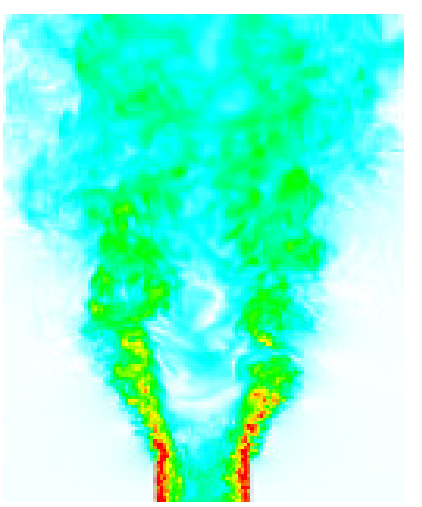

(d) r-z plane

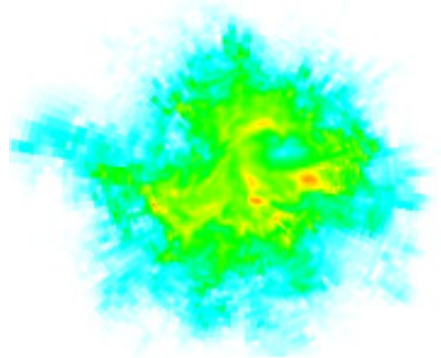

(c) $15 \mathrm{~cm}$

Figure 10: Total velocity field in hydrogen burning: from $0.35 \mathrm{~m} / \mathrm{s}$ (white) to $12 \mathrm{~m} / \mathrm{s}$ (red). Snapshots (a) - (c) show three radial planes taken at 0, 5, $15 \mathrm{~cm}$ from the nozzle; (d) presents r-z plane. 

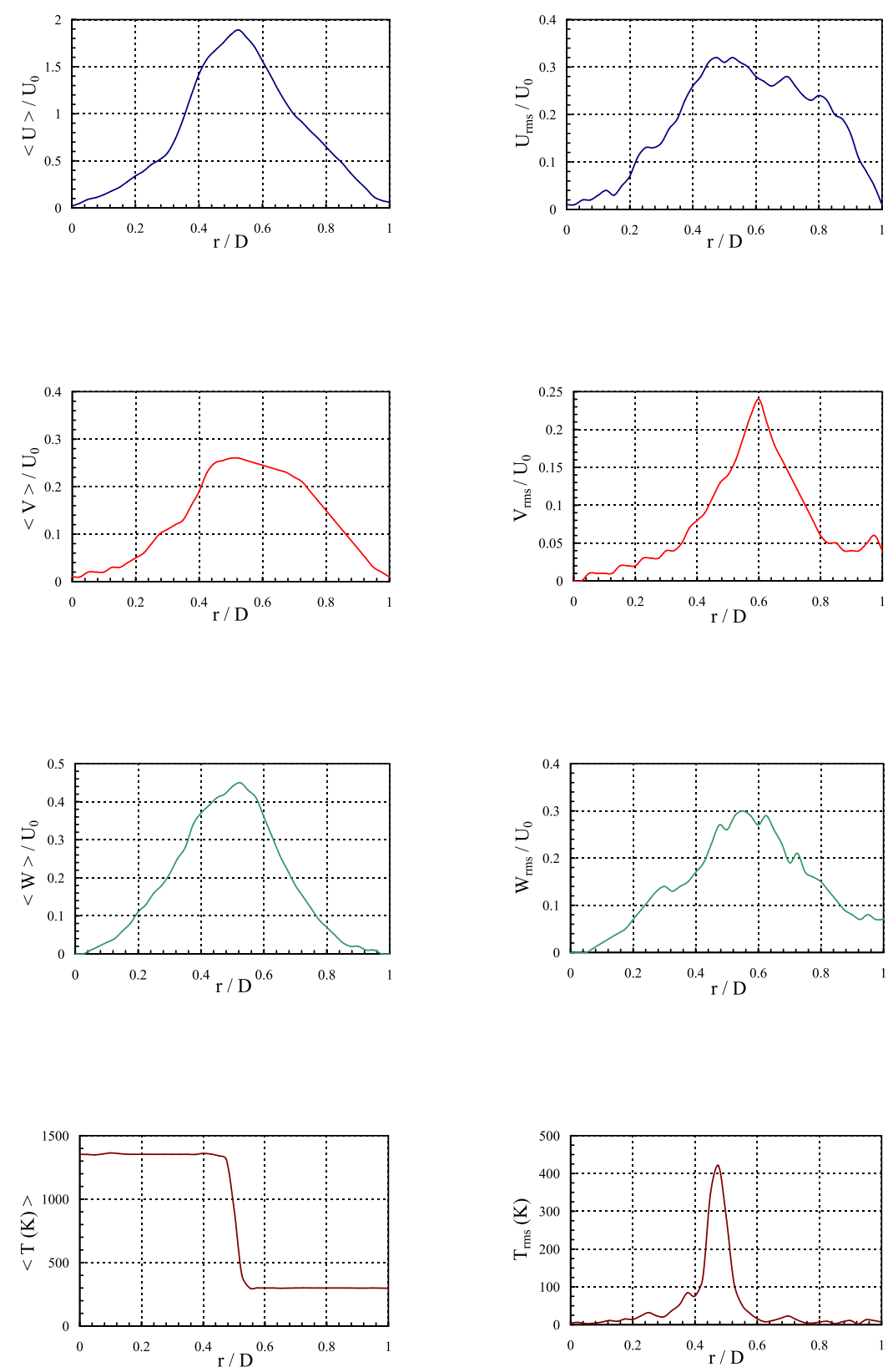

Figure 11: Time averaged swirl burner velocity and temperature profiles for lean-premixes hydrogen burning with $\phi=0.37$ (left). The right column shows rms-parameters. Axial, radial and azimuthal velocities, and temperature are shown top-to-bottom in both columns. 

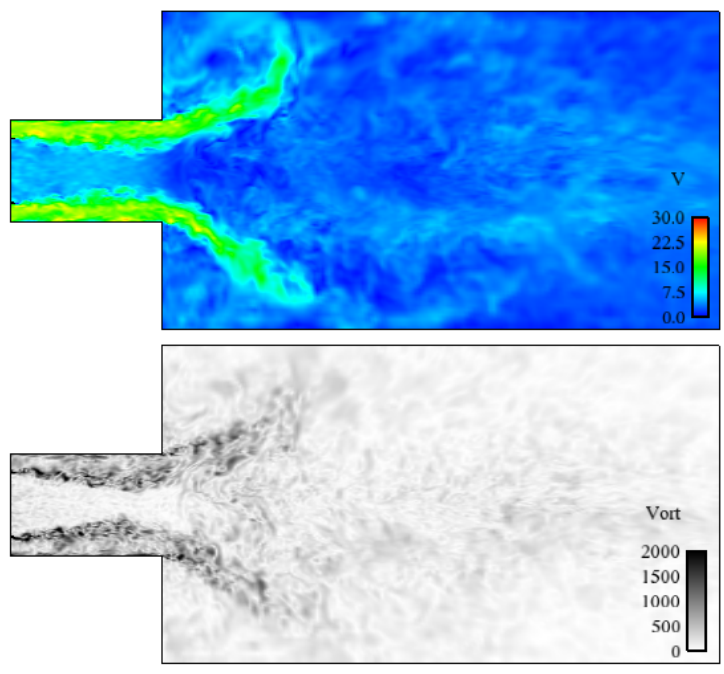

(a) Instantaneous velocity \& vorticity

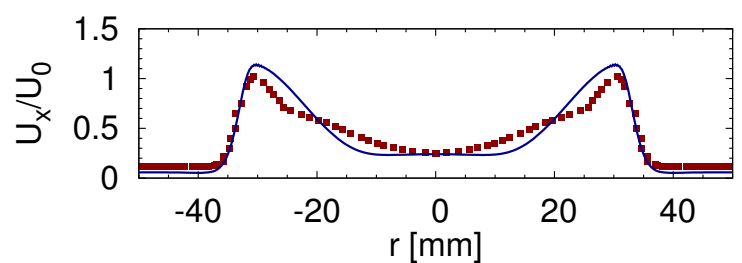

(b) Mean axial velocity $(x=6.5 \mathrm{~mm})$

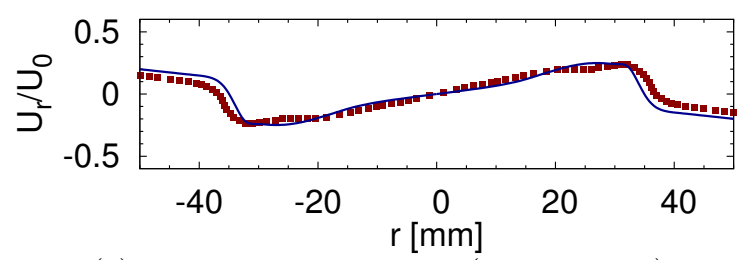

(c) Mean radial velocity $(x=6.5 \mathrm{~mm})$

Figure 12: Results of the cold flow simulation of the low swirl burner and comparison with experimental data [22].

configuration. Figure 12 also shows a comparison of the mean axial and radial velocity with experimental measurements at a station inside the combustion chamber. As it can be observed, the present numerical simulation performs reasonably well in capturing the recirculation region.

During the numerical simulation of the cold flow, the results were found to be very sensitive to the inlet boundary conditions. Similar senstivities were also found in our previous investigation of a lean methane premixed flame [3]. As a result, a different burner with well defined boundary conditions was recently chosen for the numerical simulations of the reacting flows.

\subsection{Methane Flame}

In order to validate the numerical procedure and modeling approach, the case of a lean premixed methane/air flame $(\phi=0.59)$ is considered first. Methane is taken here as an example of unity Lewis number fuel. The goal is to validate the treatment of the boundary conditions and to ensure that the simulation of turbulent premixed flame with unity Lewis number is fuel is described accurately before investigating the combustion of non-unity Lewis number fuels in turbulent premixed flames.

As mentioned previously, the reacting flow simulations were performed using a different burner geometry than the cold flow. Cheng et al. [23] reported the combustion characteristics of various lean premixed low-swirl injectors with different fuels including pure methane and pure hydrogen. As the combustion chamber is closed, this experimental setup corresponds to fully premixed combustion. In this section, the case of the purely premixed methane flame $(\phi=0.59)$ has been investigated, and the results are presented.

In order to properly characterize the flow entering the combustion chamber, it was found 

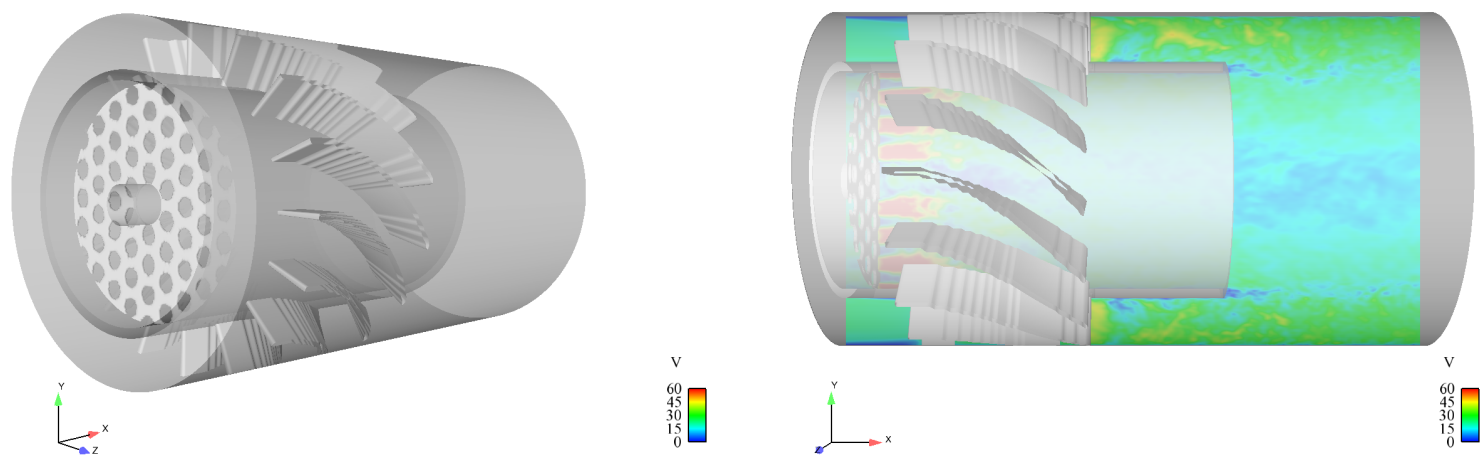

Figure 13: Full details of the injector geometry (left) and instantaneous profiles of velocity (right) for the simulation of the lean methane premixed low-swirl injector.

necessary to resolve the flow through the injector. As a result, the numerical investigation of the lean premixed methane burner was decomposed into two distinct simulations.

The first simulation corresponds to the investigation of the flow through the injector. In order to properly describe the complex details of the injector, the geometry was represented with Immersed Boundaries (IB). Figure 13 shows the injector composed of a swirled annulus composed of sixteen vanes and an unswirled central pipe through a perforated plate. Figure 13 also shows an instantaneous velocity profile. As a result of the complex details of the geometry, the flow exhibits strong non-uniformity and unsteadiness. This simulation was performed with 8 millions grid points on a cylindrical mesh.

The second simulation corresponds to the flow inside the combustion chamber. The inlet boundary conditions for this simulation were extracted from the numerical simulation of the full injector presented previously. Figure 14 shows the instantaneous profiles of density, temperature, and velocity in the combustion chamber. As expected, a strong recirculation region forms early on in the chamber and stabilizes the flame front. The flame exhibits a common "M" shape characteristic of attached premixed flames.

The first step to performing the reacting flow simulations consists in building a premixed chemical table. A single 1D freely propagating laminar flame with the same equivalence ratio was simulated with detailed chemistry [24], and the various chemical properties mapped as a function of the progress variable. For methane, the progress variable was defined as the sum of $\mathrm{CO}_{2}, \mathrm{CO}, \mathrm{H}_{2} \mathrm{O}$, and $\mathrm{H}_{2}$ mass fractions. The computed laminar burning velocity is $S_{L}=10.8 \mathrm{~cm} / \mathrm{s}$. The simulation of the combustion chamber was performed on a cylindrical mesh of $N_{x} \times N_{r} \times N_{\theta}=192 \times 160 \times 64$ grid points with 48 cpus.

Figure 15 shows the temperature and velocity fields of the methane flame. Under high velocity and high swirl, the flow at the edge of the inner pipe opens up (at an angle close to $45^{\circ}$ ) and creates a recirculation region slightly downstream of the inlet. This recirculation region is the key element responsible for the stabilization of the flame front. The flame exhibit an "M" shape and is anchored at the edges of the inlet pipe. In an enclosed domain, hot gases are entrained in the recirculation regions in the corners and make their way to the 

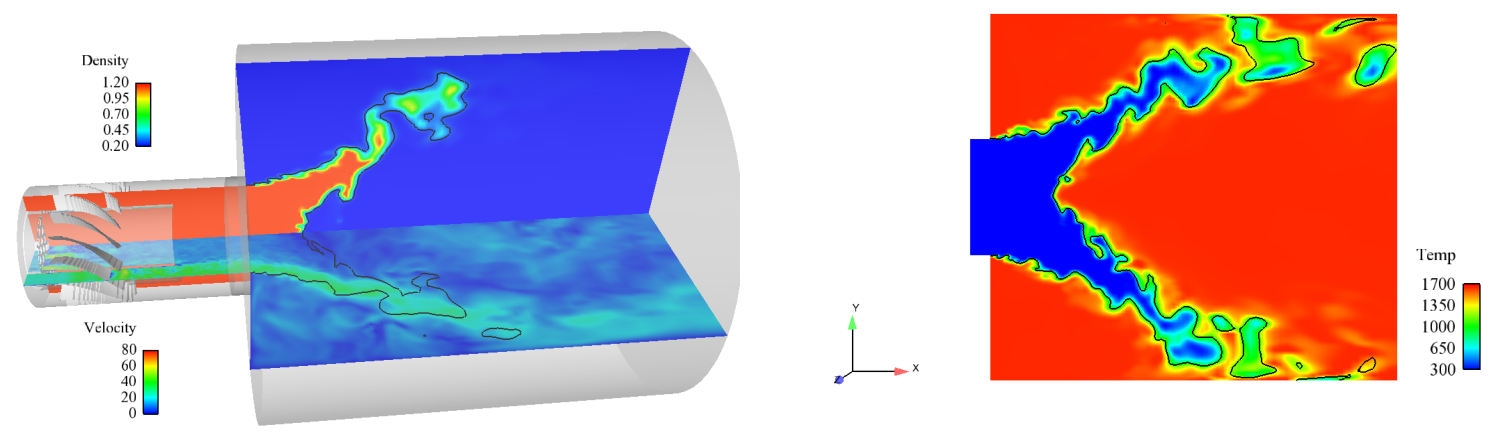

Figure 14: Details of the full geometry including the injector and the combustion chamber (left) and instantaneous profiles of temperature (right) for the simulation of the lean methane premixed low-swirl injector.

shear layers of the jets.

In order to assess the ability of the approach to predict turbulent premixed flames with unity number Lewis fuel, we evaluate time averaged statistics and compare the results with experimental measurements. Figure 16 shows the mean and rms velocity along the centerline. The decay of the mean axial velocity as the jet opens is well reproduced by the numerical simulation. More importantly, the location and magnitude of the recirculation is captured accurately. Similarly, the $2 \mathrm{D}$ velocity fluctuations $\left(q=\sqrt{u_{x}^{\prime 2}+u_{r}^{\prime 2}}\right)$ along the axis exhibits the same flat shape as observed experimentally. While no temperature or species measurements are available to validate the combustion processes themselves, the quality of the velocity predictions is an evidence that the turbulent field and the effects of combustion are modeled properly.

Similar conclusions can be drawn from radial profiles of velocity. Figure 17 shows the mean and rms velocity at three different locations: just before the recirculation region $(30 \mathrm{~mm})$, inside the recirculation region $(50 \mathrm{~mm})$, and further downstream $(80 \mathrm{~mm})$. For the three stations, the comparison with the measurements is favorable. The spreading rate of the swirling jet is captured reasonably well as seen on the profiles of axial and radial velocities. More importantly, second order moments such as the turbulent velocity fluctuations are predicted well by the current approach. Though, some discrepancies in the mean quantities and fluctuations exist. However, the experimental measurements suffer from some uncertainties. For instance, they are not perfectly symmetric and optical access to part of the domain was difficult (for $r<-30 \mathrm{~mm}$ at $x=50 \mathrm{~mm}$ and $x=80 \mathrm{~mm}$ ).

As a result, the present comparison between numerical simulation and experimental measurements is a strong evidence of the ability of the model to model the combustion of close to unity Lewis number fuel such as methane. 


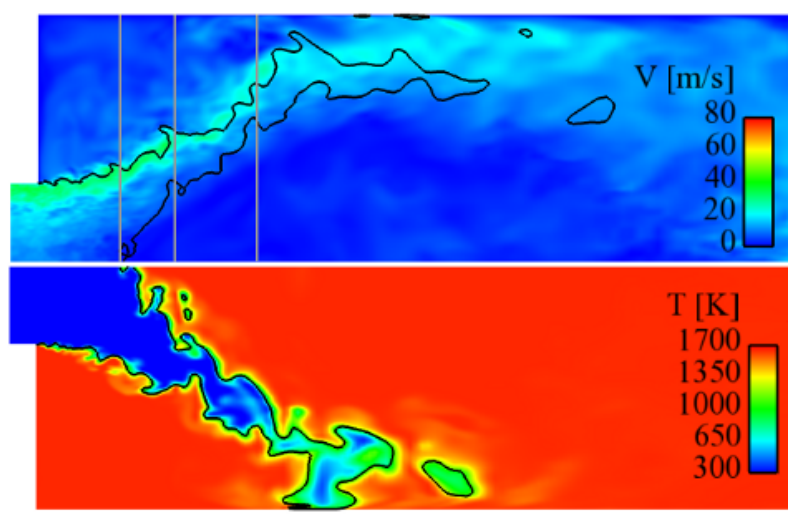

Figure 15: Instantaneous fields of velocity magnitude (top) and temperature (bottom) for the simulation of the turbulent flame of methane/air. An isocontour $\hat{G}=0$ shows the location of the flame front (black line). Grey lines indicate locations where statistics are gathered (30 mm, $50 \mathrm{~mm}$, and $80 \mathrm{~mm})$.

\subsection{Hydrogen Flame}

With confidence in the treatment of the boundary conditions and favorable predictions for methane combustion, it is now possible to investigate the effects of differential diffusion in a turbulent hydrogen flame. The experimental setup is the same as previously used for methane. The only differences reside in the fuel used $\left(\mathrm{H}_{2}\right)$ and the global equivalence ratio $(\phi=0.40)$.

Similarly to methane combustion, a premixed chemical table is created first. This table is build upon a series of 1D laminar premixed flat flames with varying equivalence ratios. The flames considered are shown in Fig. 25. Once again, these flames were computed with detailed chemistry [25] and assuming that the Lewis number of all species is unity with the exception of hydrogen $\left(L e_{H_{2}}=0.3\right)$. The theoretical laminar burning velocity for $\phi=0.4$ is $S_{L}=20.1 \mathrm{~cm} / \mathrm{s}$, and the lean flammability limit is predicted around $\phi=0.27$.

Figure 18 shows some results of the numerical simulation of the lean hydrogen injector. This simulation was performed without the model for non-unity Lewis number combustion in order to assess the ability of the original model to reproduce the large scale features of the flow field. This enclosed low-swirl injector exhibits a typical "M" flame in perfect agreement with experimental observation. The flame is attached to burner surface at the edge and is stabilized by the recirculation region.

Figure 19 and 20 show a comparison of the experimental measurements with the numerical simulations with unity Lewis number assumption. The model with the unity Lewis number does not capture the major characteristics observed in the experiment. 

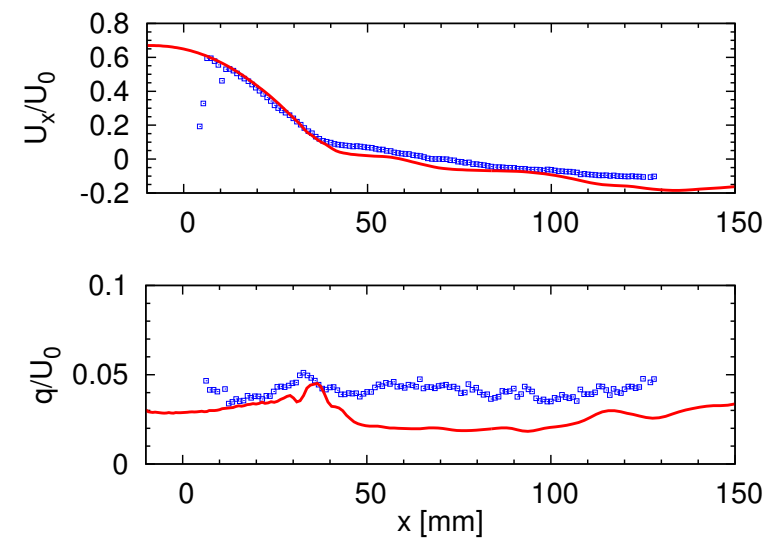

Figure 16: Comparison of mean axial velocity (top) and 2D velocity fluctuations $(q=$ $\sqrt{u_{x}^{\prime 2}+u_{r}^{\prime 2}}$ - bottom) for the methane/air flame with experiments (symbols) along the centerline.

\subsection{Flash-Back and Blow-Out}

As a first step toward the prediction of flash-back and blow-out in hydrogen-enriched flames, we consider the experiments in Cheng et al. [26] and present LES results with the unity Le assumption. In Cheng et al. [26], the equivalence ratio at the flash-back is presented for pure hydrogen and hydrogen/methane flames at elevated pressures and temperatures. Here, a pure hydrogen flame at $0.202 \mathrm{MPa}$ and $530 \mathrm{~K}$ is considered. When the inlet bulk velocity is $20 \mathrm{~m} / \mathrm{s}$, the measured equivalence ratio at flash-back is 0.5 .

Figure 21 shows the instantaneous fields of the progress variable and the velocity fields. The global equivalence ratio is 0.5. As discussed above, the unity Lewis number assumption is used in LES. As in Fig. 21, the flash-back is not correctly predicted. In Fig. 22, LES results for the equivalence ratio of 0.6 are shown. For the equivalence ratio of 0.5 , the laminar burning velocity is $156 \mathrm{~cm} / \mathrm{s}$, while it is $265 \mathrm{~cm} / \mathrm{s}$ for the equivalence ratio of 0.6 . Although the flame length becomes shorter, the flash-back is not observed even for the equivalence ratio of 0.6. This suggests that for the prediction of flash-back in hydrogen and hydrogen-

enriched flames, non-unity Lewis number effects should be considered, for example, using the hydrogen/progress variable or the mixture fraction/progress variable approach.

\section{Model Extension for Hydrogen Combustion}

As shown in the previous section, the state-of-the-art combustion model presented in Sec. 2 does not perform well for lean premixed hydrogen flames. In this section, the extension of the model for lean hydrogen combustion is presented.

The model for premixed combustion relies on a combined flamelet progress variable $[9,12]$ and level set $[2,3,6,13]$ apporach. In this model, the filtered flame front is tracked using the 

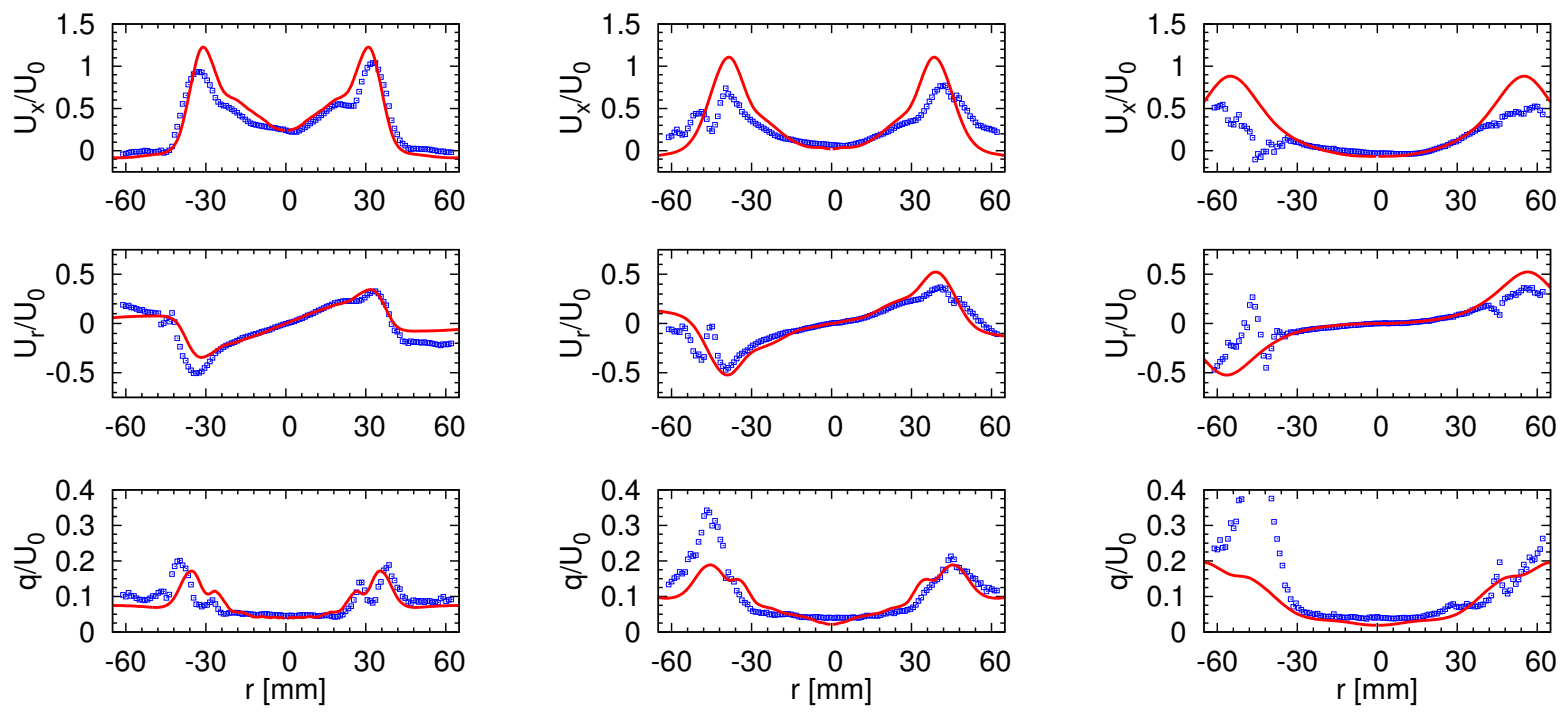

(a) $x=30 \mathrm{~mm}$

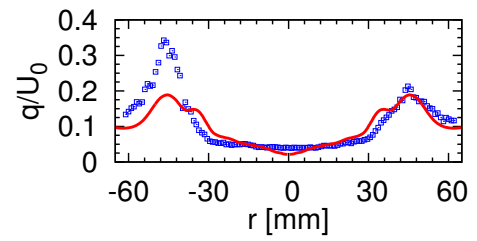

(b) $x=50 \mathrm{~mm}$

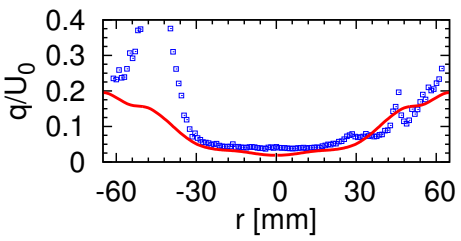

(c) $x=80 \mathrm{~mm}$

Figure 17: Comparison of mean axial velocity (top), mean radial velocity (center), and 2D velocity fluctuations $\left(q=\sqrt{u_{x}^{\prime 2}+u_{r}^{\prime 2}}\right.$ - bottom) for the methane/air flame with experiments (symbols) at three locations downstream of the injection.

level set equation:

$$
\frac{\partial \hat{G}}{\partial t}+\widetilde{\mathbf{u}} \cdot \nabla \hat{G}=\frac{\rho_{u}}{\bar{\rho}} s_{\Delta} \hat{\mathbf{n}} \cdot \nabla \hat{G},
$$

where the level set function $\hat{G}$ is used to describe the location of the filtered flame front, $\widetilde{\mathbf{u}}$ is the (density-weighted) filtered velocity, $\bar{\rho}$ is the filtered density, $\rho_{u}$ is the density of the unburned mixture, $s_{\Delta}$ is the sub-filter burning velocity representing the propagation speed of the iso-surface with $\hat{G}=G_{0}$, and $\hat{\mathbf{n}}$ is the normal vector of the $G_{0}$ surface. The local flame structure is approximated by a laminar flamelet. The density, chemical reaction rates and other thermo-chemical quantities are represented by a small number of parameters that characterize the laminar flamelets. The filtered density and chemical reaction rates that are required for LES are then obtained by

$$
\widetilde{\phi}_{i}=\int_{\mathbf{S}} \phi_{i}(\mathbf{S}) \widetilde{P}(\mathbf{S}) d \mathbf{S}
$$

where $\mathbf{S}$ is the parameters used in the laminar flamelet library. When the Lewis number of the deficient reactant is close to unity, premixed combustion is characterized only by the reaction progress variable, $C$, which is typically defined as normalized temperature or mass fractions of major products. Otherwise, more parameters are needed to capture the non-unity Lewis number effects and variations in the equivalence ratio. The joint sub-filter scale PDF, $\widetilde{P}(\mathbf{S})$, which describes sub-filter distributions of the progress variable and other 

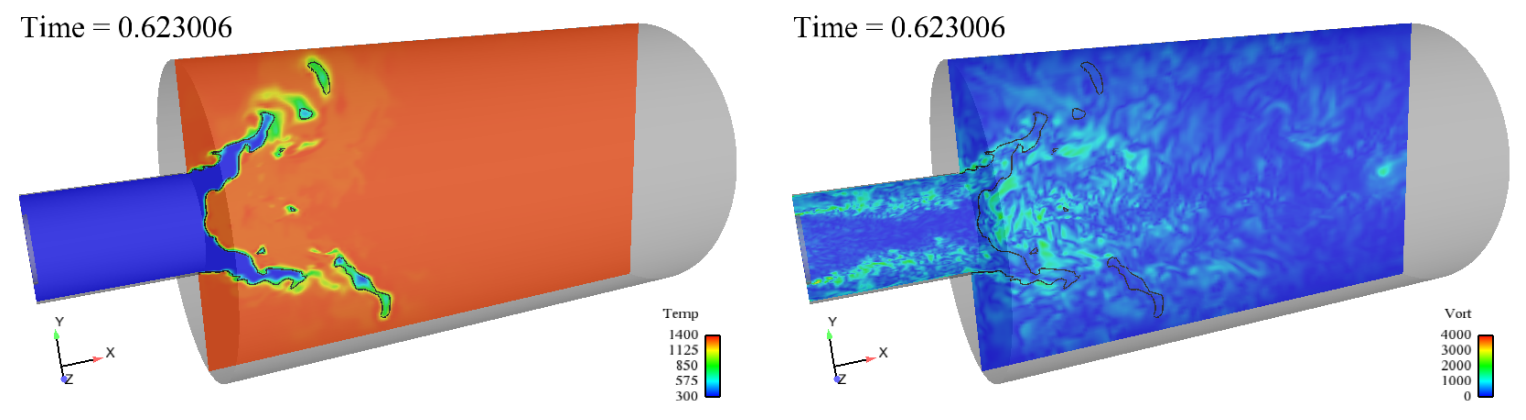

Figure 18: Instantaneous profiles of temperature (left) and vorticity (right) for the simulation of the lean hydrogen premixed low-swirl injector.
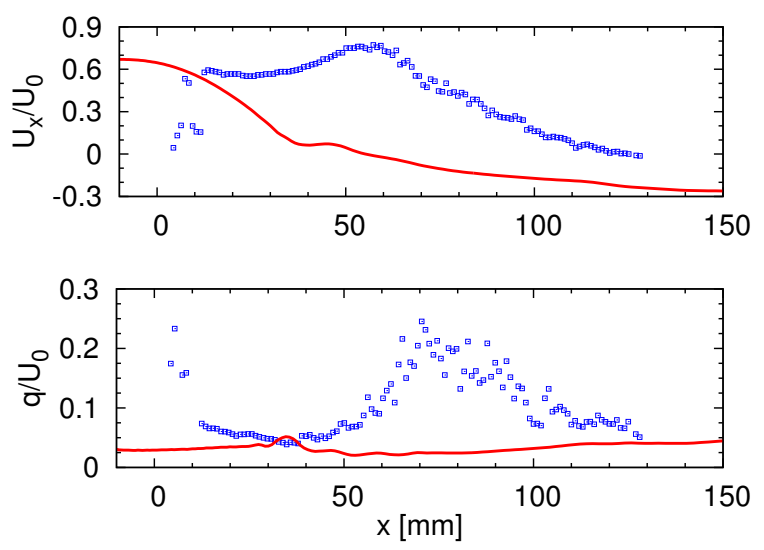

Figure 19: Comparison of mean axial velocity (top) and 2D velocity fluctuations $(q=$ $\sqrt{u_{x}^{\prime 2}+u_{r}^{\prime 2}}$ - bottom) for the hydrogen/air flame with experiments (symbols) along the centerline. LES results with unity Lewis number assumption are shown.

flamelet parameters, is presumed to depend on the corresponding filtered quantity and subfilter variances.

In LES, the scalar transport equations for the filtered progress variable and other flamelet parameters are solved to obtained the $\mathrm{PDF}, \widetilde{P}(\mathbf{S})$. The equation for the progress variable is given as

$$
\frac{\partial \bar{\rho} \widetilde{C}}{\partial t}+\frac{\partial}{\partial x_{i}}\left(\bar{\rho} \widetilde{u}_{i} \widetilde{C}\right)=\frac{\partial}{\partial x_{i}}\left(\bar{\rho}\left(\widetilde{D}+D_{t}\right) \frac{\partial \widetilde{C}}{\partial x_{i}}\right)+\overline{\dot{\omega}_{C}}
$$

where the filtered source term for the progress variable $\left(\overline{\dot{\omega}_{C}}\right)$ is evaluated using Eq. 35. The source term for progress variable is coupled with the filtered level set function, $\hat{G}$, in a manner similar to the coupled approach introduced by Moureau et al. [13].

For methane combustion, most of the major chemical species are characterized by a very similar diffusion coefficient, and the assumption of unity Lewis number for all species is typically justified. Following this assumption, the steady flamelet equations are derived, and 

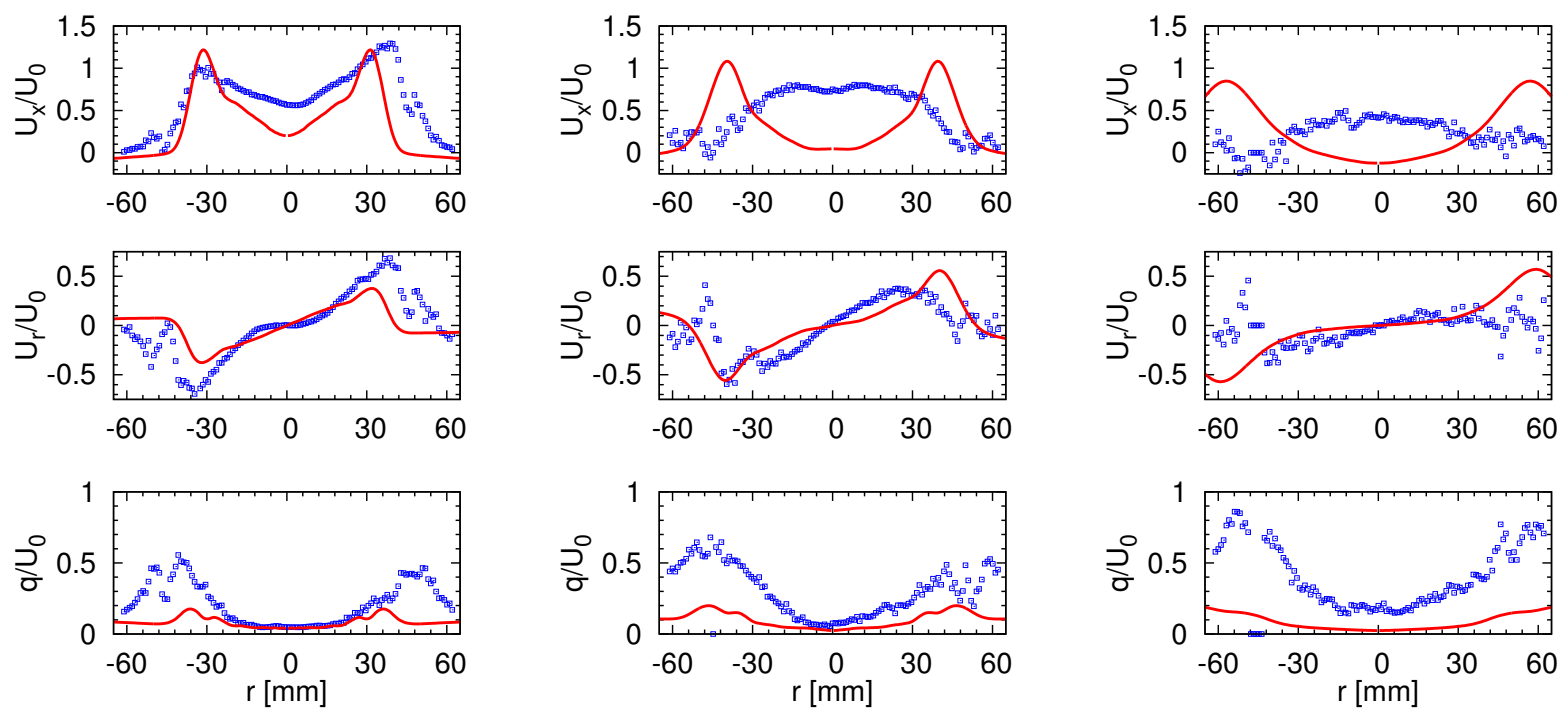

(a) $x=30 \mathrm{~mm}$

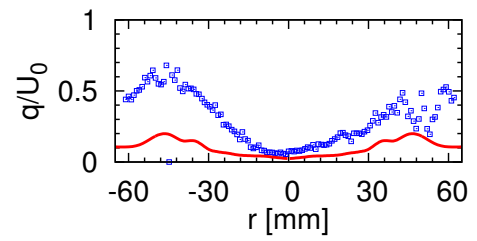

(b) $x=50 \mathrm{~mm}$

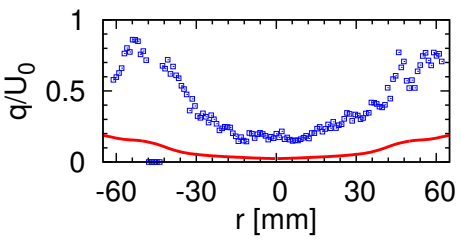

(c) $x=80 \mathrm{~mm}$

Figure 20: Comparison of mean axial velocity (top), mean radial velocity (center), and 2D velocity fluctuations $\left(q=\sqrt{u_{x}^{\prime 2}+u_{r}^{\prime 2}}\right.$ - bottom) for the hydrogen/air flame with experiments (symbols) at three locations downstream of the injection. LES results with unity Lewis number assumption are shown.

the various mixture properties and source terms are expressed as a function of a unique scalar.

In the case of hydrogen combustion, the Lewis number of the fuel $\left(\mathrm{H}_{2}\right)$ is much smaller than unity, typically around 0.3. In premixed flames, hydrogen molecules diffuse faster than temperature and the other species, ultimately leading to strong local mixture inhomogeneities. These regions of leaner or richer mixtures will then affect the laminar burning velocities, which is key to modeling of turbulent premixed flames in LES.

\subsection{Mixture Fraction/Progress Variable Approach for Lean Hy- drogen Premixed Combustion}

In this work, a new combustion model based on two independent transported scalars is developed to accurately account for differential diffusion effects of hydrogen molecules. This model is a direct extension of the methodology presented in the previous sections and relies on the mixture fraction $(Z)$ and the progress variable $(c)$ to describe the mixture composition. To account for the very different Lewis number of the fuel, the equation for mixture fraction 

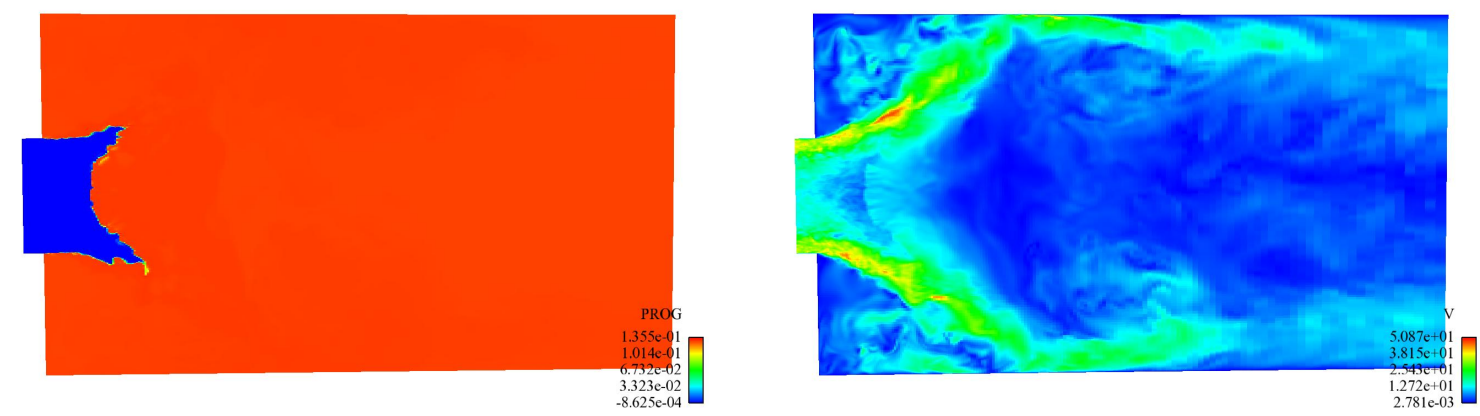

Figure 21: Instantaneous fields of the progress variable (left) and velocity (right) for the simulation of the flash-back in the lean hydrogen premixed low-swirl injector $(\phi=0.5)$. LES results with the unity Lewis number assumption are shown.
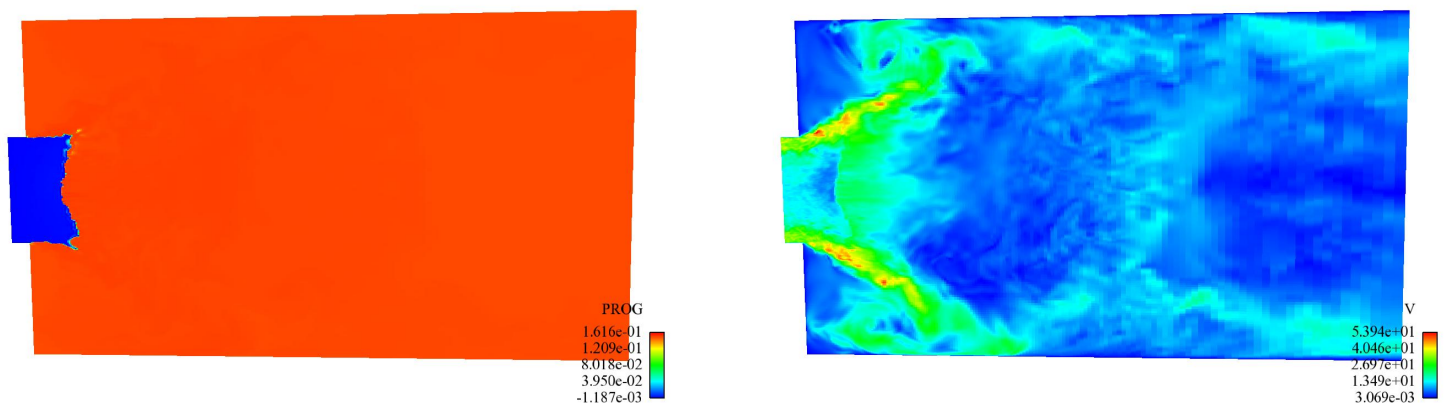

Figure 22: Instantaneous fields of the progress variable (left) and velocity (right) for the simulation of the flash-back in the lean hydrogen premixed low-swirl injector $(\phi=0.6)$. LES results with the unity Lewis number assumption are shown. 
is transformed. In a simulation, two scalar transport equations have to be solved,

$$
\begin{aligned}
\frac{\partial \rho Z}{\partial t}+\frac{\partial}{\partial x_{i}}\left(\rho u_{i} Z\right) & =\frac{\partial}{\partial x_{i}}\left(\rho D \frac{\partial Z}{\partial x_{i}}\right)+\dot{\omega}_{Z} \\
\frac{\partial \rho c}{\partial t}+\frac{\partial}{\partial x_{i}}\left(\rho u_{i} c\right) & =\frac{\partial}{\partial x_{i}}\left(\rho D \frac{\partial c}{\partial x_{i}}\right)+\dot{\omega}_{c} .
\end{aligned}
$$

The only difference between the present model and the model presented in the previous section resides in the additional source term for mixture fraction, mixture fraction is not anymore a conserved scalar. The full expression of the source term will be presented in the following subsection.

Then, any arbitrary reactive variable, including the source terms $\dot{\omega}_{c}$ and $\dot{\omega}_{S}$, can be evaluated as a function of these two independent variables. As presented in the previous section, two tables $\left(\phi_{i}(Z, c)\right)$ are used in order to accurately describe partially premixed combustion. In addition, a level set equation is solved to correctly predict the source term $\left(\dot{\omega}_{c}\right)$ in the limit of premixed combustion

$$
\frac{\partial \mathcal{G}}{\partial t}+u_{j} \frac{\partial \mathcal{G}}{\partial x_{j}}=\mathcal{D} \kappa|\nabla \mathcal{G}|+S_{L, u}|\nabla \mathcal{G}|,
$$

where $\kappa$ is the local flame curvature, and $S_{L, u}$ the laminar burning velocity.

For the limit of premixed combustion, a series of laminar premixed flames are simulated with a detailed chemical model. Laminar flat flames are considered as examples of unstretched flames. Then, the solutions of these flames are used to create a premixed table $\left(\phi_{i}(Z, c)\right)$ of all relevant properties, including the sources terms $\left(\dot{\omega}_{c}\right.$ and $\left.\dot{\omega}_{Z}\right)$. Finally, a third table is created to describe the dependence of the laminar burning velocity $\left(S_{L, u}\right)$ upon variations in mixture fraction $(Z)$.

\subsubsection{Modeling Assumptions}

In the case of non-unity Lewis number combustion, the mixture fraction is not anymore a conserved scalar and an additional source term $\left(\dot{\omega}_{Z}\right)$ appears in the transport equation. To derive this term, we consider the following assumptions:

- one-step irreversible chemical reaction

$$
\nu_{F} F+\nu_{O} O_{2} \rightarrow \nu_{P} P
$$

- fuel has a Lewis number different than unity $(L e \neq 1)$

- all other species have a unity Lewis number

Figure 23 shows the fields of mixture fraction (its exact definition will be given later) and progress variable across a lean hydrogen premixed flame $(\phi=0.3)$. This flame was simulated with the FlameMaster program [27] with detailed chemistry [25] and the Lewis number of all 


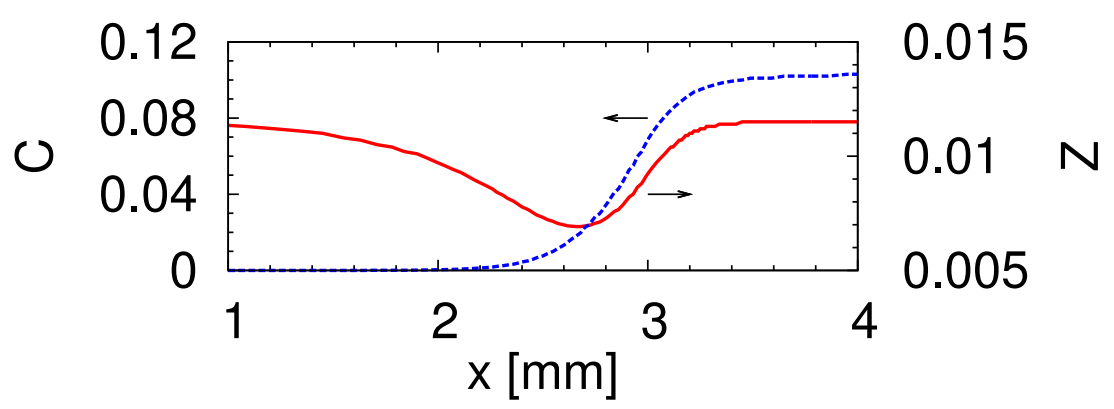

Figure 23: Mixture fraction fluctuations and progress variable across a lean hydrogen premixed flame $(\phi=0.4)$.

species but hydrogen set to unity $\left(L e_{H_{2}}=0.3\right)$. As expected, the progress variable defined as the mass fraction of water $\left(\mathrm{H}_{2} \mathrm{O}\right)$ increases in the flame. On the other hand, as a result of preferential diffusion of hydrogen, mixture fraction is not constant. It decreases near the flame front as hydrogen is transported towards the burnt side of the flame.

\subsubsection{Diffusion Flux}

As the local fluctuations in mixture fraction are due to differential diffusion, we start the analysis from the diffusion fluxes

$$
\mathbf{j}_{i}=\rho Y_{i} \mathbf{V}_{i}=-\rho D_{i} \frac{Y_{i}}{X_{i}} \nabla X_{i}+\rho Y_{i} \mathbf{V}_{c},
$$

where the velocity correction $\mathbf{V}_{c}$ is introduced to ensure that the net sum of all diffusion fluxes is null $\left(\sum \mathbf{j}_{i}=0\right)$. Following the assumptions made about the Lewis numbers of the different species, we can express the velocity correction as

$$
\mathbf{V}_{c}=D\left(\frac{1}{L e}-1\right) \nabla Y_{F}+D\left(1-Y_{F}+\frac{Y_{F}}{L e}\right) \frac{\nabla W}{W},
$$

where $D$ is the diffusivity of all species but the fuel, and $W$ is the molecular weight. Similarly, we can express the diffusion fluxes for the fuel and the oxidizer as

$$
\begin{aligned}
\mathbf{j}_{F}= & -\rho D\left(\frac{1-Y_{F}}{L e}+Y_{F}\right) \nabla Y_{F} \\
& +Y_{F}\left(1-Y_{F}\right) \rho D\left(1-\frac{1}{L e}\right) \frac{\nabla W}{W}
\end{aligned}
$$

and

$$
\begin{aligned}
\mathbf{j}_{O_{2}}= & -\rho D \nabla Y_{O_{2}}+\rho D Y_{O_{2}}\left(\frac{1}{L e}-1\right) \nabla Y_{F} \\
& +Y_{F} Y_{O_{2}} D\left(\frac{1}{L e}-1\right) \frac{\nabla W}{W} .
\end{aligned}
$$


For a one-step irreversible chemical reaction, it is convenient to define the mixture fraction (Z) as

$$
Z=\frac{\nu Y_{F}-Y_{O_{2}}+Y_{O_{2}, 2}}{\nu+Y_{O_{2}, 2}},
$$

where $Y_{O_{2}, 2}$ is the mass fraction of oxidizer in air. Following this definition, the transport equation for this mixture fraction is free of chemical source terms. However, contrarily to combustion with unity Lewis number fuel, the diffusion term is not only a function of mixture fraction. Combining Eqs. 42, 43, and 44, we can evaluate the diffusion flux for mixture fraction as

$$
\begin{aligned}
\mathbf{j}_{Z}= & -\rho D \nabla Z \\
& -\rho D\left(\frac{1}{L e}-1\right)(1-Z) \nabla Y_{F} \\
& +\rho D Y_{F}\left(\frac{1}{L e}-1\right)(1-Z) \frac{\nabla W}{W} .
\end{aligned}
$$

The first term corresponds to the standard molecular diffusion of mixture fraction. The second term corresponds to differential diffusion due to non unity Lewis number fuel. Finally, the third term corresponds to diffusion due to differences in molecular weight. In the case of unity Lewis number fuel, the second and third terms disappear, and we recover the standard molecular diffusion.

Figure 24 shows the relative importance of the different terms for the mixture fraction flux for the same lean hydrogen premixed flame as shown in Fig. 23. As expected, due to local fluctuations in mixture fraction, the molecular diffusion term is not zero and alternates in sign. On the other hand, the second term corresponding to the effects of differential diffusion remains large and positive indicating a transport of mixture fraction towards larger progress variable. This result is consistent with the observations made on Fig. 23. Finally, the third term corresponding to the effects of varying molecular weight remains small and thus will be neglected in the rest of the work.

The model for hydrogen combustion is not complete as the hydrogen mass fraction appears in Eq. 45 and should be expressed as a function of mixture fraction and progress variable. When considering a one-step irreversible chemical reaction, the fuel mass fraction can be expressed easily as

$$
Y_{F}=Z-C \cdot \frac{W_{F}}{W_{C}},
$$

where $W_{C}$ is the molecular weight of the products. In deriving the above expression, dilution effects due to an increase in the total number of moles was neglected.

\subsubsection{Premixed Chemical Table}

As in the case of partially premixed combustion, all relevant chemical properties such as temperature, density, burning velocity, and source terms are mapped onto mixture fraction 


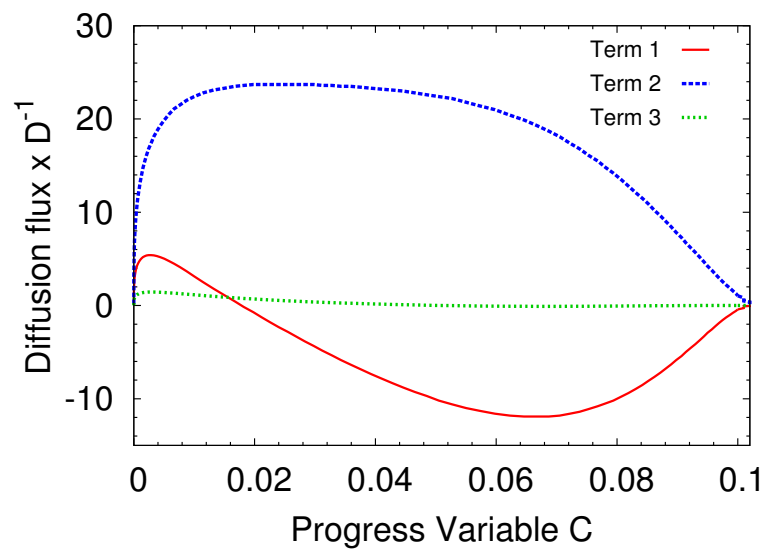

Figure 24: Relative magnitude of the different terms in the mixture fraction diffusion flux (Eq. 45) for a lean hydrogen premixed flame $(\phi=0.4)$.

and progress variable as $\phi=\phi(Z, C)$. The success of the modeling approach resides strongly on the ability of the mapping to describe properly all chemical phase state. Figure 25 shows the fluctuations of progress variable and mixture fraction in several 1D laminar premixed hydrogen flames with different overall equivalence ratios. As expected, for larger equivalence ratios, the average mixture fraction and maximum progress variable are larger. More important, no point in the Z-C space is described by two different flamelets. This unique mapping is a critical requirement for the definition of a chemical table.

\subsubsection{Validation in Laminar Hydrogen Flames}

As a first step towards non-unity Lewis number fuels, we validate the mixture fraction/progress variable approach in the simulation of 1D freely propagating flames of methane and hydrogen. Here, we show only the results for the hydrogen flame with an equivalence ratio of $\phi=0.4$. The simulations were performed on a $1 \mathrm{D}$ grid of length $10 \mathrm{~mm}$ with 100 grid points. The convective CFL condition was around 0.35. The Lewis number of all species but hydrogen was fixed at unity $\left(L e_{H_{2}}=0.3\right)$ as was done for Figs. 23 and 24 .

Figure 26 shows a comparison of the results for the simulation of the hydrogen flame with the Z-C model presented before and detailed chemistry. As expected, the flame position increases linearly with time characteristic of a constant flame speed. The evaluation of the flame speed at each location shows significant fluctuations around the theoretical value $\left(S_{L}=20.14 \mathrm{~cm} / \mathrm{s}\right)$. Similar fluctuations can be observed for the evaluation of the flame thickness. However, these fluctuations are only a result of the numerical discretization of the temperature profile on the coarse grid. 


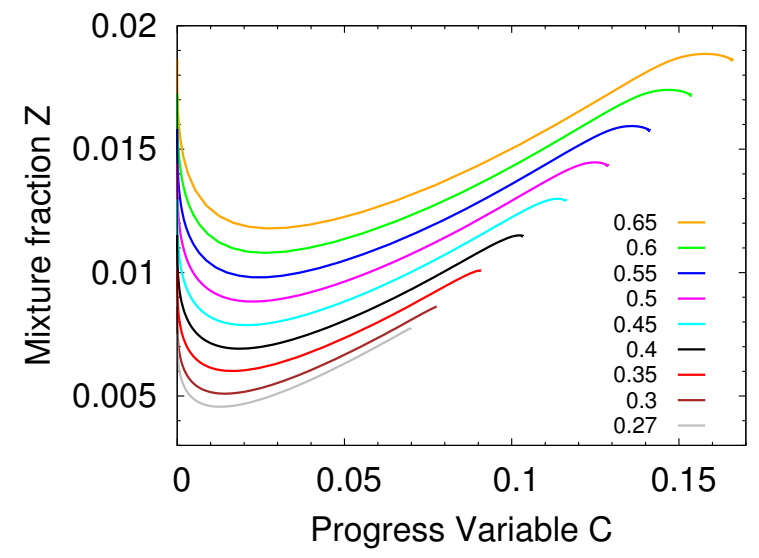

Figure 25: Progress variable and mixture fraction fluctuations along several hydrogen/air flamelets with different overall equivalence ratios.

\subsection{Hydrogen/Progress Variable Approach for Lean Hydrogen Pre- mixed Combustion}

In lean hydrogen premixed flames, the diffusion of the deficient fuel, i.e. hydrogen, is much faster than thermal conduction. The diffusive-thermal instability can therefore play an important role in flame propagation. Because the rate of hydrogen diffusion is very different from that of thermal conduction, the local flame structure is not uniquely described by using the reaction progress variable only. In the hydrogen/progress variable approach, the chemical states are approximated using the progress variable and hydrogen: $\mathbf{S}=\left(C, Y_{\mathrm{H}_{2}}\right)$. The flamelet library is generated by varying the equivalence ratio of unstretched laminar flames.

\section{Validation Using Direct Numerical Simulation Data for Lean Hydrogen Combustion}

DNS of a freely propagating lean hydrogen premixed flame is performed to investigate the diffusive-thermal instability under conditions relevant to low swirl flames investigated in Cheng et al. [26]. The ultimate objective is to answer the question if the models discussed in Sec. 6 can capture the salient features caused by the diffusive-thermal instability in lean hydrogen flames.

As a first step, 2-D DNS with uniform inflow velocity is performed. The NGA code is used to solve low Mach number equations and the stiffness in a detailed chemical mechanism is dealt with a stiff ODE solver, DASSL. At the left boundary, premixed fuel/air mixture is injected at an uniform velocity. The periodic boundary condition is applied for upper and lower boundaries. The computational domain is $10 \mathrm{~cm} \times 10 \mathrm{~cm}$, which is discretized into $1024 \times 1024$ grid points. A detailed chemical mechanism consisting of 9 species and 19 


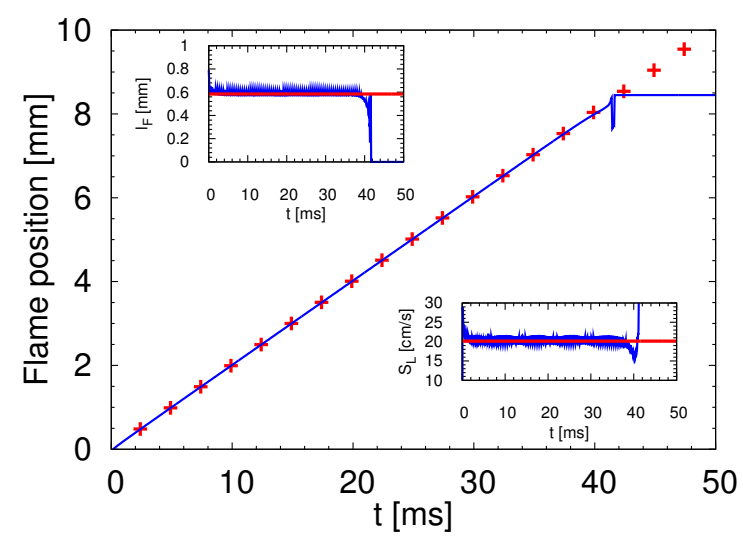

Figure 26: Flame position (center), flame thickness (top left), and flame speed (bottom right) for a $1 \mathrm{D}$ freely propagating premixed hydrogen flame $(\phi=0.4)$ with detailed chemistry (red) and the Z-C model (blue).

reactions is employed. During the computation, inflow velocity is adjusted to maintain flame fronts at the center of the computational domain. The initial flame location $x_{f, 0}$ is specified according to

$$
x_{f, 0}(y)=\sum_{i} A_{i} \cos \left(\frac{2 \pi y}{\lambda_{i}}\right),
$$

where $A_{i}$ and $\lambda_{i}$ are the amplitude and the wavelength of the $i$-th perturbation. A solution of the unstretched laminar flame is used to define initial fields of species mass fractions and temperature. This configuration has been studied by Kodowaki et al. [28] and Yuan et al. [29], but with a single-step chemistry.

Figures 27-29 show instantaneous fields of temperature and mass fractions of $\mathrm{H}, \mathrm{OH}$, and $\mathrm{H}_{2}$ at different times. Initially, perturbations with a short wave length grow fast. When the flame front is concave toward the unburned mixture, radical concentrations are negligible and local extinction of the flame is observed. At a later time, the cells merge and form bigger cells. Small cells are then recreated along the surfaces of large cells. This nonlinear stage of the cell evolution is similar to that observed in [28] and [29].

Figure 30 shows instantaneous fields of $\mathrm{H}_{2} \mathrm{O}$ at different times. Initial conditions are different from those for Figs. 27-29. The same flame as in Fig. 30 is simulated using the two-scalar (hydrogen/progress-variable) model, and results for different resolutions are shown in Figs. 31-33. The hydrogen and progress variable are used as flamelet parameters. For $1024 \times 1024$ grid points, overall flame shapes are similar to those in DNS, while details are different. The flame shapes remain similar for a coarse mesh, $512 \times 512$. A significant change in flame characteristics is, however, observed for $256 \times 256$ grid points. This suggests that the prediction of the diffusive-thermal instability can be sensitive to the resolution of the scalar fields and that a more elaborate approach, such as coupling with the level-set method, should be employed for LES. In future, we will investigate this issue in detail.

Figure 34 shows the consumption speed of reaction fronts as a function of the flame 

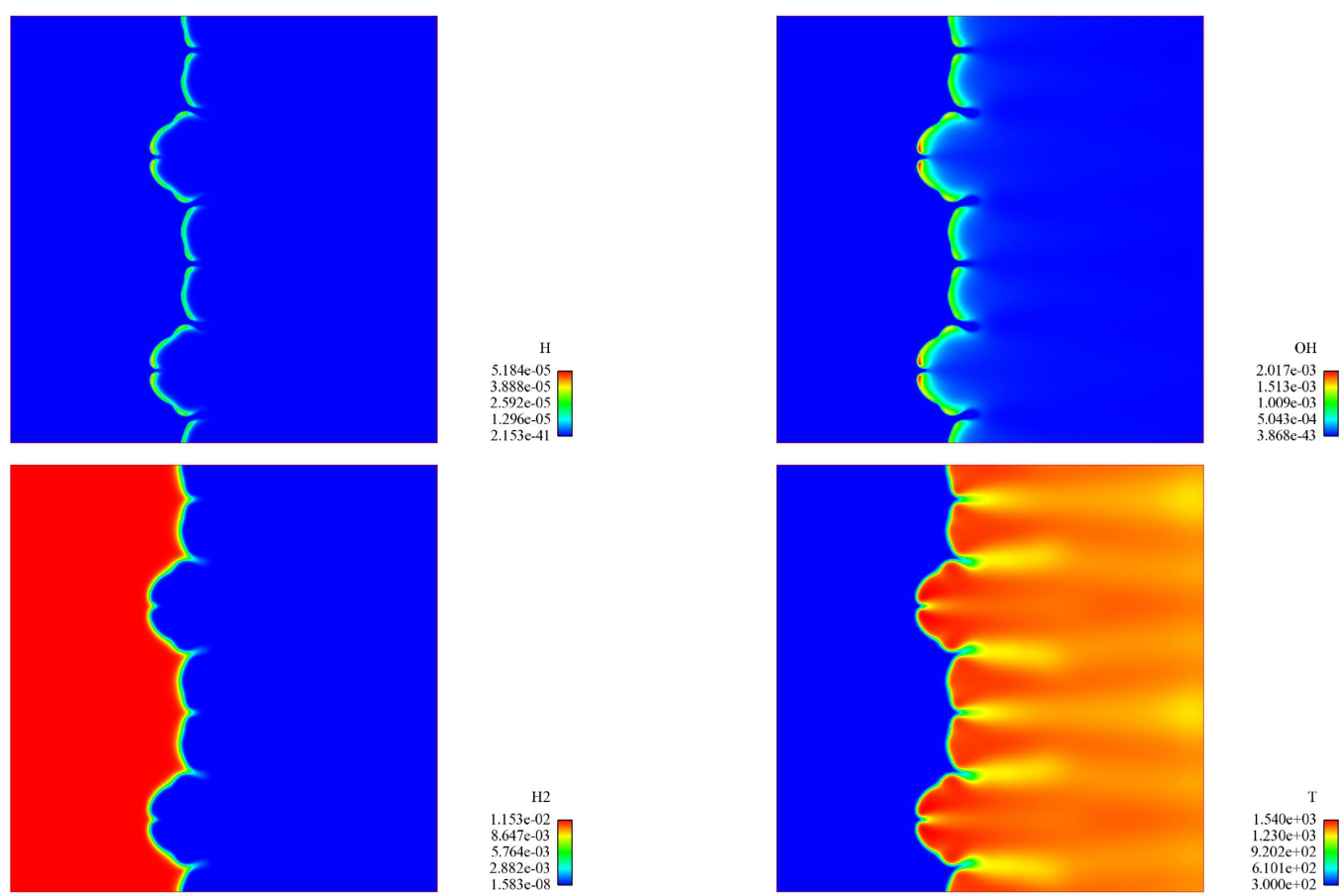

$3.868 \mathrm{e}-43$

Figure 27: Instantaneous fields of $\mathrm{H}$ (upper left), $\mathrm{OH}$ (upper right), $\mathrm{H}_{2}$ (lower left) and temperature (lower right) for a freely propagating lean hydrogen premixed flame at $t=0.06 \mathrm{~s}$.
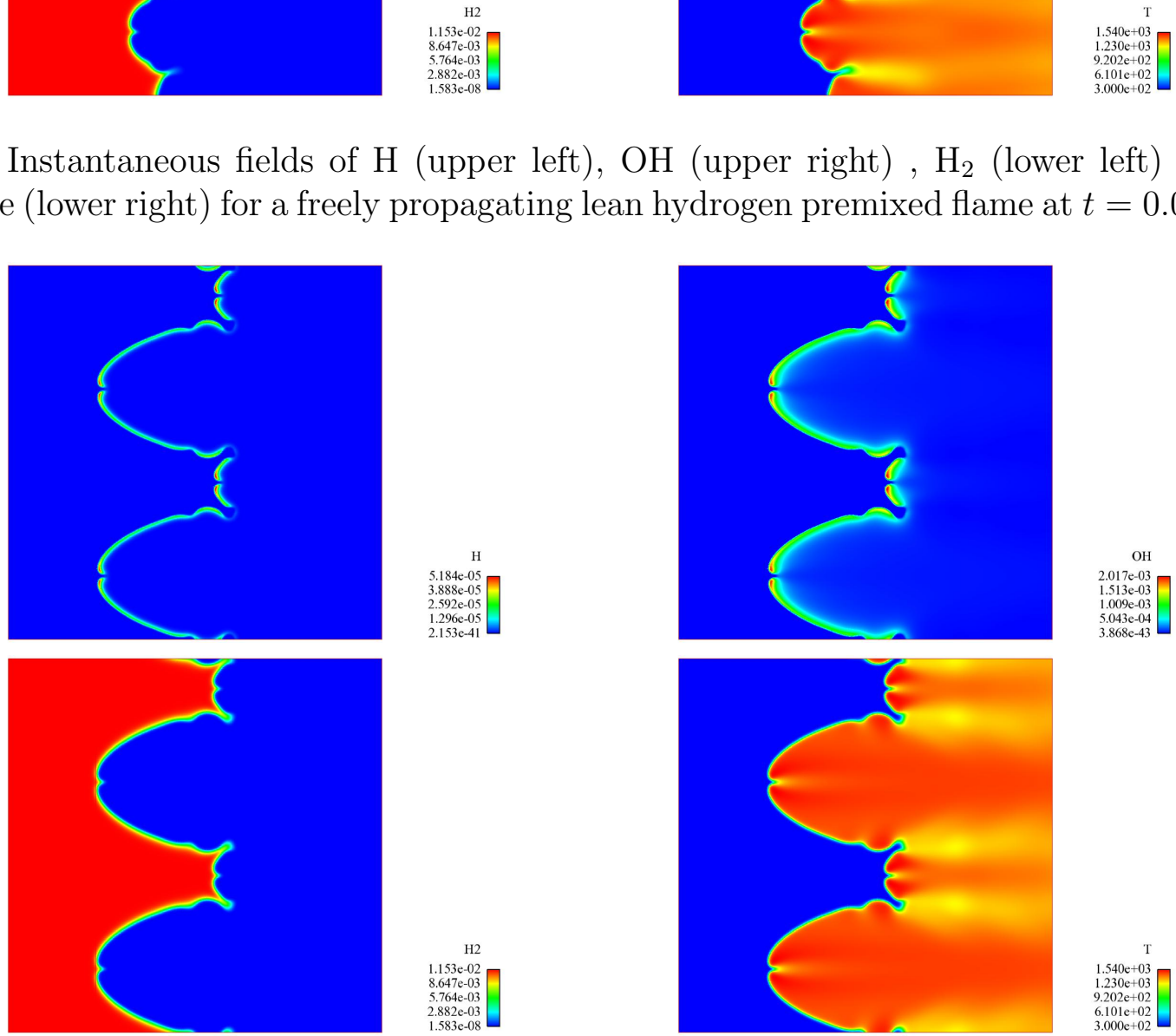

Figure 28: Instantaneous fields of $\mathrm{H}$ (upper left), $\mathrm{OH}$ (upper right), $\mathrm{H}_{2}$ (lower left) and temperature (lower right) for a freely propagating lean hydrogen premixed flame at $t=0.11 \mathrm{~s}$. 

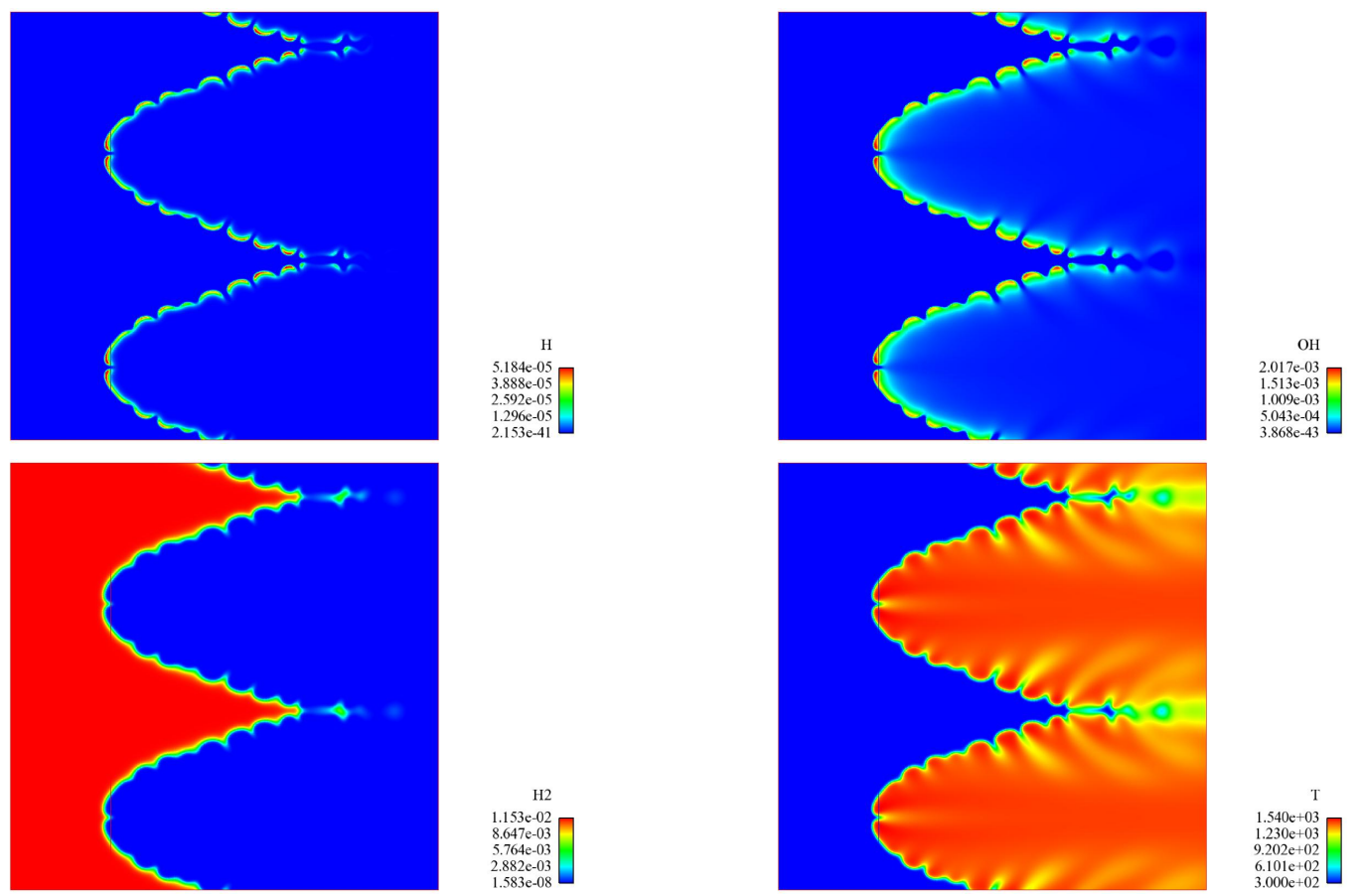

Figure 29: Instantaneous fields of $\mathrm{H}$ (upper left), $\mathrm{OH}$ (upper right), $\mathrm{H}_{2}$ (lower left) and temperature (lower right) for a freely propagating lean hydrogen premixed flame at $t=0.16 \mathrm{~s}$.

curvature. A key question regarding the modeling of the diffusive-thermal instability in lean hydrogen premixed flames is if a model captures the dependence of the propagation speed of reaction fronts on the curvature of the fronts. In order to answer that question, the consumption speed of reaction fronts in DNS data is evaluated and compared with the propagation speed predicted using the two-scalar (hydrogen/progress-variable) model in Fig. 35. The consumption speed of a flame element is obtained integrating the reaction rate in a flame normal direction:

$$
S_{c}=\frac{1}{\rho Y_{\mathrm{H}_{2}, \mathrm{in}}} \int_{n_{-}}^{n_{+}} \omega_{\mathrm{H}_{2}} d n
$$

where $S_{c}$ is the consumption speed, $Y_{\mathrm{H}_{2} \text {,in }}$ is the hydrogen mass fraction at the inlet, and $n$ is the flame normal direction. The flame normal $\mathbf{n}$ is defined by

$$
\mathbf{n}=-\frac{\nabla Y_{\mathrm{H}_{2} \mathrm{O}}}{\left|\nabla Y_{\mathrm{H}_{2} \mathrm{O}}\right|}
$$

In general, the flame is curved and a weighting factor (or the Jacobian of the generalized flame coordinate) is used in the integration [30]. However, the flame location used in defining the propagation speed is not well defined, and it is acceptable to set the weighting fact to be unity. The results in Fig. 35 show that the two-scalar model captures the curvaturedependence of the local flame speed reasonably well. 

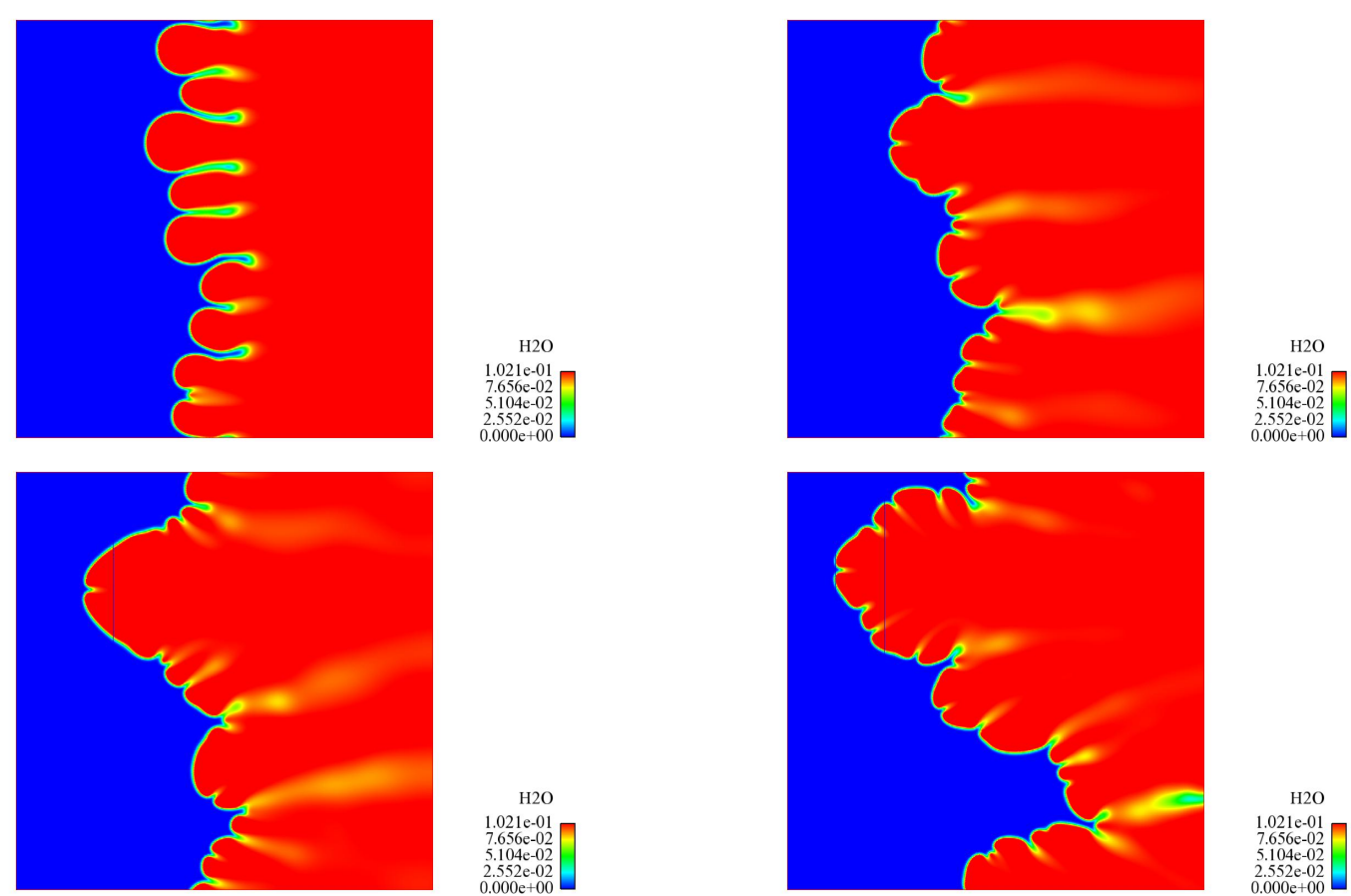

Figure 30: Instantaneous fields of $\mathrm{H}_{2} \mathrm{O}$ at $t=0.01 \mathrm{~s}$ (upper left), $t=0.06 \mathrm{~s}$ (upper right) ,$t=0.1 \mathrm{~s}$ (lower left) and $t=0.18 \mathrm{~s}$ (lower right) for DNS of a freely propagating lean hydrogen premixed flame. 

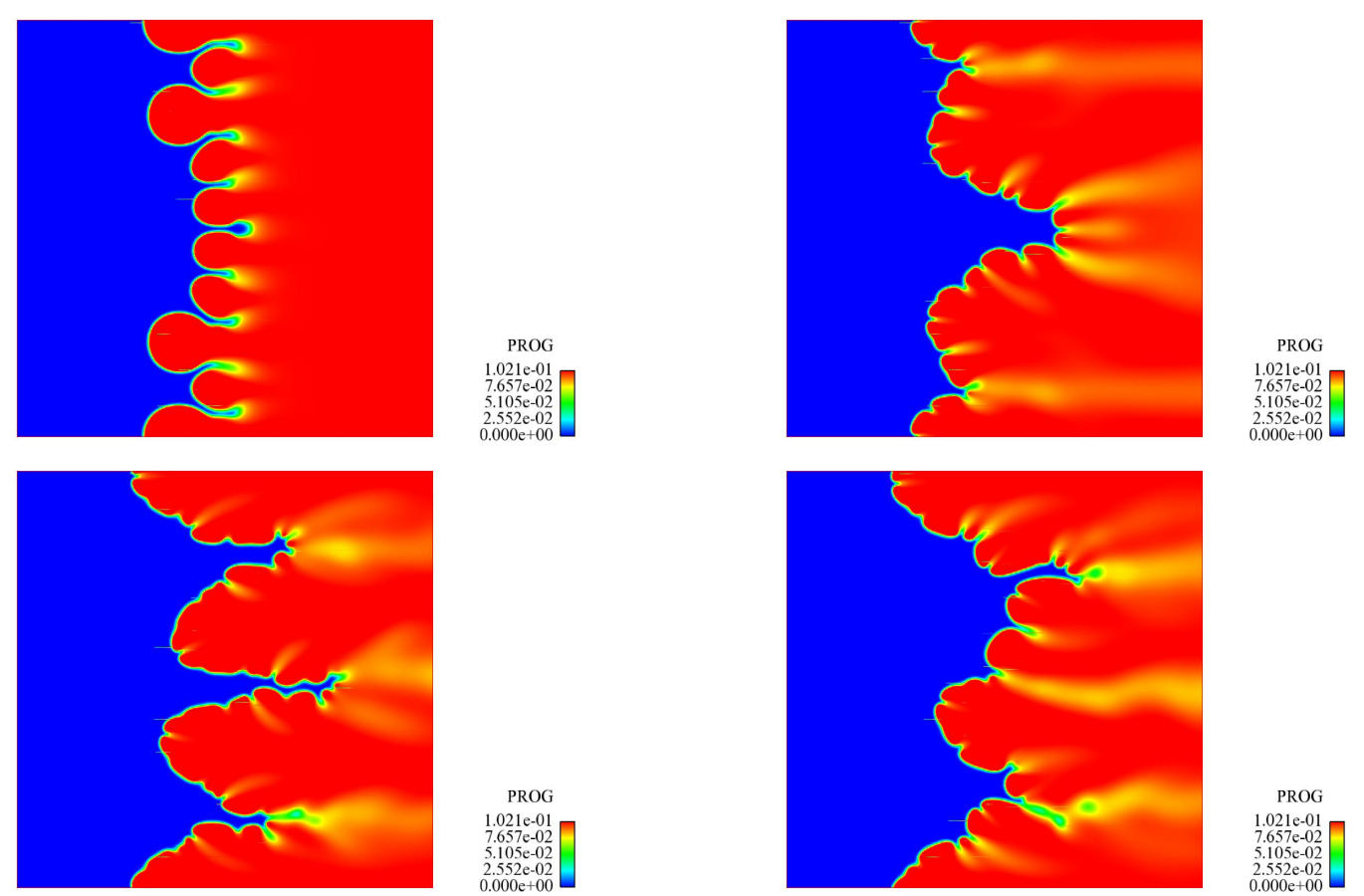

Figure 31: Instantaneous fields of $\mathrm{H}_{2} \mathrm{O}$ at $t=0.01 \mathrm{~s}$ (upper left), $t=0.06 \mathrm{~s}$ (upper right), $t=0.1 \mathrm{~s}$ (lower left) and $t=0.18 \mathrm{~s}$ (lower right) for the two-scalar model with $1024 \times 1024$ grid points. 

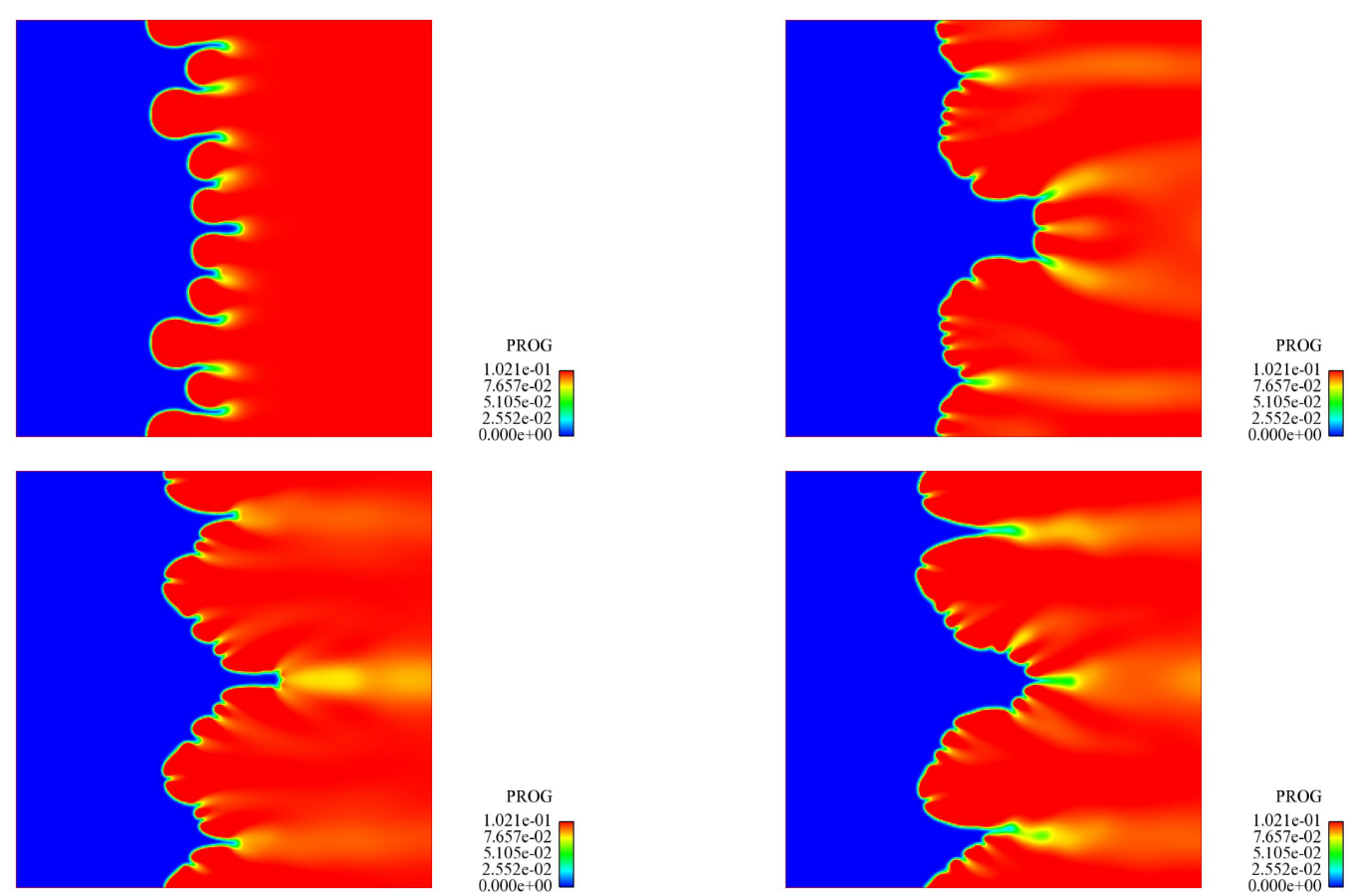

Figure 32: Instantaneous fields of $\mathrm{H}_{2} \mathrm{O}$ at $t=0.01 \mathrm{~s}$ (upper left), $t=0.06 \mathrm{~s}$ (upper right), $t=0.1 \mathrm{~s}$ (lower left) and $t=0.18 \mathrm{~s}$ (lower right) for the two-scalar model with $512 \times 512$ grid points. 

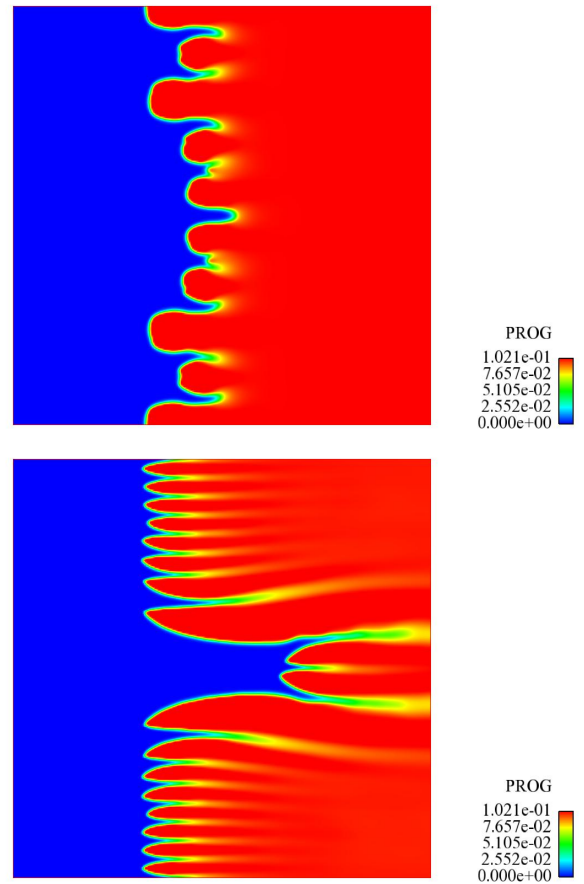
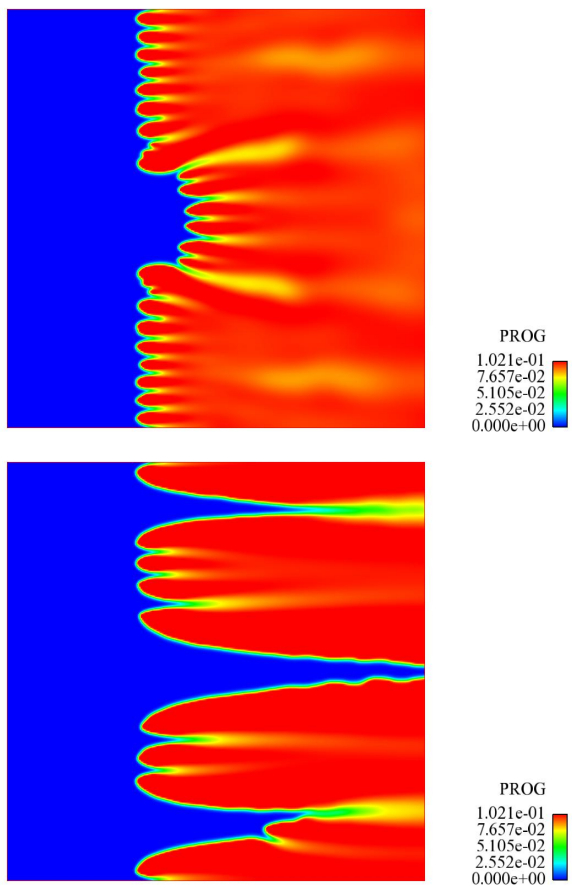

Figure 33: Instantaneous fields of $\mathrm{H}_{2} \mathrm{O}$ at $t=0.01 \mathrm{~s}$ (upper left), $t=0.06 \mathrm{~s}$ (upper right), $t=0.1 \mathrm{~s}$ (lower left) and $t=0.18 \mathrm{~s}$ (lower right) for the two-scalar model with $256 \times 256 \mathrm{grid}$ points.

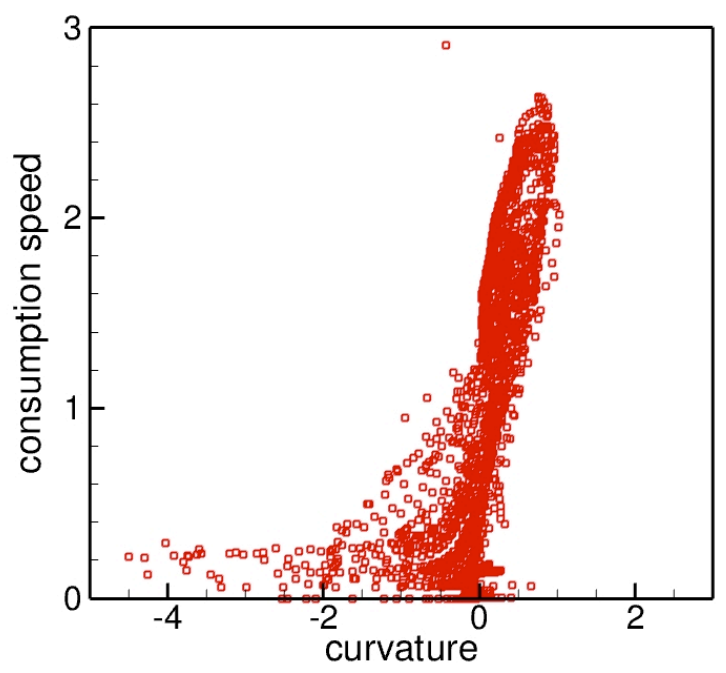

Figure 34: Correlation of the consumption speed with the flame curvature in DNS of a freely propagating lean hydrogen premixed flame. The consumption speed and the curvature are normalized by the propagation speed and thickness of the unstretched laminar flame, respectively. 


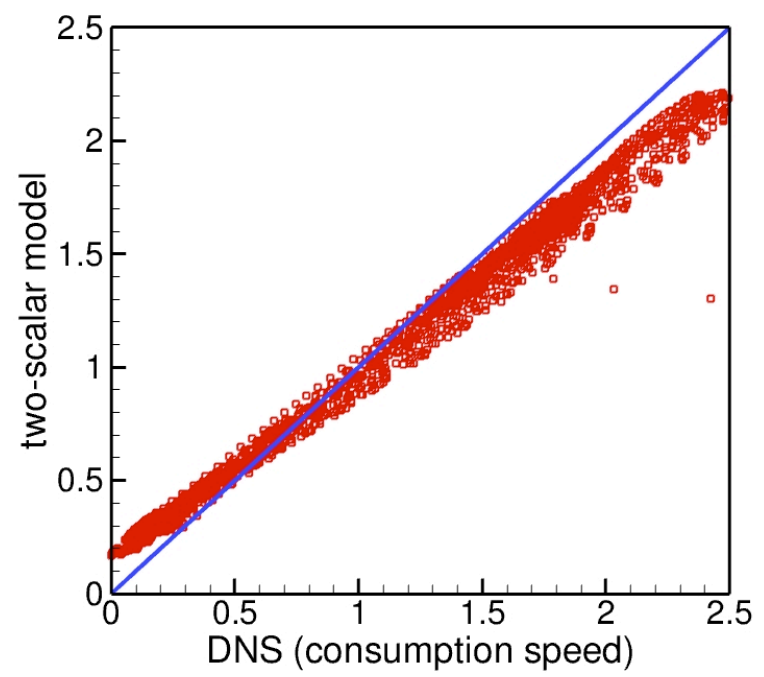

Figure 35: Comparison of the consumption speed from DNS of a freely propagating lean hydrogen premixed flame with the two-scalar model. The consumption speed is normalized by the unstretched laminar flame speed.

\section{Application to a Low-Swirl Hydrogen Flame}

The new model for describing non-unity Lewis number effects in LES is applied to a low swirl burner operated with an $\mathrm{H}_{2}$ /air mixture $(\phi=0.4)$. With confidence in the treatment of the boundary conditions and favorable predictions for methane combustion presented in Sec. 5, here, we focus on the effects of differential diffusion in a turbulent hydrogen flame. The experimental setup is the same as previously used for methane. The only differences reside in the fuel used $\left(\mathrm{H}_{2}\right)$ and the global equivalence ratio $(\phi=0.40)$.

\subsection{Large Eddy Simulation Using the Mixture-Fraction/Progress- Variable Approach}

Figure 36 shows a comparison of the mean axial velocity from the simulation and from the experiment. The experimental measurements of velocity away from the centerline suffer from large uncertainties. However, the comparison remains qualitatively good. According to Fig. 36, the downstream location of the $U=0$ iso-contour is predicted quite accurately by the simulation. A comparison of the hydrogen and methane LES results can be used to help understand the importance of the non-unity Lewis number model. Figures 15 and 36 demonstrate that some important differences exist between the methane and hydrogen flames. For instance, the mean hydrogen flame front (Fig. 36) is located much closer to the injection plane than the corresponding methane flame front (Fig. 15). Furthermore, the hydrogen simulation predicts an acceleration of the flow behind the flame front that is 


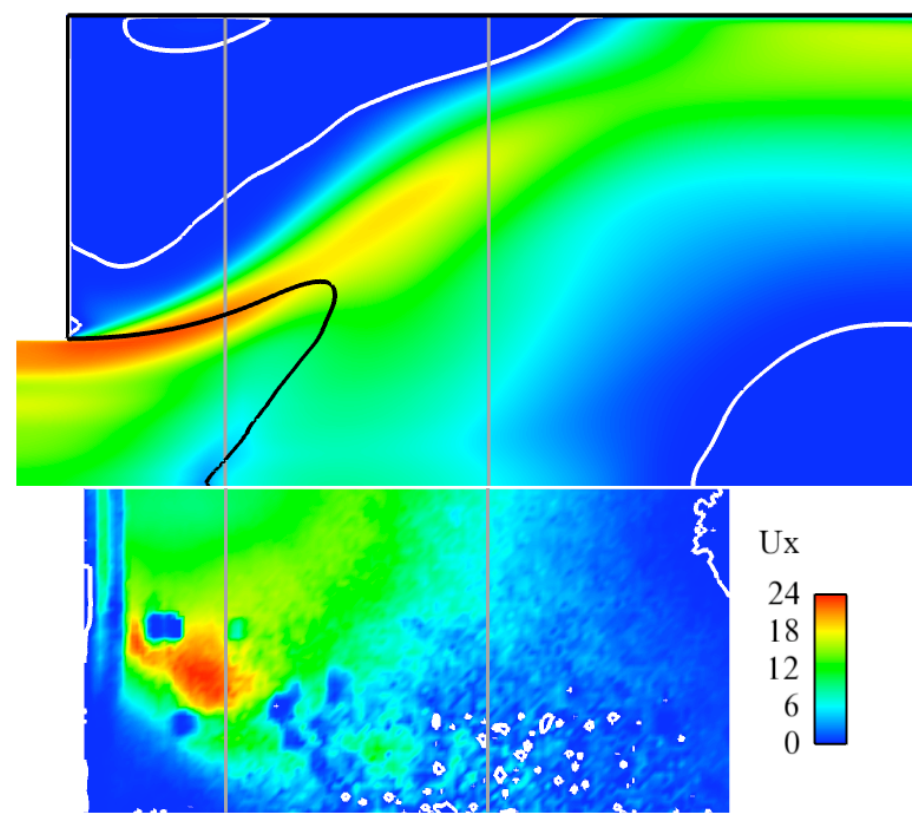

Figure 36: Comparison of mean axial velocity for the hydrogen flame from the simulation (top) and from the experiment (bottom). The recirculation regions are shown in white $\left(U_{x}=0\right.$ iso-contours). The mean flame location is shown in black $(C=0.055$ iso-contour $)$. The simulation was performed with the rescaled burning velocities.

consistent with the experiments. This strong acceleration was not present in the methane flame.

Figures 37 and 38 show a comparison of the experimental and the simulated mean and rms velocities. Three independent simulations were performed to analyze the effects of differential diffusion. The first simulation was performed assuming unity Lewis number (red dotted lines), the second simulation was performed with the model proposed in the previous section (blue dashed lines), and the third simulation was performed with the proposed model and with the source terms rescaled to match the experimental laminar burning velocities (green solid lines).

The experimental measurements suggest that the recirculation region is strongly affected by the use of a fuel with non-unity Lewis number. Relative to the simulation with unity Lewis number, the hydrogen simulations recirculation region is pushed farther downstream from $x \approx 50 \mathrm{~mm}$ to $x \approx 120 \mathrm{~mm}$. However, running the simulation with the same approach as for methane, i.e. without explicitly accounting for differential diffusion effects, predicts a strong and early recirculation region very similar to that found in the methane case. On the other hand, Figs. 37 and 38 demonstrates that the proposed treatment of non-unity Lewis numbers leads to a strong acceleration of the flow behind the flame front that is consistent with the experimental observations. This acceleration is visible in the mean and uctuating radial velocity plots around $x=30 \mathrm{~mm}$. The changing mixture fraction $(Z)$ values are the avenue through which the model for non-unity Lewis number acts. To illustrate these effects, 

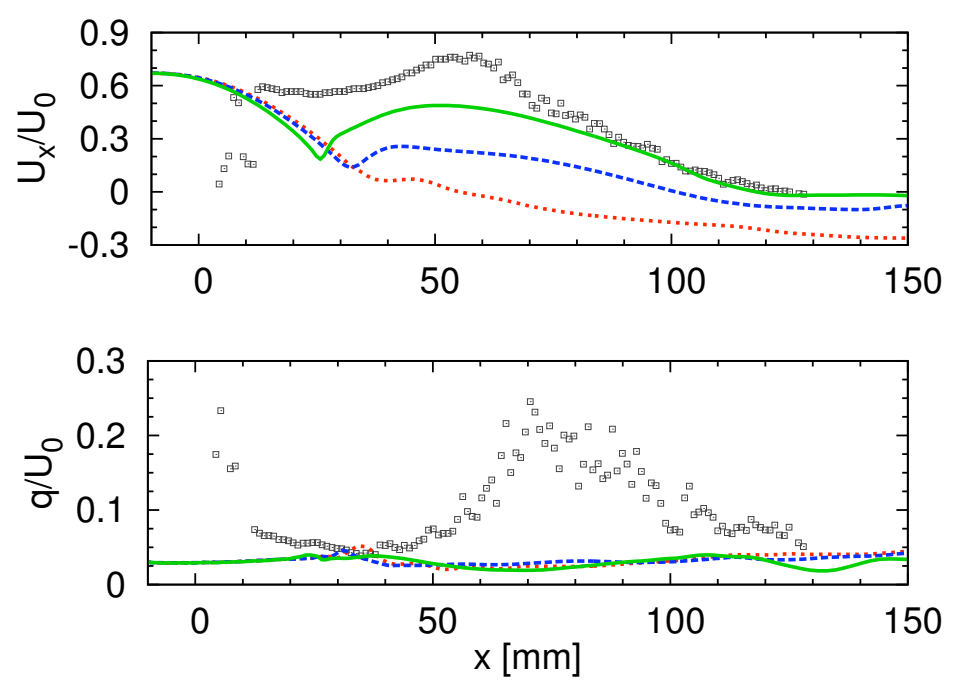

Figure 37: Comparison of mean axial velocity $\left(U_{x}\right)$ and $2 \mathrm{D}$ velocity fluctuations $(q=$ $\sqrt{u_{x}^{\prime 2}+u_{r}^{\prime 2}}$ ) for the hydrogen/air flame with experiments (symbols).

Fig. 39 shows an instantaneous profile of mixture fraction. When Lewis number effects are neglected, mixture fraction is expected to remain constant around $Z=0.011$. However, as a result of the additional source term due to differential diffusion, mixture fraction is not constant and fluctuates near the flame front. In regions of positive curvature (light color in Fig. 39), mixture fraction increases; in regions of negative curvature (dark colors), mixture fraction decreases. The chemistry tabulation associates higher (resp. lower) values of burning velocity and chemical source term for rich (resp. lean) mixtures. The difference of burning speed along the wrinkled flame front ultimately leads to the thermo-diffusive instabilities. From the numerical simulations, the effective burning speed of the mean flame front appears to be larger than without instabilities. This result is consistent with results from previous DNS of LBNL.

\subsection{Large Eddy Simulation Using the Hydrogen/Progress-Variable Approach}

To consider non-unity Lewis number effects, the lean hydrogen premixed flame is simulated using the hydrogen/progress variable approach. Figures 40 and 41 show the instantaneous fields of the progress variable and the velocity. In Fig. 41, the laminar flame speed is increased by a factor of three in order to investigate the effects of the laminar flame speed. The laminar flame speed predicted using a detailed chemical mechanism is typically smaller than the experimental data for very lean cases and the correction is made in order to match experimentally measured values. For $\phi=0.4$, the laminar flame speed is $0.15 \mathrm{~m} / \mathrm{s}$, while experimentally measured values are about $0.35 \mathrm{~m} / \mathrm{s}$. Note that the flame fronts in the low velocity region near the centerline are much more wrinkled that those in the high-velocity 

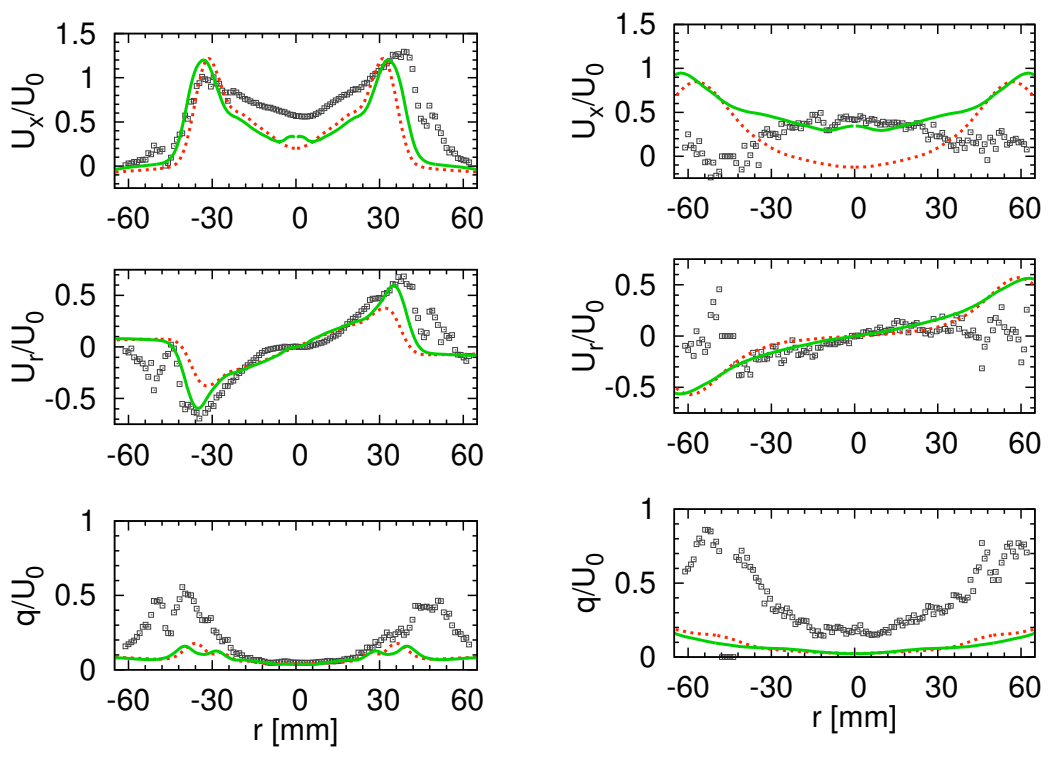

(a) $x=30 \mathrm{~mm}$

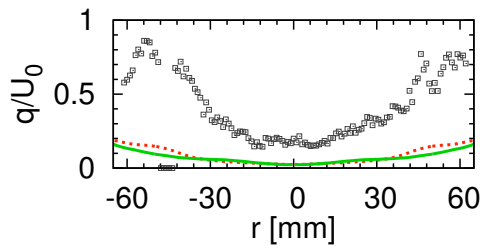

(b) $x=80 \mathrm{~mm}$

Figure 38: Comparison of mean axial velocity $\left(U_{x}\right)$, mean radial velocity $\left(U_{r}\right)$, and $2 \mathrm{D}$ velocity fluctuations $\left(q=\sqrt{u_{x}^{\prime 2}+u_{r}^{\prime 2}}\right)$ for the hydrogen/air flame with experiments (symbols) at three locations downstream of the injection.

region. The high degree of wrinkling is perhaps due to the diffusive-thermal instability, which is captured by the two-scalar model. Behind the flame fronts near the centerline, the increase of the velocity is observed, which is consistent with the experimental data. This increase in velocity is much more evident in the simulation with the increased laminar flame speed. Figure 42 shows the axial distribution of the mean axial velocity. Compared with the unity Lewis number solution in Figs. 44, the two-scalar model gives improved results. The improvement is more significant when the increased laminar flame speed is used. However, the increase in the axial velocity at $30 \mathrm{~mm}<x<50 \mathrm{~mm}$ is overpredicted by LES with the increased laminar flame speed. This may be partly because the artificial increase of the flame speed by a factor of three gives the laminar flame speed slightly higher than the experimentally measure value. The axial distribution of $2 \mathrm{D}$ velocity fluctuations is shown in Fig. 43. The two-scalar model with the increased laminar flame speed reproduces the increase of the turbulent intensity at $50 \mathrm{~mm}<x<100 \mathrm{~mm}$, while the predicted turbulent production is not as significant as in experiments. The radial distribution of velocity statistics are shown in Figs. 45-47. For these statistics, the two-scalar model does not perform very well. 


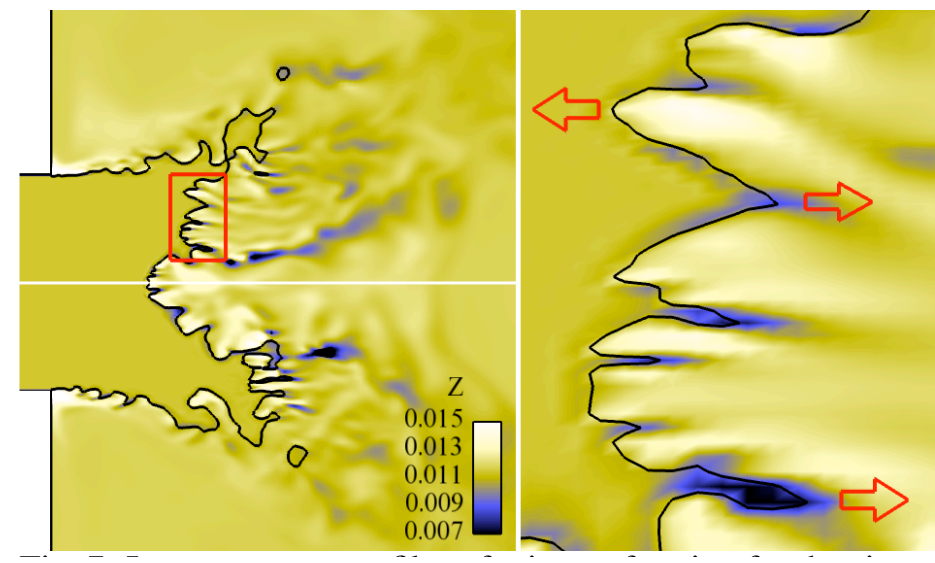

Figure 39: Comparison of mean axial velocity for the hydrogen flame from the simulation (top) and from the experiment (bottom). The recirculation regions are shown in white ( $U_{x}=0$ iso-contours). The mean flame location is shown in black ( $C=0.055$ iso-contour). The simulation was performed with the rescaled burning velocities.
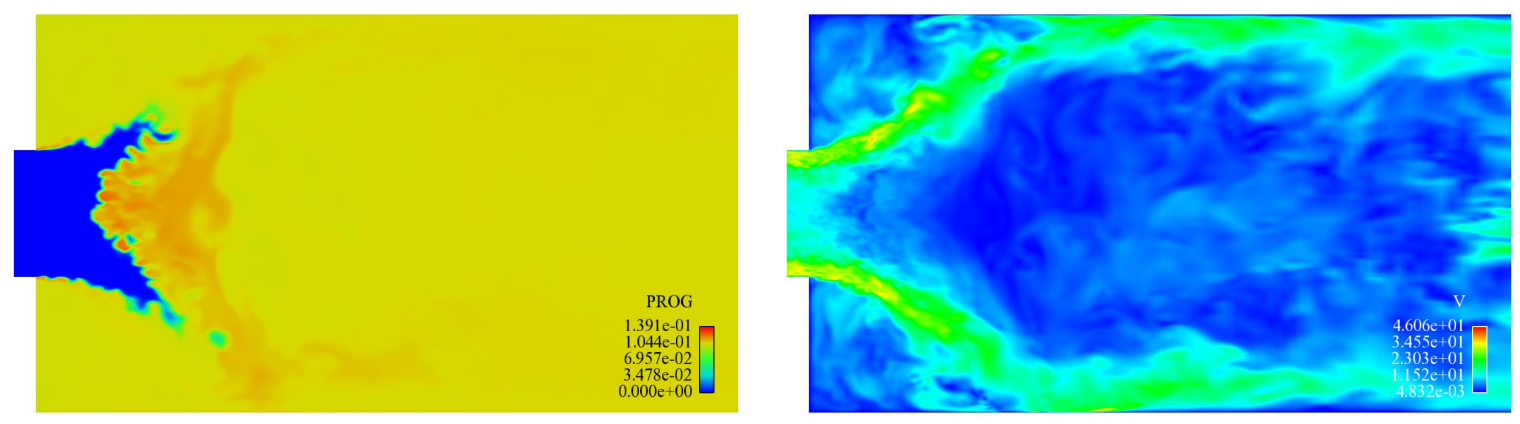

Figure 40: Instantaneous fields of the progress variable (left) and velocity (right) for the simulation of the lean hydrogen premixed low-swirl injector. LES is performed using the two-scalar model. 

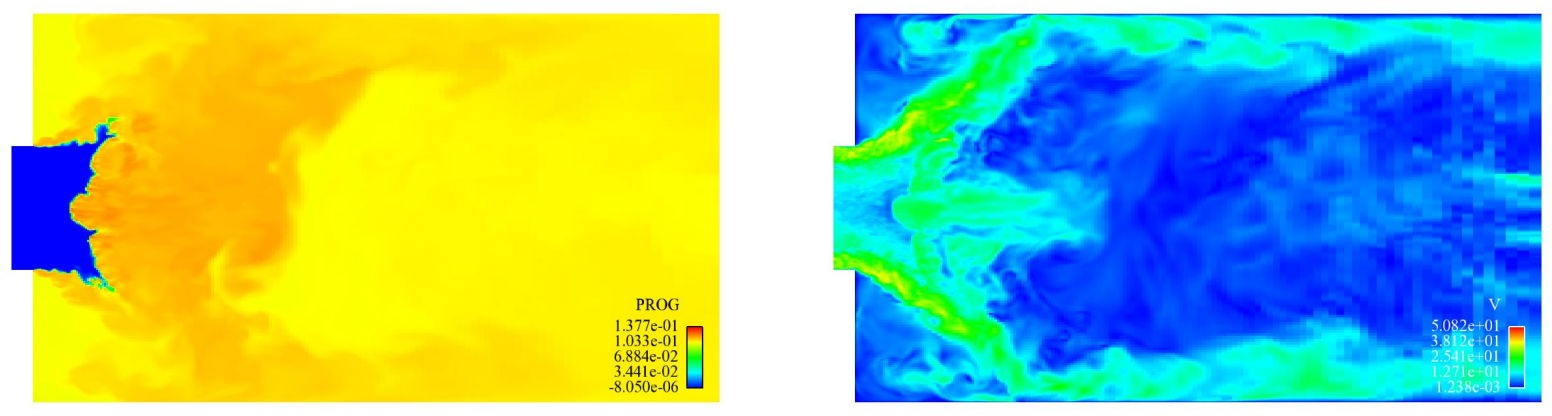

Figure 41: Instantaneous fields of the progress variable (left) and velocity (right) for the simulation of the lean hydrogen premixed low-swirl injector. LES is performed using the two-scalar model. The laminar flame speed is increased by a factor of three in order to compensate an error in calculation of the laminar flame speed for lean hydrogen premixed flames.
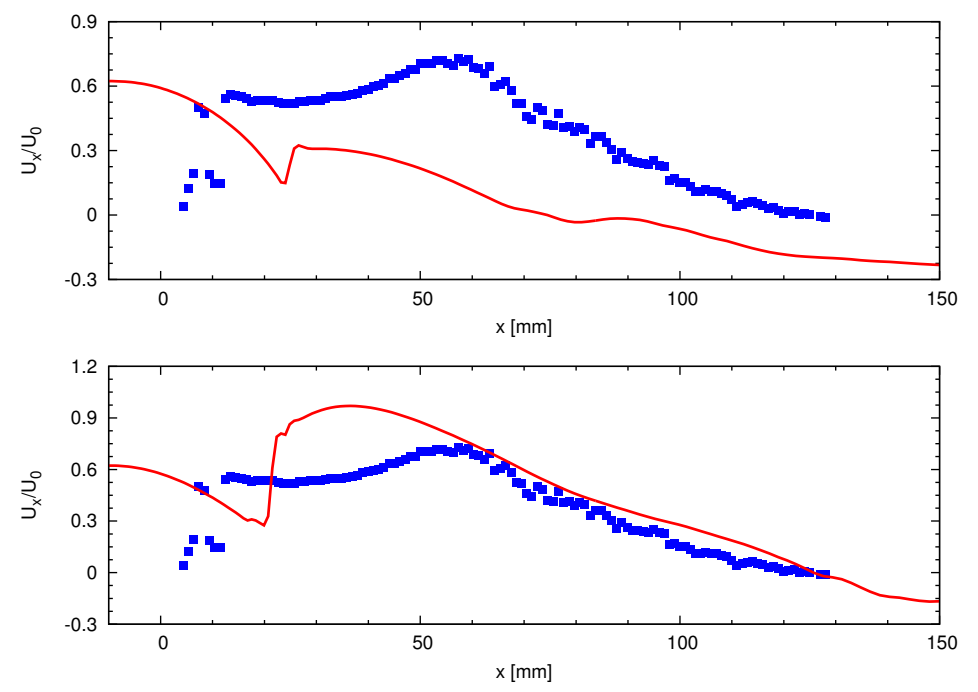

Figure 42: Comparison of mean axial velocity for the hydrogen/air flame with experiments (symbols) along the centerline. For the plot at the bottom the laminar flame speed and the reaction rates for hydrogen and progress variable are increased by a factor of three in order to compensate an error in calculation of the laminar flame speed for lean hydrogen premixed flames. 

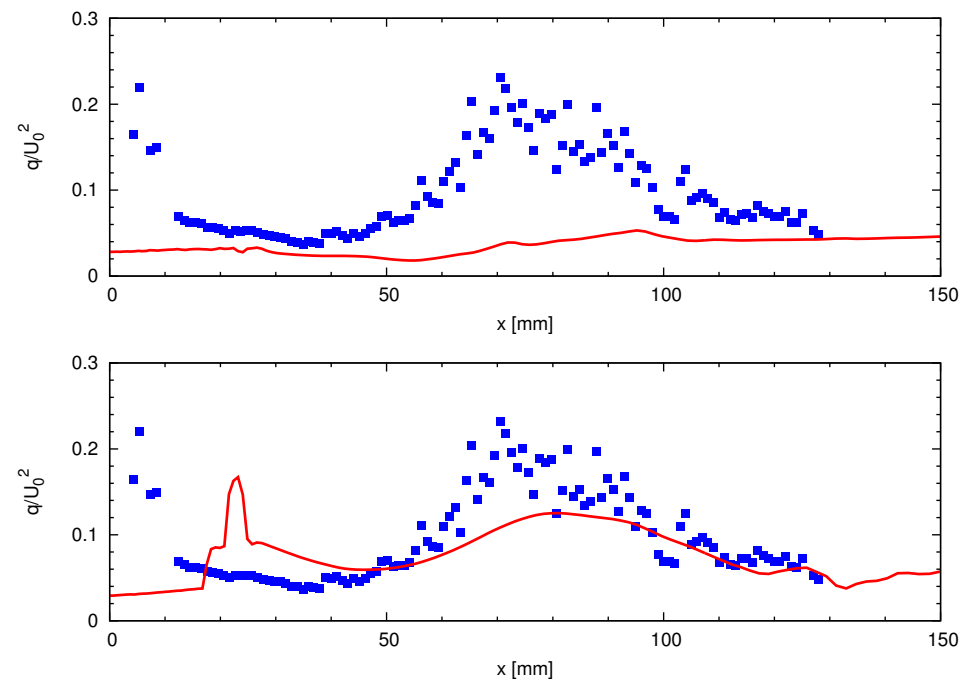

Figure 43: Comparison of 2D velocity fluctuations $\left(q=\sqrt{u_{x}^{\prime 2}+u_{r}^{\prime 2}}\right.$ - bottom) for the hydrogen/air flame with experiments (symbols) along the centerline. For the plot at the bottom the laminar flame speed is increased by a factor of three in order to investigate the effects of the laminar flame speed.
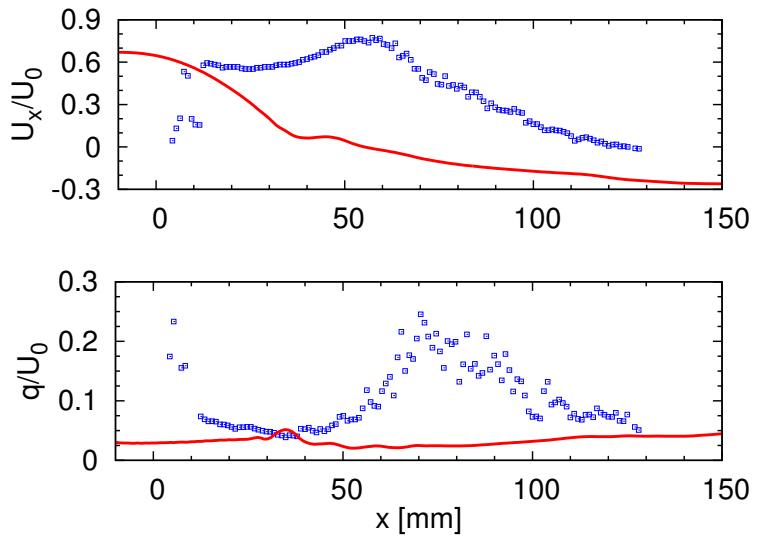

Figure 44: Comparison of mean axial velocity (top) and 2D velocity fluctuations $(q=$ $\sqrt{u_{x}^{\prime 2}+u_{r}^{\prime 2}}$ - bottom) for the hydrogen/air flame with experiments (symbols) along the centerline. LES results with unity Lewis number assumption are shown. 

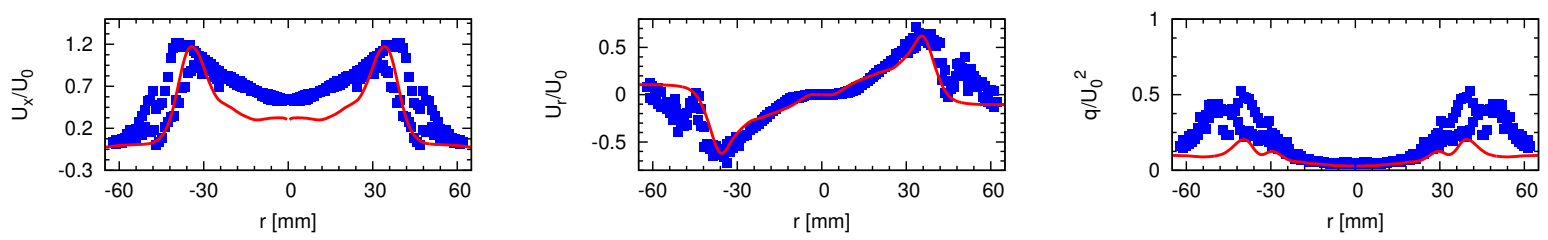

(a) $x=30 \mathrm{~mm}$
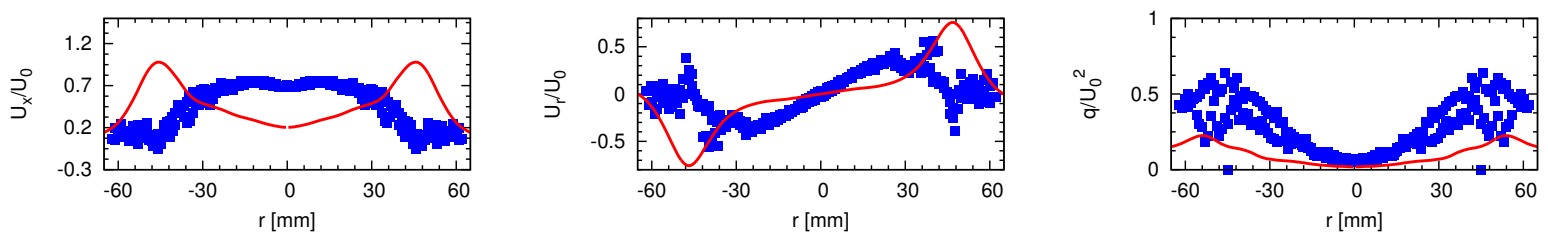

(b) $x=50 \mathrm{~mm}$
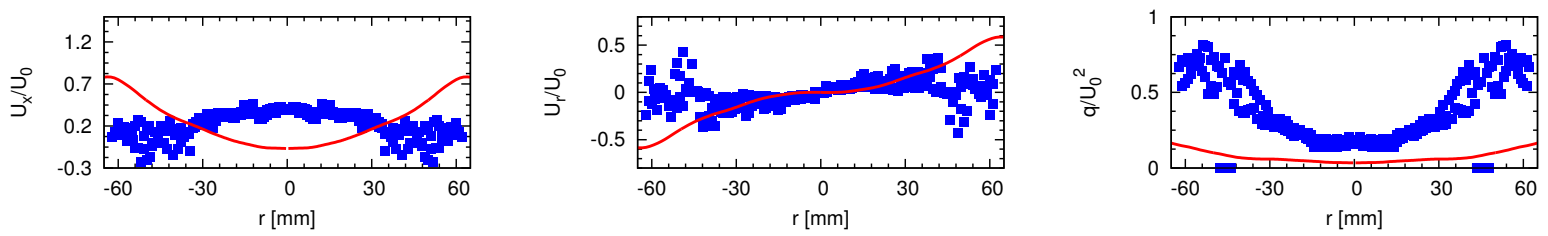

(c) $x=80 \mathrm{~mm}$

Figure 45: Comparison of mean axial velocity (left), mean radial velocity (center), and 2D velocity fluctuations $\left(q=\sqrt{u_{x}^{\prime 2}+u_{r}^{\prime 2}}\right.$ - right) for the hydrogen/air flame with experiments (symbols) at three locations downstream of the injection. 

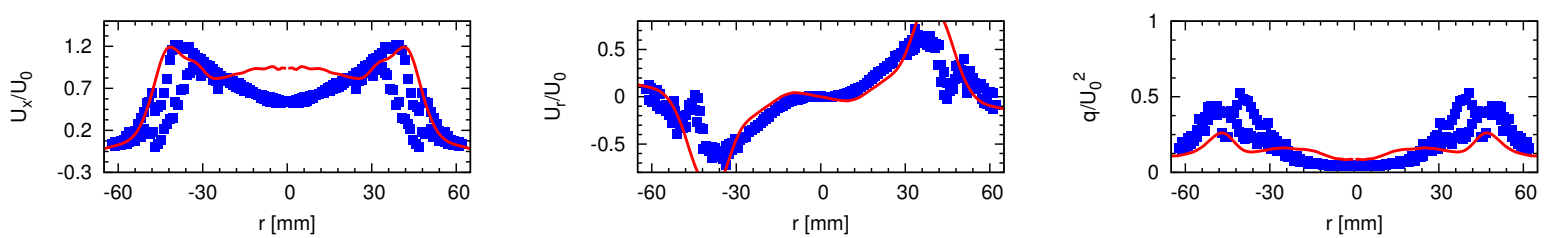

(a) $x=30 \mathrm{~mm}$
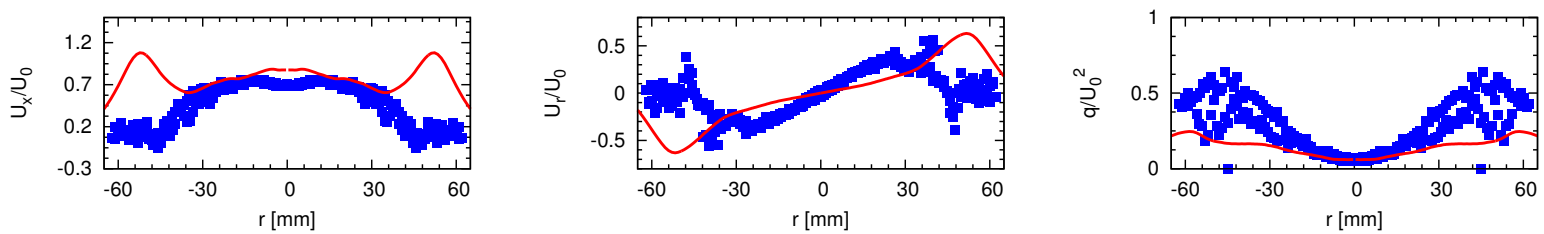

(b) $x=50 \mathrm{~mm}$
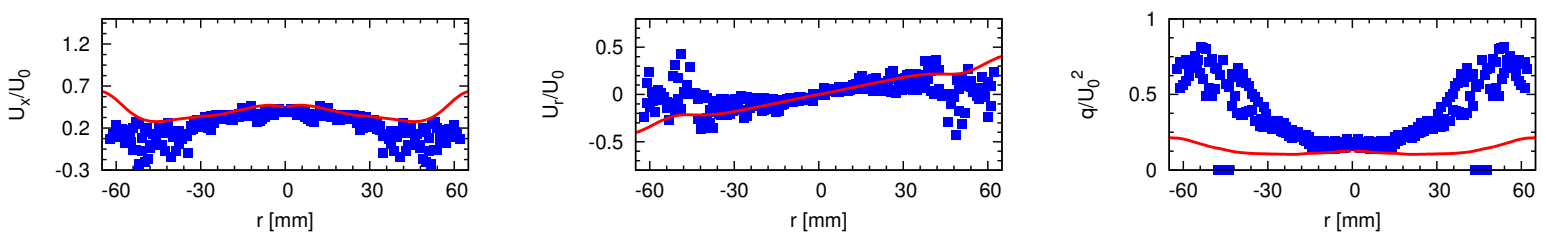

(c) $x=80 \mathrm{~mm}$

Figure 46: Comparison of mean axial velocity (left), mean radial velocity (center), and 2D velocity fluctuations $\left(q=\sqrt{u_{x}^{\prime 2}+u_{r}^{\prime 2}}\right.$ - right) for the hydrogen/air flame with experiments (symbols) at three locations downstream of the injection. The laminar flame speed is increased by a factor of three in order to investigate the effects of the laminar flame speed. 

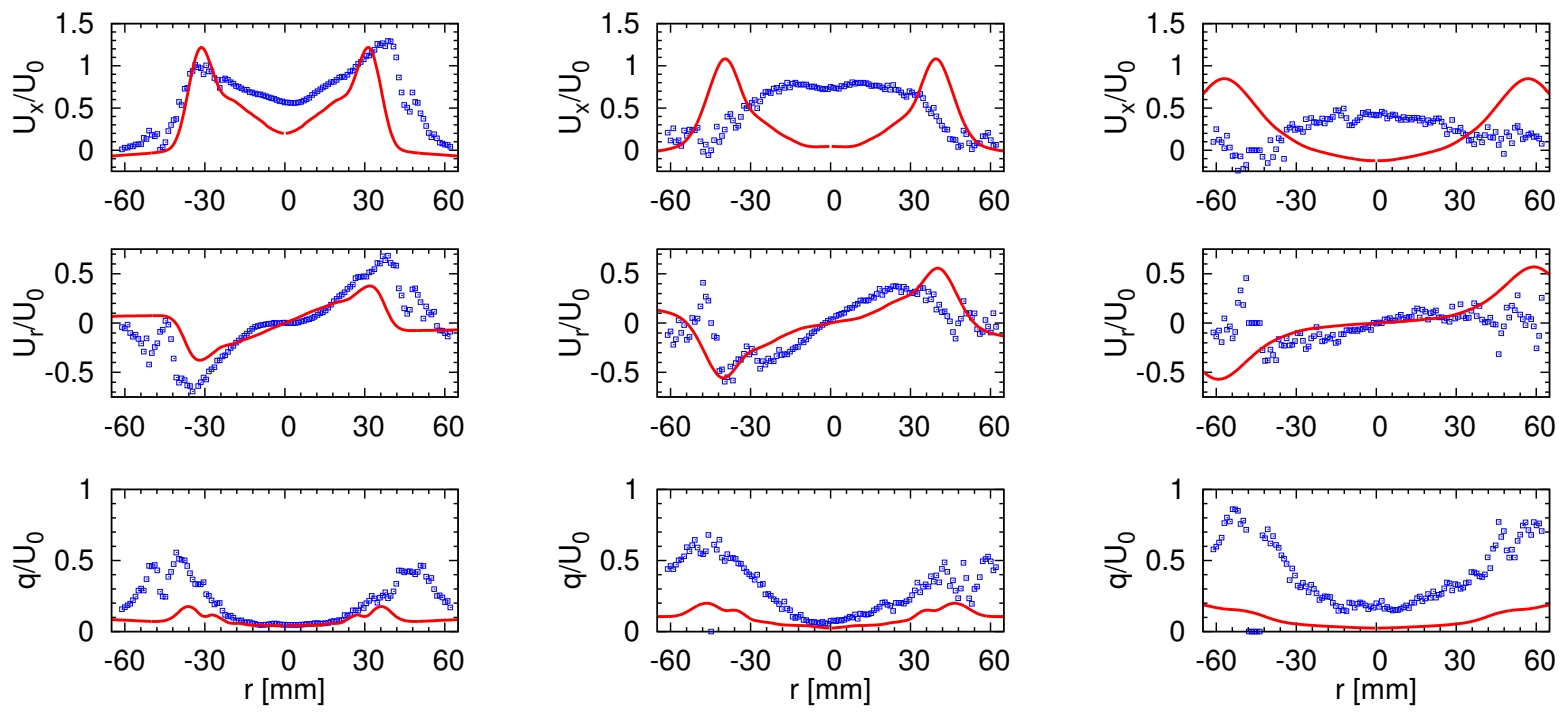

(a) $x=30 \mathrm{~mm}$

(b) $x=50 \mathrm{~mm}$

(c) $x=80 \mathrm{~mm}$

Figure 47: Comparison of mean axial velocity (top), mean radial velocity (center), and 2D velocity fluctuations $\left(q=\sqrt{u_{x}^{\prime 2}+u_{r}^{\prime 2}}\right.$ - bottom) for the hydrogen/air flame with experiments (symbols) at three locations downstream of the injection. LES results with unity Lewis number assumption are shown. 


\section{Bibliography}

[1] O. Desjardins, G. Blanquart, G. Balarac, and H. Pitsch. High order conservative finite difference scheme for variable density low mach number turbulent flows. J. Comp. Phys., $227(15): 7125-7159,2008$.

[2] E. Knudsen and H. Pitsch. A dynamic model for the turbulent burning velocity for large eddy simulation of premixed combustion. Comb. Flame, 154(4):740 - 760, 2008.

[3] E. Knudsen and H. Pitsch. A general flamelet transformation useful for distinguishing between premixed and non-premixed modes of combustion. Combustion and Flame, 156(3):678 - 696, 2009.

[4] H. Pitsch. Large-eddy simulation of turbulent combustion. Ann. Rev. Fluid Mech., 38:453-483, 2006.

[5] O. Colin, F. Ducros, D. Veynante, and T. Poinsot. A thickened flame model for large eddy simulations of turbulent premixed combustion. Phys. Fluids, 12(7):1843-1862, 2000 .

[6] H. Pitsch and L. Duchamp De Lageneste. Large-eddy simulation of premixed turbulent combustion using a level set approach. Proc. Comb. Inst., 29:2001-2008, 2002.

[7] H. Pitsch. A consistent level set formulation for large-eddy simulation of premixed turbulent combustion. Combust. Flame, 143:587-598, 2005.

[8] N. Peters. Turbulent Combustion. Cambridge University Press, England, 2000.

[9] M. Ihme. Pollutant Formation and Noise Emission in Turbulent Non-Premixed Flames. $\mathrm{PhD}$ thesis, Stanford University, 2007.

[10] K.-J. Nogenmyr, P. Petersson, X. S. Bai, A. Nauert, J. Olofsson, C. Brackman, H. Seyfried, J. Zetterberg, Z. S. Li, M. Richter, A. Dreizler, M. Linne, and M. Aldén. Large eddy simulation and experiments of stratified lean premixed methane/air turbulent flames. Proc. Comb. Inst., 31:1467-1475, 2006.

[11] C. Meneveau, T. S. Lund, and W. H. Cabot. A Lagrangian dynamic subgrid-scale model of turbulence. J. Fluid Mech., 319:353-385, 1996. 
[12] C. D. Pierce. Progress-Variable Approach for Large-Eddy Simulation of Turbulent Combustion. PhD thesis, Stanford University, 2001.

[13] V. Moureau, B. Fiorina, and H. Pitsch. A level set formulation for premixed combustion les considering the turbulent flame structure. Combustion and Flame, 156(4):801 - 812, 2009.

[14] C. Meneveau, T.S. Lund, and W.H. Cabot. A lagrangian dynamic subgrid-scale model of turbulence. J. Fluid Mech., 319:353-385, 2000.

[15] J. Reveillon and L. Vervisch. Response of the dynamic les model to heat release induced effects. Phys. Fluids, 8:2248-2250, 1996.

[16] B. Bédat and R. K. Cheng. Experimental study of premixed flames in intense isotropic turbulence. Combust. Flame, 100:485-494, 1995.

[17] R. K. Cheng. Velocity and scalar characteristics of premixed turbulent flames stabilized by weak swirl. Combust. Flame, 101:1-14, 1995.

[18] I. G. Shepherd and R. K. Cheng. The burning rate of premixed flames in moderate and intense turbulence. Combust. Flame, 127:2066-2075, 2001.

[19] P. Petersson, J. Olofsson, C. Brackman, H. Seyfried, J. Zetterberg, M. Richter, M. Aldén, M. A. Linne, R. K. Cheng, A. Nauert, D. Geyer, and A. Dreizler. Simultaneous PIV/OH-PLIF, rayleigh thermometry/OH-PLIF and stereo PIV measurements in a low-swirl flame. Applied Optics, 46(19):3928-3936, 2007.

[20] S. S. Girimaji and Y. Zhou. Analysis and modeling of subgrid scalar mixing using numerical data. Phys. Fluids, 8(5):1224-1236, 1996.

[21] J. U. Schluter, H. Pitsch, and P. Moin. Large eddy simulation inflow conditions for coupling with reynolds-averaged flow solvers. AIAA Journal, 42(3):478-484, 2004.

[22] R. K. Cheng and D. Littlejohn. Effects of combustor geometry on the flowfields and flame properties of a low-swirl injector. ASME - Proceedings of GT2008, 2008. 50504.

[23] R. K. Cheng, D. Littlejohn, P. A. Strakey, and T. Sidwell. Laboratory investigations of a low-swirl injector with $\mathrm{H}_{2}$ and $\mathrm{CH}_{4}$ at gas turbine conditions. Proc. Comb. Inst., pages 3001-3009, 2009.

[24] G. P. Smith, D. M. Golden, M. Frenklach, N. W. Moriarty, B. Eiteneer, M. Goldenberg, C. T. Bowman, R. K. Hanson, S. Song, Jr. W. C. Gardiner, V. V. Lissianski, and Z. Qin. http://www.me.berkeley.edu/gri_mech/.

[25] Scott G. Davis, Ameya V. Joshi, Hai Wang, and Fokion Egolfopoulos. An optimized kinetic model of h2/co combustion. Proceedings of the Combustion Institute, 30(1):1283 - 1292, 2005. 
[26] R. K. Cheng, D. Littlejohn, P. A. Strakey, and T. Sidwell. Laboratory investigations of a low-swirl injector with $\mathrm{H}_{2}$ and $\mathrm{CH}_{4}$ at gas turbine conditions. Proc. Comb. Inst., 32:3001-3009, 2009.

[27] H. Pitsch. FlameMaster: A C++ computer program for 0D combustion and 1D laminar flame calculations. Available from http://www.stanford.edu/ hpitsch/., 1998.

[28] S. Kadowaki and H. Suzuki, H.and Kobayashi. The unstable behavior of cellular premixed flames induced by intrinsic instability. Proc. Combust. Inst., 30:169-176, 2004.

[29] J. Yuan, Y. Ju, and C. K. Law. Coupled hydrodynamic and diffusional-thermal instabilities in flame propagation at subunity lewis numbers. Phys. Fluids, 17, 2005. 074106.

[30] J. .B Bell, R. K. Cheng, M. Day, and I. Shepherd. Numerical simulation of aLewis number effects on lean premixed turbulent flames. Proc. Comb. Inst., 31:1309-1317, 2007. 